\title{
WestVirginiaUniversity
}

THE RESEARCH REPOSITORY @ WVU

Graduate Theses, Dissertations, and Problem Reports

2012

\section{Oxide Thermoelectric Materials for High Temperature Power Generation}

Xueqin Chen

West Virginia University

Follow this and additional works at: https://researchrepository.wvu.edu/etd

\section{Recommended Citation}

Chen, Xueqin, "Oxide Thermoelectric Materials for High Temperature Power Generation" (2012). Graduate Theses, Dissertations, and Problem Reports. 4839.

https://researchrepository.wvu.edu/etd/4839

This Thesis is protected by copyright and/or related rights. It has been brought to you by the The Research Repository @ WVU with permission from the rights-holder(s). You are free to use this Thesis in any way that is permitted by the copyright and related rights legislation that applies to your use. For other uses you must obtain permission from the rights-holder(s) directly, unless additional rights are indicated by a Creative Commons license in the record and/ or on the work itself. This Thesis has been accepted for inclusion in WVU Graduate Theses, Dissertations, and Problem Reports collection by an authorized administrator of The Research Repository @ WVU. For more information, please contact researchrepository@mail.wvu.edu. 


\title{
Oxide Thermoelectric Materials for High Temperature Power Generation
}

\author{
Xueqin Chen
}

Thesis submitted to

the Benjamin M. Statler College of Engineering and Mineral Resources at West Virginia University

in partial fulfillment of the requirements for the degree of

\author{
Master of Science \\ in Mechanical Engineering \\ Xueyan Song, Ph.D., Chair \\ Ever J. Barbero, Ph.D. \\ Daneesh Simien, Ph.D. \\ Department of Mechanical Engineering
}

Morgantown, West Virginia

2012

Keywords: Thermoelectric (TE) materials, $\mathrm{Ca}_{3} \mathrm{Co}_{4} \mathrm{O}_{9}$, Seebeck Coefficiency, Electrical resistivity, Thermal conductivity, Power factor, Figure-of-merit 


\title{
ABSTRACT \\ Complex Thermoelectric Oxide for High Temperature Power Generation
}

\author{
Xueqin Chen
}

Power plants, turbine engines, automobiles and air conditioning, among others, release enormous amounts of heat into the environment, thereby wasting vast amounts of thermal energy. The typical U.S. power plant is only about 33\% efficient, using three units of fuel to produce one unit of electricity. The rest is released as waste heat into the atmosphere. In standard dieselpowered vehicles, up to $65 \%$ of the fuel is lost as waste heat, which is rejected through the cooling and exhaust systems at temperatures up to $900^{\circ} \mathrm{C}$. A potential way to improve the sustainability of our energy infrastructure and electricity base is through waste heat recovery using solid state thermoelectric power generators, which can directly convert temperature differentials into electrical power. Oxide materials, such as newly-developed nontoxic $\mathrm{Ca}_{3} \mathrm{Co}_{4} \mathrm{O}_{9}$, are particularly promising for thermoelectric applications because of their relatively high stability, even at high temperatures in air. The main challenge for enabling oxide thermoelectric materials is to improve their conversion efficiency, which is currently lower than that of conventional, low temperature thermoelectric materials. The conversion efficiency is usually evaluated by the dimensionless figure-of-merit $Z T=S^{2} T / \rho \kappa$, where $S, T, \rho$ and $\kappa$ are Seebeck coefficient (or thermoelectric power), absolute temperature, electrical resistivity, and thermal conductivity, respectively.

The main purpose of this project is to optimize the synthesis condition for powder precursor and pelletizing condition for bulk ceramic, to obtain the pure $\mathrm{Ca}_{3} \mathrm{Co}_{4} \mathrm{O}_{9}$ with high energy conversion efficiency. In the meanwhile, for practical applications, it is essential to understand the thermal stability of sintered $\mathrm{Ca}_{3} \mathrm{Co}_{4} \mathrm{O}_{9}$ materials in air, the formation of other cobaltite CaCo-O phases, and their property and structure evolution upon long term high temperature annealing and over-heating above the reported $\mathrm{Ca}_{3} \mathrm{Co}_{4} \mathrm{O}_{9}$ decomposition temperature of $926^{\circ} \mathrm{C}$, as indicated in the $\mathrm{CaO}-\mathrm{CoO}$ phase diagram. In details, the following is studied in this thesis: (1) Effect of calcinations temperature for the precursor powders on the thermoelectric properties of $\mathrm{Ca}_{3} \mathrm{Co}_{4} \mathrm{O}_{9}$; (2) Anisotropic thermoelectric properties in a misfit-layered calcium cobaltite affected by the conditions of pellets preparation. (3) Nanostructure and thermoelectric properties evolution of polycrystal $\mathrm{Ca}_{3} \mathrm{Co}_{4} \mathrm{O}_{9}$ upon over-heating condition.

The above study will be instrumental for fabricating the polycrystal $\mathrm{Ca}_{3} \mathrm{Co}_{4} \mathrm{O}_{9}$ with optimized microstructure and enhanced thermoelectric properties. Upon the completion of the microstructure and properties optimization of pure polycrystal $\mathrm{Ca}_{3} \mathrm{Co}_{4} \mathrm{O}_{9}$, investigation will be carried out to further improve the thermoelectric performance of $\mathrm{Ca}_{3} \mathrm{Co}_{4} \mathrm{O}_{9}$ through doping impurity and engineering the nanostructure.

Keywords: Thermoelectric (TE) Materials, $\mathrm{Ca}_{3} \mathrm{Co}_{4} \mathrm{O}_{9}$, Seebeck Coefficienct, Electrical Resistivity, Thermal Conductivity, Power Factor, Figure-of-merit 


\section{ACKNOWLEGEMENTS}

I would like to dedicate this thesis to my family including my parents, grandparents, my brother and sister, my dear husband and my lovely daughters, for their supports during my study.

I am sincerely grateful to my advisor Dr. Xueyan Song for her mentoring, encouragement, and financial support throughout this project. Her knowledge and hard work gave me inspiration and her persistence is my motivation during the course of this work.

I would like to thank the other two committee members, Dr. Ever J. Barbero and Dr. Daneesh Simien who split the time from their very busy schedules to assist me.

I would like to lay emphasis on thanks to Song Chen for his valuable assistance for TEM observations and thermoelectric properties measurements.

I would also like to thank one undergraduate student of WVU, Clinton P. Smith and a high school teacher, Eric W. Goff. Both of them worked with me last summer and gave me very helpful support.

I would also like to thank all the members in our research group. A great deal of thanks I would like to give Dr. Yun Chen and Dr. Xuezhang Xiao for all of their help. I would like to thank our other two group members, Diego Palacio and Maria Torres. It is very helpful to learn the courses and do some researches with them.

Special thanks to my dear friend Yi Yang for his assistance in E-beam lithography, E-Beam Evaporator and White Light Ellipsometer operations. Finally, I cannot express enough love and appreciation for my parents, grandparents, my brother and sister, my dear husband and my lovely daughters for all their supports. Everything I have achieved is because of them. 


\section{Table of Contents}

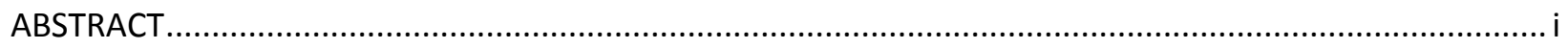

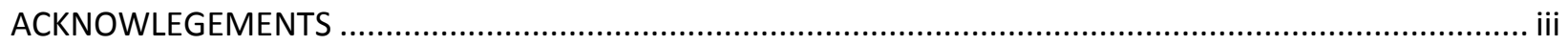

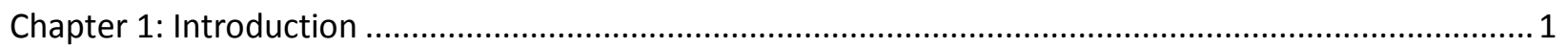

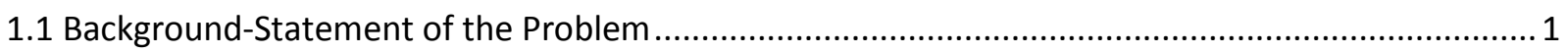

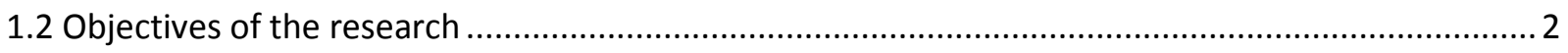

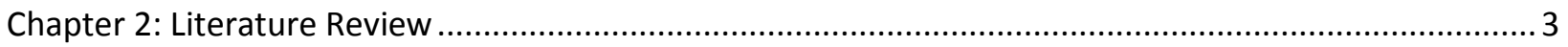

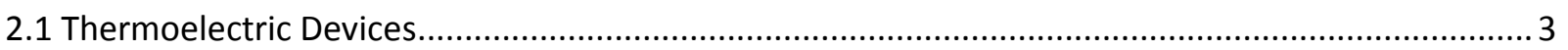

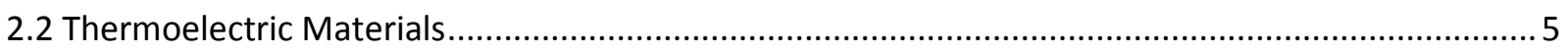

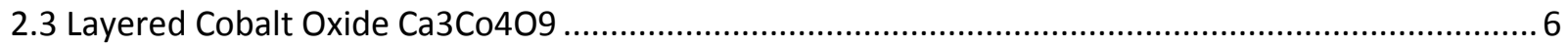

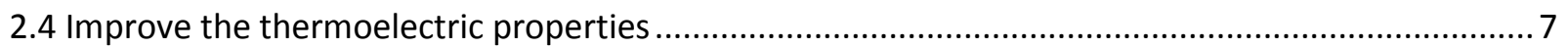

2.4.1 Optimize the TE performance by metal ion doping ........................................................... 7

2.4.2 Optimize the TE performance by special fabrication methods ............................................. 9

2.4.3 Optimize the TE performance by Minimizing the Thermal Conductivity ................................. 10

2.4.4 Control the particle size and grain alignment through preparation methods...........................11

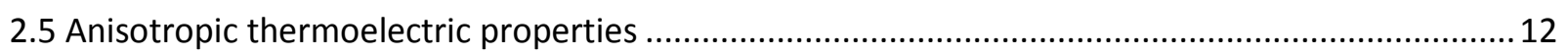

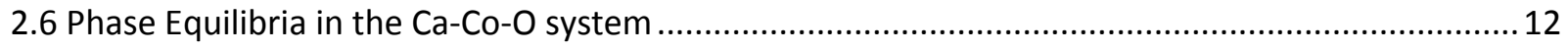

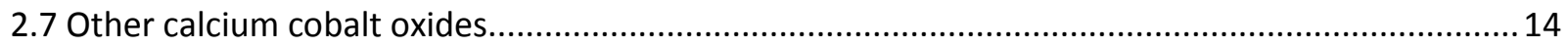

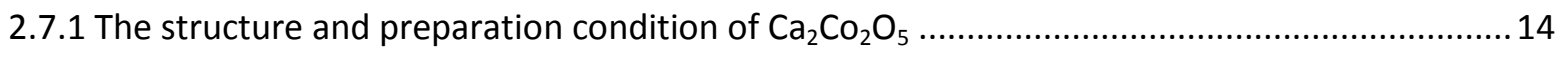

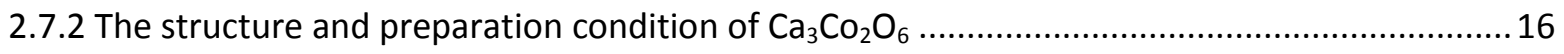

Chapter 3: Effect of Calcinations Temperature for Powder Precursor on the Thermoelectric Properties of

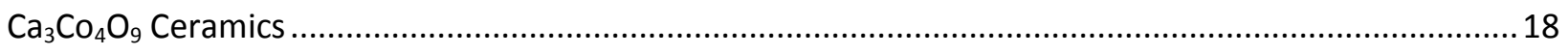

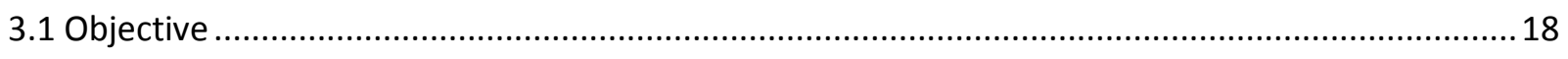

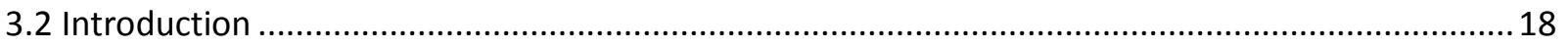

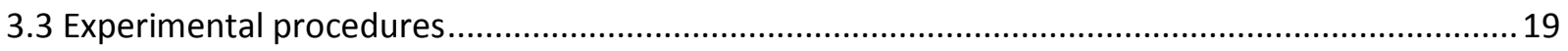

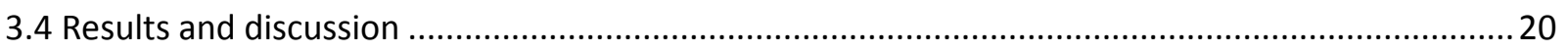

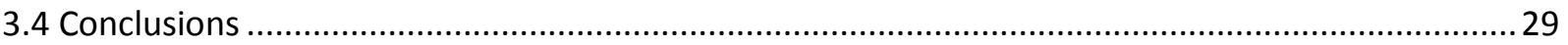

Chapter 4: Anisotropic Thermoelectric Properties in a Misfit-layered Calcium Cobaltite Affected by

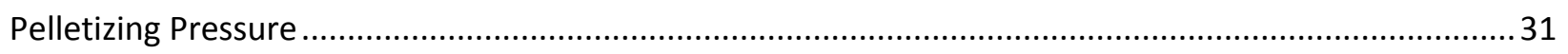

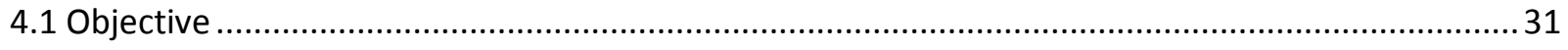

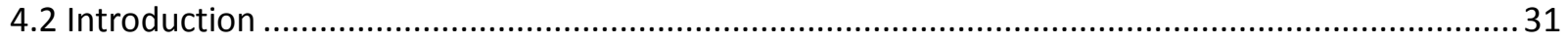




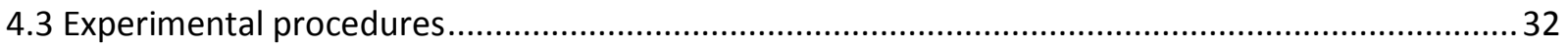

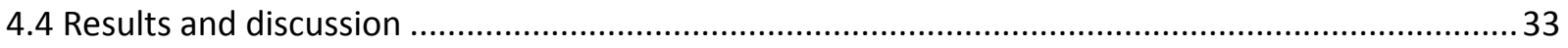

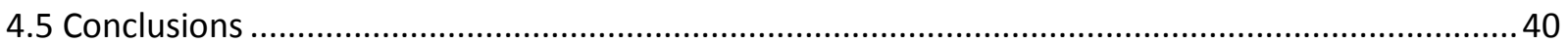

Chapter 5: $\mathrm{Ca}_{3} \mathrm{Co}_{4} \mathrm{O}_{9}$ Bulk Ceramics and Their Thermoelectric Properties and Nanostructure Evolution

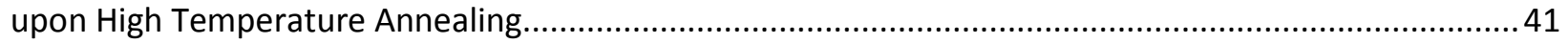

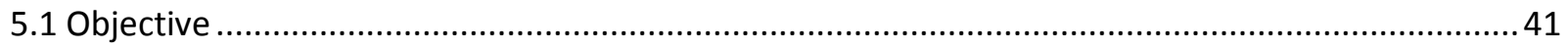

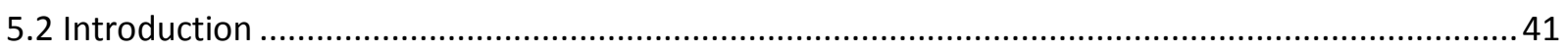

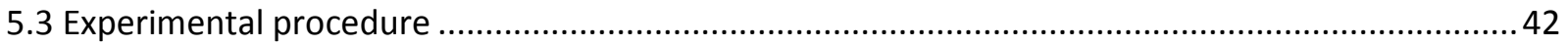

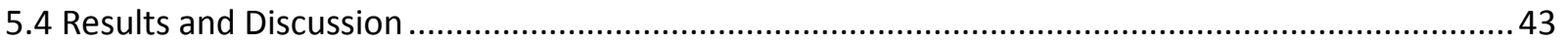

5.4.1 Thermoelectric performance of the baseline and annealed samples ..................................... 44

5.4.2 Microstructure Characterization using XRD and SEM ......................................................... 45

5.4.3 Nanostructure and Chemistry Analysis Using TEM............................................................. 47

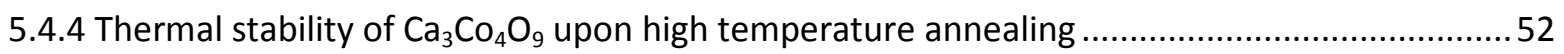

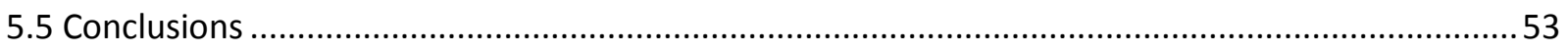

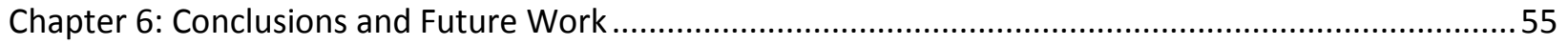

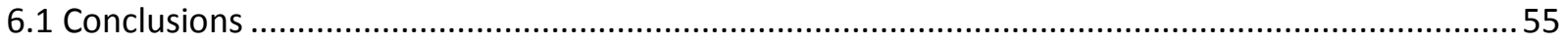

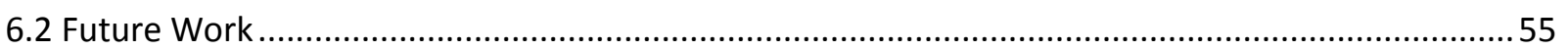

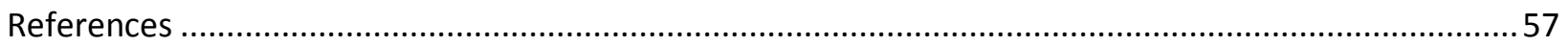




\section{LIST OF FIGURES}

Figure 2. 1 TE heat engines. (A) when heat flows across the junction, electrical current is generated through the Seebeck effect. (B) when current runs across a TE junction, it heats or cools through the Peltier effect, depending on the direction of the current flow. ${ }^{[10]}$

Figure 2. 2 Proposed structural model for " $\mathrm{Ca}_{3} \mathrm{Co}_{4} \mathrm{O}_{9}$ " involving a supercell with $\mathrm{b} \sim 8 \mathrm{~b}_{1} \sim 13 \mathrm{~b}_{2}{ }^{[13]}{ }^{[1 . \ldots . . .6} 6$ Figure 2. 3 Electrical conductivity (a), Seebeck coefficient (b), thermal conductivity (c) and figure of merit (ZT) (d) for Co349. (The figure is cited from a review, the number 31-50 in the figure refers the reference number in this review. $)^{[15]}$

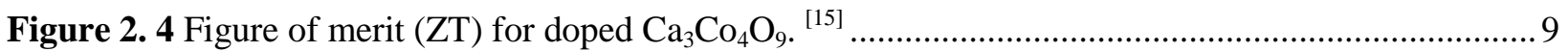

Figure 2. 5 SEM micrographs of fractured surfaces of the $\mathrm{Ca}_{3} \mathrm{Co}_{4} \mathrm{O}_{9}$ samples processed by: ${ }^{[4,16]}$ (a) conventional sintering (CS), (b) hot-pressing (HP), (c) spark plasma sintering (SPS) processing and (d) cold high-pressure fabrication.

Figure 2. 6 Phase equilibrium diagram for the system Ca-Co-O system in air. ${ }^{[19]}$.................................. 13

Figure 2. 7 (a) XRD patterns of $\mathrm{Ca}_{2-\mathrm{x}} \mathrm{Bi}_{\mathrm{x}} \mathrm{Co}_{2} \mathrm{O}_{5}$ samples. The insert shows SEM picture of $\mathrm{Ca}_{1.98} \mathrm{Bi}_{0.02} \mathrm{Co}_{2} \mathrm{O}_{5}$ sample, ${ }^{[23]}$ (b) Typical XRPD pattern of a calcium cobaltite thin film (Ca/Co = 1) grown on NdGaO3 under optimized conditions. The peak labeled with a star is generated by the sample holder. ${ }^{[25]}$

Figure 2. 8 (a) Projection onto (001) of the $\mathrm{Ca}_{3} \mathrm{Co}_{2} \mathrm{O}_{6}$ structure showing the triangular arrangement of the chains, (b) Co206 0 chain evidencing the alternation of cobalt in octahedral and prismatic coordination. [27]

Figure 3. 1 X-ray diffraction pattern of Co349 pellet made by powder which was calcined at $650^{\circ} \mathrm{C}$ ( the pressed pellet was sintered at $920^{\circ} \mathrm{C}$ for $\left.12 \mathrm{~h}\right)$.

Figure 3. 2 SEM images (scale bar represents $1 \mu \mathrm{m}$ ) for Co349 powder calcined at different temperature: $650^{\circ} \mathrm{C}, 700^{\circ} \mathrm{C}, 750^{\circ} \mathrm{C}$, and $800^{\circ} \mathrm{C}$.

Figure 3. 3 SEM images (scale bar represents $100 \mathrm{~nm}$ ) for Co349 powder calcined at different temperature: (A) $650^{\circ} \mathrm{C}$, (B) $700^{\circ} \mathrm{C}$, (C) $750^{\circ} \mathrm{C}$, and (D) $800^{\circ} \mathrm{C}$.

Figure 3. 4 SEM micrographs measured on pressed surface corresponding to those sintered bodies: (A) Co349-650, (B) Co349-700, (C) Co349-750, and (D) Co349-800, respectively. All the pellets were annealed at $920{ }^{\circ} \mathrm{C}$ for $12 \mathrm{hrs}$.

Figure 3. 5 A comparison of SEM micrographs (scale bar: $10 \mu \mathrm{m}$ ) measured on fractured surface corresponding to those sintered samples: Co349-650, Co349-700, Co349-750, and Co349-800. All the pellets were annealed at $920{ }^{\circ} \mathrm{C}$ for $12 \mathrm{hrs}$.

Figure 3. 6 Temperature dependence of the (a) electrical resistivity $\rho$, (b) Seebeck coefficient $S$, and (c) Power factor $S^{2} / \rho$ of Co349-650, Co349-700, Co349-750 and Co349-800.

Figure 3. 7 Temperature dependence of the thermal conductivity of Co349-650, Co349-700, Co349-750 and Co349-800 along the out-of-plane (pressing) direction.

Figure 3. 8 Temperature dependence of $\kappa_{\text {car }}$ and $\kappa_{\text {ph }}$ for the samples prepared with the powder precursor 
Figure 4. 1 SEM surface morphology of Co349 sintered at $920^{\circ} \mathrm{C}$ for $24 \mathrm{~h}$ with different pelletizing condition: (a) 0.25GPa (Co349-0.25), (b) 0.5GPa (Co349-0.5), and (c) 1.0GPa (Co349-1.0) 34

Figure 4. 2 SEM fractured surface morphology of Co349 sintered at $920^{\circ} \mathrm{C}$ for $24 \mathrm{~h}$ with different pelletizing condition: (a) 0.25GPa (Co349-0.25), (b) 0.5GPa (Co349-0.5), and (c) 1.0GPa (Co349-1.0). 35 Figure 4. $3 \mathrm{X}$-ray diffraction pattern of $\mathrm{Ca}_{3} \mathrm{Co}_{4} \mathrm{O}_{9}$ pellet pressed under different pressure and powder baseline (the pressed pellet and baseline sample was sintered at $920^{\circ} \mathrm{C}$ for $24 \mathrm{~h}$ )... 36

Figure 4. 4 Temperature dependence of the (a) Seebeck coefficient $S$, (b) electrical resistivity $\rho$, and (c) Power factor $S^{2} / \rho$ of Co349-1.0GPa, Co349-0.5GPa and Co349-0.25GPa. 38

Figure 4. 5 Temperature dependence of the (a) thermal conductivity, (b) ZT of Co349-1GPa, Co3490.5GPa and Co349-0.25GPa. Empty symbols represent the value across in-plane; filled symbols represent the values along in-plane. 39

Figure 5. 1 Temperature dependence of the (a) Seebeck coefficient $S$, (b) electrical resistivity $\rho$, and (c) Power factor $S^{2} / \rho$ of Co349-baseline and Co349-annealed..

Figure 5. 2 Temperature dependence of the thermal conductivity $\kappa$. Empty symbols represent the value along out-of-plane; filled symbols represent the values along in-plane... 44

Figure 5. 3 Temperature dependence of ZT for Co349-Baseline and Co349-Annealed samples.............. 45

Figure 5. 4 XRD patterns for Co349-Baseline and Co349-Annealed samples....................................... 46

Figure 5. 5 SEM images for Co349-Baseline (a) and Co349-Annealed (b) samples................................ 46

Figure 5. 6a Baseline sample: Crystal bundles with different orientation. b-d Baseline sample: Electron diffraction taken from different nano-lamella (b,c) and the magnified view of nano-lamella within one bundle. 48

Figure 5. 7 Baseline sample: HRTEM images showing the nano-lamella with the same orientation along the $c$ axis and different orientation along the $a b$ plane.

Figure 5. 8 Annealed sample: Nano-lamella Co349 and their electron diffraction pattern......................50

Figure 5. 9 Annealed sample: Co-containing $\mathrm{CaO}$ in between the Co349 nano-lamella. (c) is indexing for (b). 51

Figure 5. 10 Annealed sample: Nano-lamella Co349 (CCO349) and Co225 (CCO225). .51

Figure 5. 11 Annealed sample: Diffraction pattern from Co225 (a) and the interface between Co225 and Co349 (b) 52

Figure 5. 12 Annealed sample: Nano-lamella Co349 and Co225.. .53 


\section{LIST OF TABLES}

Table 4. 1 Lotgering factors for Samples pelletizing under different pressure

Table 5. 1 EDS result of $\mathrm{CaO}$ from annealed sample.

Table 5. 2 EDS of Co225..... 


\section{Chapter 1: Introduction}

\subsection{Background-Statement of the Problem}

Today energy and the environment have become some of the most critical current issues. The need for sources of energy other than fossil fuels, as well as the most efficient use of our current fossil fuel supply, has sparked significant research into alternative energy sources and different types of energy conversion technologies. One of the types of energy conversion technologies that have received renewed attention is thermoelectric energy conversion, where heat is converted directly into electricity using a class of materials known as thermoelectric (TE) materials.

The energy conversion efficiency of a TE material is usually evaluated by the dimensionless figure-of-merit

$$
Z T=S^{2} T / \rho \kappa
$$

where $S, T, \rho$ and $\kappa$ are Seebeck coefficient (or thermoelectric power), absolute temperature, electrical resistivity, and thermal conductivity, respectively. For practical use, $Z T$ of a thermoelectric material should be at least greater than 1 . The thermal conductivity $\kappa$ is related to the transfer of heat through a material, either by the carriers (electrons or holes) or by quantized vibrations of the lattice, called phonons, which can be represented by $\kappa_{\text {car }}$ and $\kappa_{\mathrm{ph}}$ respectively ( $\kappa$ $\left.=\kappa_{\mathrm{car}}+\kappa_{\mathrm{ph}}\right)$. According to the Wiedemann-Franz law, the electronic term $\kappa_{\mathrm{car}}$ is directly related to the electrical resistivity represented by the following relationship:

$$
\kappa_{\mathrm{car}}=L T / \rho=n e \mu L T
$$

where $L$ is the Lorenz factor, $2.4 \times 10^{-8} \mathrm{~J}^{2} \mathrm{~K}^{-2} \mathrm{C}^{-2}$ for free electrons. From equation 1.2 , it is clearly seen that a lower electrical resistivity will be accompanied by a higher thermal conductivity $\kappa_{\text {car }}$ contributed by electron or hole transportation, i.e., the product of $\rho$ and $\kappa_{\text {car }}$ is essentially constant at a given temperature.

Compared with those conventional thermoelectric materials such as metal chalcogenides, transition metal disilicates, and silicon-germanium alloys, layered metal oxides have attracted much attention since the discovery of high TE performance in $\gamma-\mathrm{Na}_{x} \mathrm{CoO}_{4}$ by Terasaki et al... ${ }^{[1]}$ However, due to the volatility and poor resistance to humidity, practical application of $\mathrm{NaCo}_{2} \mathrm{O}_{4}$ for power generation is quite limited. Another cobaltite $\mathrm{Ca}_{3} \mathrm{Co}_{4} \mathrm{O}_{9}$ was considered as most promising candidate for a p-type high temperature TE material due to its stability and low toxicity at high temperature. The crystal structure of $\mathrm{Ca}_{3} \mathrm{Co}_{4} \mathrm{O}_{9}$ is a misfit-layered structure consisting of an electrically conductive $\mathrm{CdI}_{2}$-type $\mathrm{CoO}_{2}$ layer and an insulating $\mathrm{Ca}_{2} \mathrm{CoO}_{3}$ rocksalt-type layer, with the extrapolated $Z T=0.83$ at $973 \mathrm{~K}$ for the single crystal. ${ }^{[2]}$ However the $Z T$ of the polycrystalline bulk $\mathrm{Ca}_{3} \mathrm{Co}_{4} \mathrm{O}_{9}$ is still at a relatively low level of 0.1-0.4. Highefficiency thermoelectric conversion requires simultaneously low electrical resistivity and low thermal conductivity. To optimize the TE performance of $\mathrm{Ca}_{3} \mathrm{Co}_{4} \mathrm{O}_{9}$ ceramics, some special 
fabrication methods for bulk materials were chosen ${ }^{[3-7]}$, such as Hot-Pressing (HP), Cold high pressure pressing, Magnetic Alignment (MA) and Spark Plasma Sintering (SPS) process. However, according to the Wiedemann-Franz Law, the highly textured polycrystalline samples usually have high thermal conductivities. As a result the fabrication of fine microstructure in a sintered body of $\mathrm{Ca}_{3} \mathrm{Co}_{4} \mathrm{O}_{9}$ is the only remaining choice for reduction of phonon contribution to thermal conductivity and enhancing the thermoelectric conversion efficiency. Significant engineering of nanostructure is required to enhance the TE properties of polycrystal $\mathrm{Ca}_{3} \mathrm{Co}_{4} \mathrm{O}_{9}$.

\subsection{Objectives of the research}

This research is aimed at optimizing the materials synthesis and pellets fabrication conditions, to enhance the TE properties of the baseline sample, i.e. pure polycrystal $\mathrm{Ca}_{3} \mathrm{Co}_{4} \mathrm{O}_{9}$. The present work reports on the thermoelectric performance and nanostructure of polycrystalline $\mathrm{Ca}_{3} \mathrm{Co}_{4} \mathrm{O}_{9}$ samples, synthesized using the optimized sol-gel route, which is with low cost and easy stoichiometry control. In particular, we studied the following:

- Effect of calcinations temperature for the powder precursor on the TE properties of $\mathrm{Ca}_{3} \mathrm{Co}_{4} \mathrm{O}_{9}$

- Anisotropic TE properties in a misfit-layered calcium cobaltite affected by pelletizing pressure

- Nanostructure and TE properties evolution of polycrystal $\mathrm{Ca}_{3} \mathrm{Co}_{4} \mathrm{O}_{9}$ upon over-heating condition.

The main purpose of this project is to optimize the calcinations condition for powder precursor and pelletizing condition for bulk material to obtain the highest figure-of-merit of $\mathrm{Ca}_{3} \mathrm{Co}_{4} \mathrm{O}_{9}$ ceramic materials. In the meanwhile, for practical applications, it is essential to understand the thermal stabilities of sintered $\mathrm{Ca}_{3} \mathrm{Co}_{4} \mathrm{O}_{9}$ materials in air, the formation of other cobaltite Ca-Co$\mathrm{O}$ phases, and their property and structure evolution upon long term high temperature annealing and over-heating above the reported decomposition temperature of $926^{\circ} \mathrm{C}$, as indicated in the $\mathrm{CaO}-\mathrm{CoO}$ phase diagram.

The above study will be instrumental for fabricating the pure polycrystal $\mathrm{Ca}_{3} \mathrm{Co}_{4} \mathrm{O}_{9}$ with optimized microstructure and TE properties. Upon the completion of the structure and properties optimization of pure polycrystal $\mathrm{Ca}_{3} \mathrm{Co}_{4} \mathrm{O}_{9}$, further investigation will be carried out to study the effect of impurity doping and nano-inclusion on the TE performance. 


\section{Chapter 2: Literature Review}

\subsection{Thermoelectric Devices}

Thermoelectric (TE) material is a material that can convert heat into electricity directly through one of the most fundamental TE phenomena, the Seebeck effect. The Seebeck coefficient, or thermopower, is a measure of the magnitude of an induced thermoelectric voltage in response to a temperature difference across the material. The Seebeck effect and two related phenomena (the Peltier effect and Thomson effect) are together called the "thermoelectric effect". In the early 1800s, Seebeck observed that when two different materials are joined together and the junctions are held with a temperature difference $(T)$, a voltage difference $(\Delta V)$ proportional to the temperature gradient $(\Delta T)$ will be developed. The ratio of the developed voltage to the temperature gradient $(\Delta V / \Delta T)$ is related to an intrinsic property of the materials called the Seebeck coefficient, $S$. The Seebeck coefficient is very low for metals (only a few $\mu V / K$ ) and much larger for semiconductor.

According to the equation 1.1, it is clearly seen that good TE materials should have large $S$ along with low electrical resistivity $\rho$ and thermal conductivity $\kappa$. Nevertheless, these three parameters are not independent; they all strongly depend on the crystalline structure, energy band, carrier concentration, etc., of a system. ${ }^{[8]}$ For instance, with the increase in carrier concentration, $\rho$ will decrease but simultaneously $S$ will also lessen.

The thermopower, or Seebeck coefficient, can be also thought of as the heat per carrier over temperature, more simply, the entropy per carrier,

$$
S \approx \frac{C}{q}
$$

where $C$ is the specific heat and $q$ is the charge of the carrier. ${ }^{[9]}$ For metals, the heat per carrier is essentially a product of the electronic specific heat and the temperature divided by the number of carriers (N), i.e.,

$$
S \approx \frac{C T}{N} \approx\left(\frac{k_{B}}{e}\right) \frac{k_{B} T}{E_{F}}
$$


where $k_{\mathrm{B}}$ is the Boltzmann constant, $E_{\mathrm{F}}$ is the Fermi energy. The quantity $k_{\mathrm{B}} / \mathrm{e} \approx 87 \mu \mathrm{V} / \mathrm{K}$ is a constant that represents the thermopower of a classical electron gas. Metals have thermopower values of much less than $87 \mu \mathrm{V} / \mathrm{K}$.

In semiconductor, a charged particle must first be excited across an energy gap $E_{\mathrm{g}}$. Thus, the thermopower is larger than the characteristic value of $87 \mu \mathrm{V} / \mathrm{K}$. Semiconductors can exhibit either electron conduction (negative thermopower) or hole conduction (positive thermopower). Typical thermopower values required for good TE performance are on the order of 150-250 $\mu \mathrm{V} / \mathrm{K}$ or greater.

For high-temperature applications, it is important to minimize the minority carriers in order to maintain a high thermopower. Again, for semiconductors, the carriers must be thermally excited across a gap for conduction to occur. There are two primary ways to achieve a high conductivity in semiconductor, either by minimizing the energy gap to excite across or by having very high mobility carriers.

The thermal conductivity $\kappa$ is related to the transfer of heat through a material, either by the carriers or by phonons, which is represented by $\kappa_{\text {car }}$ and $\kappa_{\mathrm{ph}}$ respectively $\left(\kappa=\kappa_{\mathrm{car}}+\kappa_{\mathrm{ph}}\right)$. The electrical conductivity and the thermal conductivity are tied through the Wiedemann-Franz law. Typical thermal conductivity values for a good TE material are less than $2 \mathrm{Wm}^{-1} \mathrm{~K}^{-1}$, and typically, $\kappa_{\mathrm{car}} \approx \kappa_{\mathrm{ph}}$.

The Peltier effect is the basis for many modern-day TE refrigeration devices, but the Seebeck effect is the basis for TE power-generation devices. The versatility of TE materials is illustrated in Fig. 2.1, ${ }^{[10]}$ which shows a TE couple composed of an $n$-type (negative thermopower and electron carriers) and p-type (positive thermopower and hole carriers) semiconductor material connected through metallic electrical contact pads. Both refrigeration and power generation may be accomplished using the same module, as shown in this figure. ${ }^{[10]}$ A TE module or device is built up of an array of these couples, arranged electrically in series and thermally in parallel. Thermoelectric energy conversion utilizes the Seebeck effect, wherein a temperature gradient is imposed across the device, resulting in a voltage that can be used to drive a current through a load resistance or device (as shown in Fig 2.1a). This is the direct conversion of heat into electricity. Conversely, the Peltier heat generated when an electric current is passed through a TE 
material provides a temperature gradient, with heat being absorbed on the cold side, transferred through (or pumped by) the TE materials, and rejected at the sink, thus providing a refrigeration capability (as shown in Fig 2.1b). The advantages of TE solid-state energy conversion are compactness, quietness (no moving parts), and localized heating or cooling. In addition, energy in the form of waste heat ( $0 \%$ efficiency) that would normally be lost may be converted into useful electrical energy (> 7-8\% efficiency) using a TE power-generation device. ${ }^{[11]}$
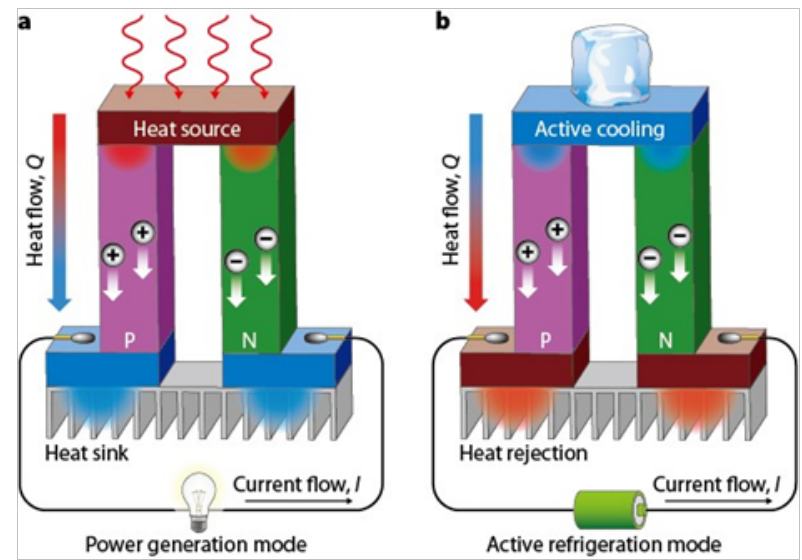

Figure 2. 1 TE heat engines. (A) when heat flows across the junction, electrical current is generated through the Seebeck effect. (B) when current runs across a TE junction, it heats or cools through the Peltier effect, depending on the direction of the current flow. ${ }^{[10]}$

\subsection{Thermoelectric Materials}

Thermoelectric (TE) materials are solid-state converters, which convert waste heat into electricity directly using the integration of the thermal, electrical, and semiconducting properties. These materials can be competitive with fluid-based systems, used in air-conditioning compressors or heat pumps, or used in smaller-scale applications such as in automobile seats, night-vision systems, and electrical-enclosure cooling.

Energy and the environment have become some of the most critical current issues. At the same time, waste heat from automobiles, factories, and similar sources offers a high-quality energy sources equal to about $70 \%$ of the total primary energy, but it is difficult to reclaim because its sources are small and widely dispersed. ${ }^{[12]}$ Consequently, the development of higherperformance TE materials is becoming more and more important. Proposed industrial and military applications of TE materials are generating increased activity in this field to reduce our 
dependence on fossil fuels. Power-generation applications are currently being investigated by the automotive industry as a means to develop electrical power from waste engine heat from the radiator and exhaust systems for use in next-generation vehicles.

\subsection{Layered Cobalt Oxide Ca3Co4O9}

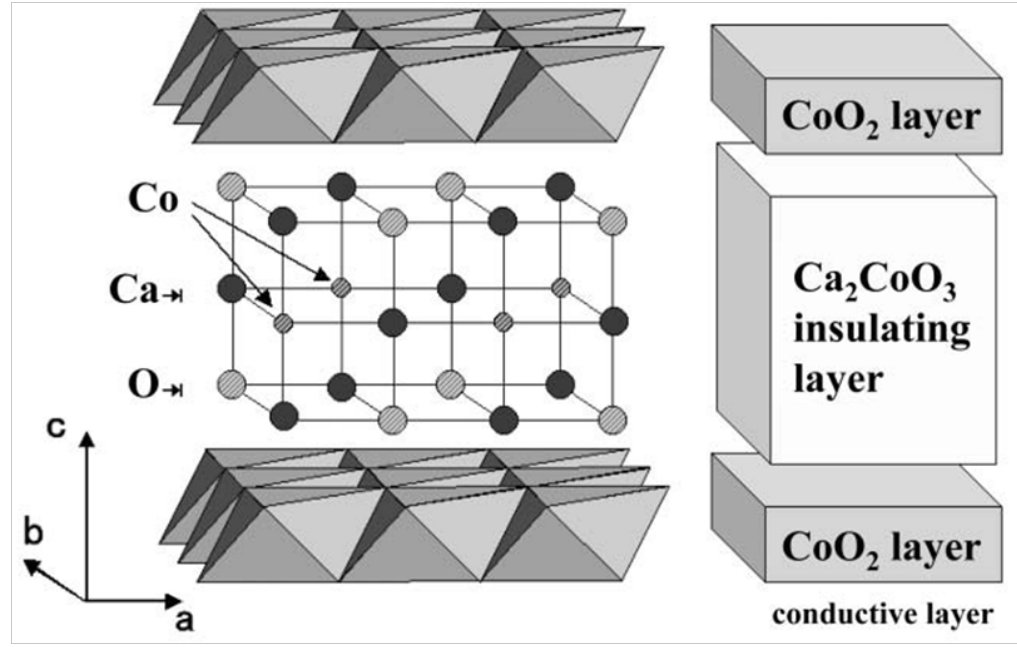

Figure 2. 2 Proposed structural model for “ $\mathrm{Ca}_{3} \mathrm{Co}_{4} \mathrm{O}_{9}$ ” involving a supercell with $\mathrm{b} \sim 8 \mathrm{~b}_{1} \sim 13 \mathrm{~b}_{2}$. ${ }^{[13]}$

Although conventional thermoelectric materials, such as the Bi-Te system, have a high ZT of around 1 near room temperature, their chemical and thermal stability is unsatisfactory for usage in converting waste heat at high temperature in air. Metal oxides were launched just after Terasaki et al. ${ }^{[1]}$ found a large thermpower $\left(100 \mu \mathrm{V} \mathrm{K}^{-1}\right.$ at $\left.300 \mathrm{~K}\right)$ coexisting with a low resistivity $(200 \mu \Omega \mathrm{cm}$ at $300 \mathrm{~K})$ in $\mathrm{NaCo}_{2} \mathrm{O}_{4}$ single crystal. Three years later, layered cobalt oxide $\mathrm{Ca}_{3} \mathrm{Co}_{4} \mathrm{O}_{9}$ was reported to possess fairly good TE performance, and unlike $\mathrm{NaCo}_{2} \mathrm{O}_{4}$, it resists oxidation in air at high temperature, is thermally and chemically stable and does not exhibit any polluting or toxic character. The structural model for $\mathrm{Ca}_{3} \mathrm{Co}_{4} \mathrm{O}_{9}$ is shown in Fig 2.2. ${ }^{[13]}$ This compound is a misfit-layered oxide consisting in two monoclinic subsystems with identical $a, c$, and $\beta$ parameters, but different $b$ parameters: $a=4.8376(7) \AA ̊, c=10.833(1) \AA$, and $\beta=$ $98.06(1)^{\circ}, \mathrm{b} 1=4.5565(6) \AA$, and $\mathrm{b} 2=2.8189(4) \AA$. The crystal structure is built up from the stacking along $c$ of triple rock salt-type layers $\mathrm{Ca}_{2} \mathrm{CoO}_{3}$ (first subsystem) with single $\mathrm{CdI}_{2}$-type $\mathrm{CoO}_{2}$ layers (second subsystem). 
For $\mathrm{Ca}_{3} \mathrm{Co}_{4} \mathrm{O}_{9}$ single crystals, a $\mathrm{ZT}$ value of 0.87 at $973 \mathrm{~K}$ in air was extrapolated. However, single crystal is hard to produce and also hard to be integrated in TE devices. Consequently, efforts have focused on $\mathrm{Ca}_{3} \mathrm{Co}_{4} \mathrm{O}_{9}$ polycrystalline materials to improve its figure of merit. To optimize the TE performance of $\mathrm{Ca}_{3} \mathrm{Co}_{4} \mathrm{O}_{9}$ ceramics, some special fabrication methods have been used as we mentioned before.

One of the biggest challenges for the fabrication of the polycrystal $\mathrm{Ca}_{3} \mathrm{Co}_{4} \mathrm{O}_{9}$ ceramics is to control the crystal alignment or the crystal texture. In previous work, Moon J et al. ${ }^{[14]}$ have done some research on the anisotropic thermoelectric properties of substituted $\mathrm{Ca}_{3} \mathrm{Co}_{4} \mathrm{O}_{9}$ bulk system along in-plane direction and out-of-plane direction. The electrical conductivity along in-plane was about four times larger than that along out-of-plane direction, while Seebeck coefficients were similar. Although the thermal conductivity was the highest along the in-plane, the figureof-merit along in-plane was significantly enhanced solely due to the enhanced electrical conductivity. In fact, whether the figure-of-merit along the in-plane in certain system can be enhanced prominently really depends on the orientation level of crystal grains and also on the competition of the enhancement for both electrical and thermal conductivity. In most research work, due to the limitation of measurement for thermal conductivity, sometimes the sample can only be measured along out-of-plane direction, which is usually the pressing direction for sample pellet processing. From common sense, the value of thermal conductivity along this direction will be much higher that the value in in-plane because of the orientation of crystal grains.

\subsection{Improve the thermoelectric properties}

\subsubsection{Optimize the TE performance by metal ion doping}

For practical use, the TE performance of $\mathrm{Ca}_{3} \mathrm{Co}_{4} \mathrm{O}_{9}$ bulk material must be further improved. So far, one approach to improve the TE properties is doping Ca or Co sites with metals such as rare earth alkali metals or alkaline earth metals or heavy metals. The thermoelectric properties of $\mathrm{Ca}_{3} \mathrm{Co}_{4} \mathrm{O}_{9}$ were summarized in Fig 2.3 by $\mathrm{J}$ W Fergus. ${ }^{[15]}$ No matter doped or pure $\mathrm{Ca}_{3} \mathrm{Co}_{4} \mathrm{O}_{9}$, these materials are showing an electrical conductivity of around $10^{4} \mathrm{~S} \mathrm{~m}^{-1}$, a Seebeck coefficient of around $150 \mu \mathrm{V} \mathrm{K} \mathrm{K}^{-1}$ and a thermal conductivity of around $2 \mathrm{~W} \mathrm{~K}^{-1} \mathrm{~m}^{-1}$. In Fig 2.3d, Fergus used a line representing the range of most of the values of figure of merit. 

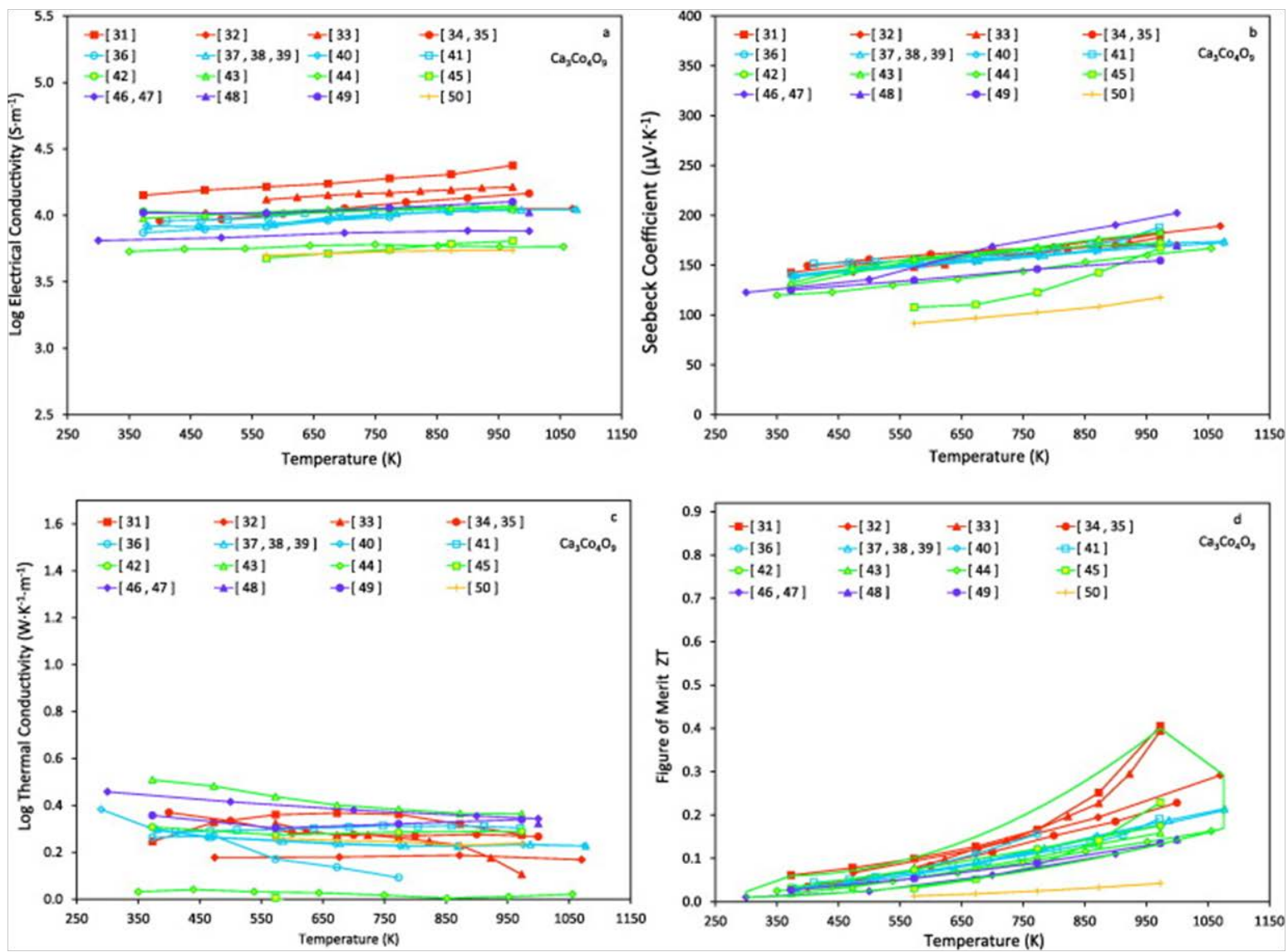

Figure 2. 3 Electrical conductivity (a), Seebeck coefficient (b), thermal conductivity (c) and figure of merit (ZT) (d) for Co349. (The figure is cited from a review, the number 31-50 in the figure refers the reference number in this review. $)^{[15]}$

One dopant commonly used with $\mathrm{Ca}_{3} \mathrm{Co}_{4} \mathrm{O}_{9}$ is bismuth, ${ }^{[15]}$ which has been shown to increase both the electrical conductivity and Seebeck coefficient, as well as decrease the thermal conductivity. The increase in electrical conductivity is usually considered as the attribution to an increase in carrier mobility, rather than carrier concentration. Although bismuth additions have been shown to improve all three properties contributing to the figure-of-merit, the reported figure-of-merits are in middle to upper range of those reported for undoped $\mathrm{Ca}_{3} \mathrm{Co}_{4} \mathrm{O}_{9}$.

The addition that appears to have the greatest impact on the figure-of-merit is silver. The silver as dopant or silver as co-dopant with barium or lutetium in $\mathrm{Ca}_{3} \mathrm{Co}_{4} \mathrm{O}_{9}$ were observed to increase in electrical conductivity, but, in contrast decrease Seebeck coefficient. When combined with a dopant, however, the silver second phase can lead to an increase in both electrical conductivity and thermopower. 

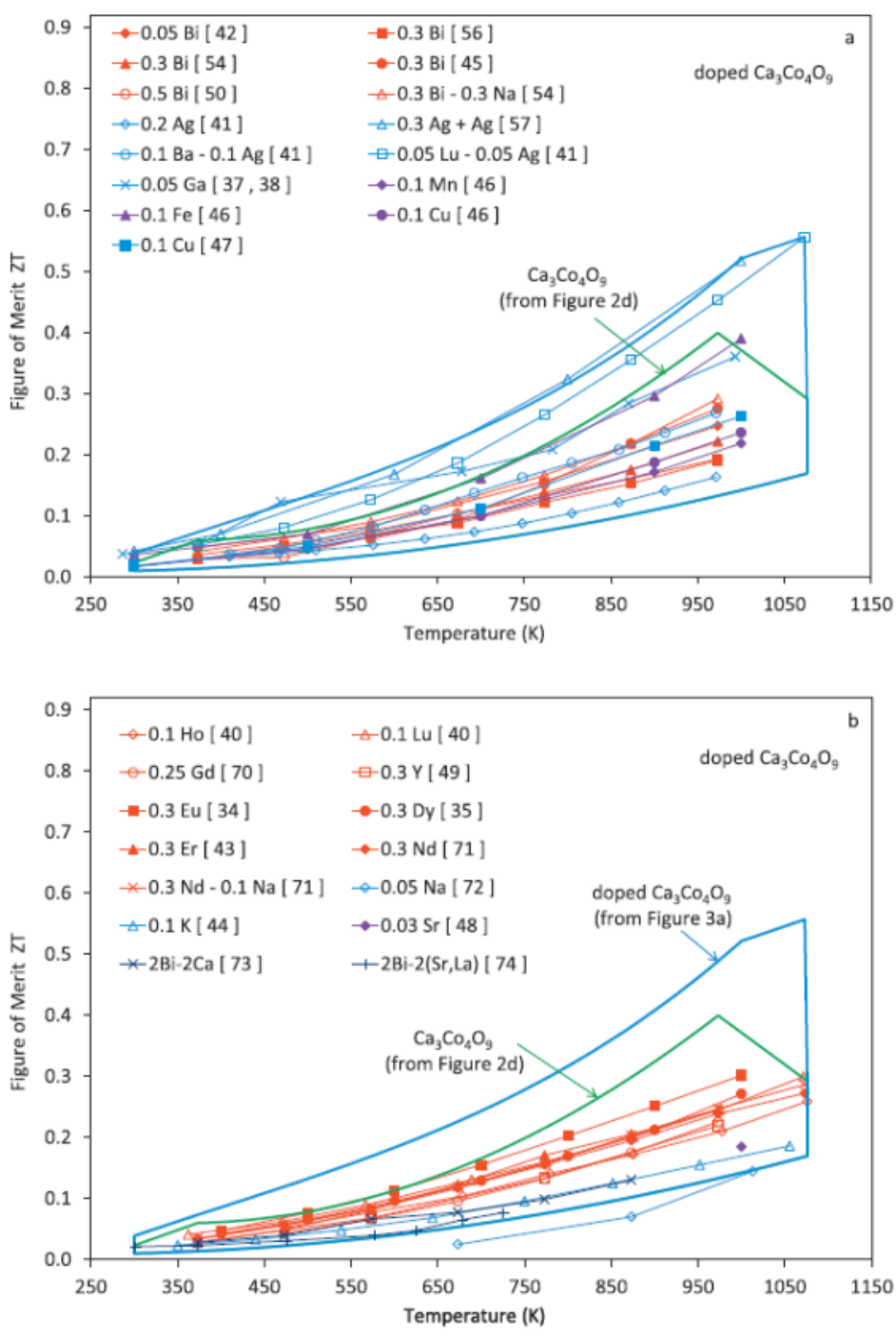

Figure 2. 4 Figure of merit (ZT) for doped $\mathrm{Ca}_{3} \mathrm{Co}_{4} \mathrm{O}_{9} \cdot{ }^{[15]}$

The third dopant, copper, shown in Fig $2.4 \mathrm{a}^{[15]}$ was reported to increase the electrical conductivity, but also decrease the Seebeck coefficient. Heavier transition metal dopants for $\mathrm{Ca}_{3} \mathrm{Co}_{4} \mathrm{O}_{9}$, some of which increases electrical conductivity, but decreases thermopower, others increase both the electrical conductivity and the thermopower. The figure of merit $\mathrm{Ca}_{3} \mathrm{Co}_{4} \mathrm{O}_{9}$ doped with other dopants was shown in Fig 2.4b. The results reported for lanthanum doping are mixed in contradictive conclusion, however, reports for other lanthanide elements are consistent, which lead to an increase in thermopower, but a decrease in electrical conductivity.

\subsubsection{Optimize the TE performance by special fabrication methods}


To optimize the TE performance of $\mathrm{Ca}_{3} \mathrm{Co}_{4} \mathrm{O}_{9}$ ceramics, some special fabrication methods for bulk materials were chosen ${ }^{[3-7,16]}$, such as Hot-Pressing (HP), Cold high pressure pressing, Magnetic Alignment (MA) and Spark Plasma Sintering (SPS) process. All these methods can produce highly grain-aligned and densified Co-based oxide ceramics, as shown in Fig 2.5. These samples were measured to be with increased electrical conductivity and thermopower along inplane. However, there were no data for thermal conductivity along the same direction as electrical conductivity. In another words, the real TE performance is still not known, although the thermopower usually was improved by these special fabrication methods.

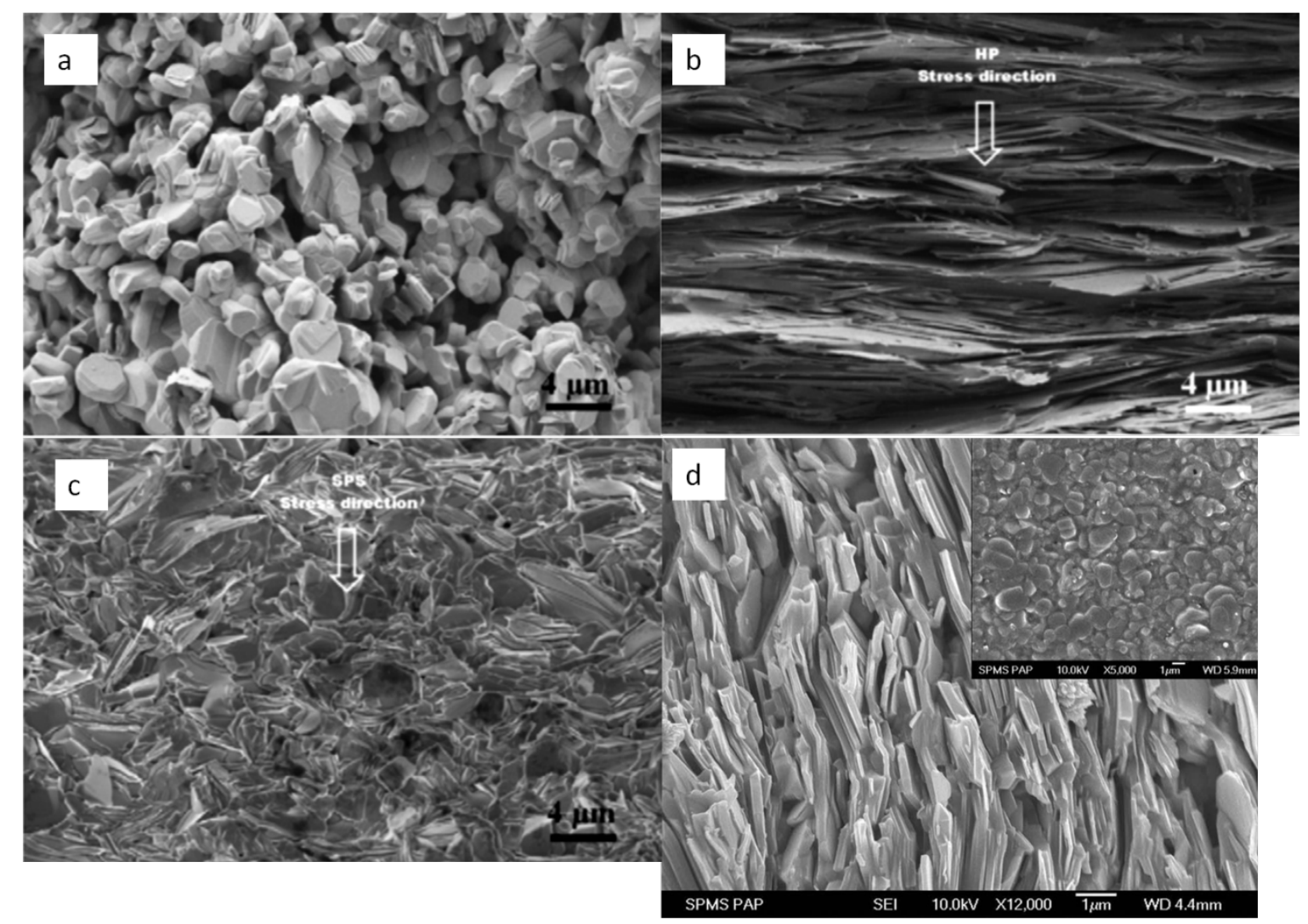

Figure 2. 5 SEM micrographs of fractured surfaces of the $\mathrm{Ca}_{3} \mathrm{Co}_{4} \mathrm{O}_{9}$ samples processed by: ${ }^{[4,16]}$ (a) conventional sintering (CS), (b) hot-pressing (HP), (c) spark plasma sintering (SPS) processing and (d) cold high-pressure fabrication.

\subsubsection{Optimize the TE performance by Minimizing the Thermal Conductivity}

As we know from the equation of figure of merit, to improve the TE performance need to increase Seebeck coefficient and electrical conductivity, but decrease the thermal conductivity. 
However, for certain pure TE material, the value of Seebeck coefficient is almost fixed. To improve the figure-of-merit, the efficient way is to minimize the thermal conductivity contributed by phonon scattering. In many areas of research related to TE materials, this is being attempted by scattering phonons in different frequency ranges using a variety of methods such as mass fluctuation scattering (a mixed crystal, in ternary and quaternary compounds), "rattling” scattering, grain-boundary scattering (due to the size of the grains), and interface scattering in thin films or multilayer systems.

The lattice thermal conductivity $\kappa_{\mathrm{ph}}$ is given by $\kappa_{\mathrm{ph}} \approx(1 / 3)\left(v_{s} C L_{\mathrm{ph}}\right)$, where $v_{s}$ is the velocity of sound, C is the heat capacity, and $L_{\mathrm{ph}}$ is the mean free path of the phonons. ${ }^{[11]}$ At high temperatures, the sound velocity and the heat capacity cannot be controlled by any other factor because their essential temperature-independent character in typical materials. Therefore, the magnitude and the temperature-dependence of $\kappa_{\text {ph }}$ are determined only by the mean free path of the phonons. Therefore, the minimum thermal conductivity will be defined when the mean free path is limited by the inter-atomic distance between the atoms within the crystal.

As we know, until now for $\mathrm{Ca}_{3} \mathrm{Co}_{4} \mathrm{O}_{9}$ materials few researchers have focused on the optimization of the particle size of powder precursor and also grain size of bulk materials. This is worthy to investigate carefully.

\subsubsection{Control the particle size and grain alignment through preparation methods}

There are mainly two types of method for preparation of $\mathrm{Ca}_{3} \mathrm{Co}_{4} \mathrm{O}_{9}$ powder precursor: one commonly used is solid-state reaction and the other is sol-gel method. The solid state reaction can produce powder with big amount, which is convenient for fabrication of material for large device, however, the impurity phase is often observed in the bulk materials. The sol-gel method can be used to obtain uniform chemistry and fine powers by controlling the components with stoichmetric ratio. Sol-gel method is considered as an efficient way to control the grain size in the final sintered ceramics. In recent years, the researchers optimized the sol-gel method and created another method called polymerized method. ${ }^{[3]}$ This method uses citric acid as one component with stoichmetric ratio of metal ions and uses ethylene glycol as solvent and also as a reactant for polymerization during the heating process. The polymers obtained during this process act as an efficient frame to avoid the collapse and hard agglomerates in the system. This 
method was considered to further improve the stability of the sol-gel citrate system for ceramic powder fabrication.

The traditional sol-gel used for $\mathrm{Ca}_{3} \mathrm{Co}_{4} \mathrm{O}_{9}$ preparation is Pechini method. The shape and particle size of as-calcined ceramic powders made by a Pechini-type process are mainly determined by the morphologies of their charred resin intermediates. Tai et al. ${ }^{[1]}$ considered a soft ceramic precursor with high porosity was preferred for making nonagglomerated, fine, mixed-cation oxide powders. In addition to the compositional dependence of polymeric gel, the amounts of organic constituents and additional water content in the precursory mixture also affect the formation of resin intermediates (and the consequent ceramic powders). After very porous resins are obtained, it does not guarantee fine ceramic powder after calcinations without cautious processing. Proper pulverization techniques are definitely required to break the huge "puff” of semiproduct to small loose pieces before calcinations. And also the careful chosen condition for calcinations will also affect the crystal grains and even the thermoelectric properties in the final sintered ceramics.

\subsection{Anisotropic thermoelectric properties}

An unusual anisotropy of thermopower has been observed in $\mathrm{Ca}_{3} \mathrm{Co}_{4} \mathrm{O}_{9}$ single crystal by Tang et al. $^{[18]}$ They found the in-plane thermopower is about twice as big as the out-of-plane thermopower and explained the anisotropy of thermopower was led by the strong anisotropy in the topology of Fermi surface. According to this phenomenon, it is easily understood that the potential anisotropy existed in highly-oriented polycrystalline $\mathrm{Ca}_{3} \mathrm{Co}_{4} \mathrm{O}_{9}$. In 2001, Moon et al. ${ }^{\text {[14] }}$ found in highly textured $\mathrm{Ca}_{3} \mathrm{Co}_{4} \mathrm{O}_{9}$ ceramics both the thermal and electrical conductivity show the difference along in-plane and along out-of-plane. This result is consistent with WiedemannFranz Law about that electric conductivity is usually accompanied by high thermal conductivity.

\subsection{Phase Equilibria in the Ca-Co-O system}

The compound phases of calcium cobalt oxides were dependent on sintering temperature. Woermann et al. ${ }^{[19]}$ have reported the phase equilibria in the system Ca-Co-O system in air, shown in Fig 2.6. The only crystalline phases existing in equilibrium with liquids in the system 


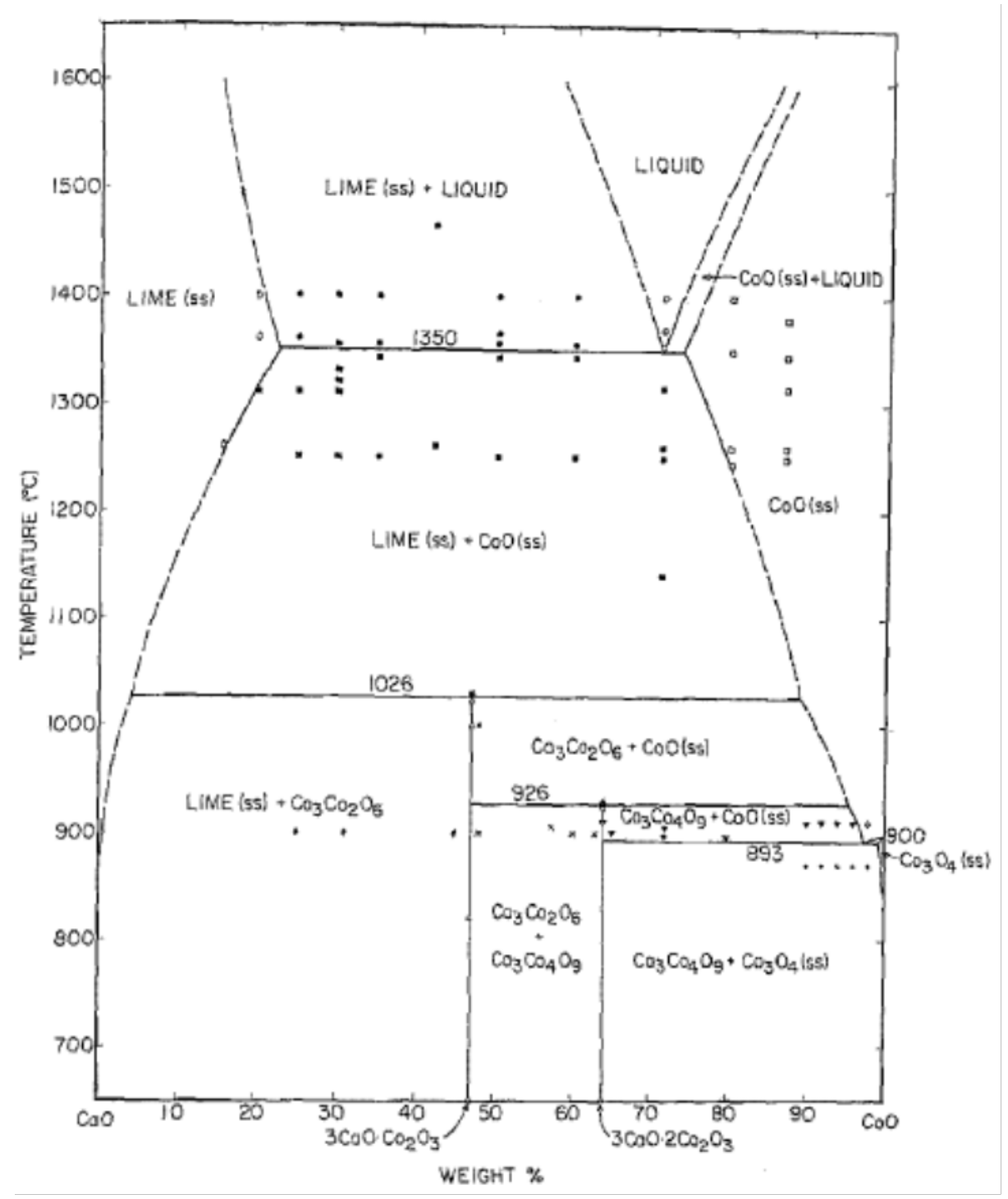

Figure 2. 6 Phase equilibrium diagram for the system Ca-Co-O system in air. ${ }^{[19]}$

are lime solid solution and $\mathrm{CoO}$ solid solution; the eutectic temperature in air is $1623 \mathrm{~K}$. From Fig 2.6, it is clearly seen that at temperatures well below the solidus, two important compounds are present. One has the composition $\mathrm{Ca}_{3} \mathrm{Co}_{4} \mathrm{O}_{9}$, the other is $\mathrm{Ca}_{3} \mathrm{Co}_{2} \mathrm{O}_{6}$. The former composite is stable up to $1179 \mathrm{~K}$ and decomposes above $1179 \mathrm{~K}$ into the $\mathrm{CoO}$ solid solution and a phase of composition $\mathrm{Ca}_{3} \mathrm{Co}_{2} \mathrm{O}_{6}$. The $\mathrm{Ca}_{3} \mathrm{Co}_{2} \mathrm{O}_{6}$ phase is stable up to $1300 \mathrm{~K}$ in air, where it decomposes to lime solid solution and $\mathrm{CoO}$ solid solution. 
Tashashi et al. ${ }^{[20]}$ observed the sintering temperature dependence of thermoelectric performance and crystal phase of calcium cobalt oxides. They synthesized polycrystalline samples with a molar ratio of $\mathrm{Ca}: \mathrm{Co}=3: 4$ and sintered them at 1073-1373K. For the sample with $\mathrm{Ca}_{3} \mathrm{Co}_{2} \mathrm{O}_{6}$ phase, which was sintered at $1273 \mathrm{~K}$, the Seebeck coefficient was greater than $600 \mu \mathrm{V} / \mathrm{K}$ at $373 \mathrm{~K}$, and decreased with the increasing measurement temperature, but remained above $200 \mu \mathrm{V} / \mathrm{K}$ at $873 \mathrm{~K}$. For the sample sintered at $1327 \mathrm{~K}$, in which the decomposition phase of $\mathrm{Ca}_{3} \mathrm{Co}_{2} \mathrm{O}_{6}$ formed, the Seebeck coefficient was greater than $200 \mu \mathrm{V} / \mathrm{K}$ and increased gradually with the increasing measurement temperature up to $600 \mathrm{~K}$ to reach a maximum value of $274 \mu \mathrm{V} / \mathrm{K}$, and then began to decrease. Although $\mathrm{Ca}_{3} \mathrm{Co}_{2} \mathrm{O}_{6}$ had a Seebeck coefficient greater than that of $\mathrm{Ca}_{3} \mathrm{Co}_{4} \mathrm{O}_{9}$, but a large power factor was not obtained because the resistivity was an order of magnitude higher than that of $\mathrm{Ca}_{3} \mathrm{Co}_{4} \mathrm{O}_{9} .2 .7$ Other calcium cobalt oxides

In calcium cobalt oxide family, except Co349 there are another two types of composites: one is $\mathrm{Ca}_{2} \mathrm{Co}_{2} \mathrm{O}_{5}$, the other is $\mathrm{Ca}_{3} \mathrm{Co}_{2} \mathrm{O}_{6}$.

\subsubsection{The structure and preparation condition of $\mathrm{Ca}_{2} \mathrm{Co}_{2} \mathrm{O}_{5}$}

$\mathrm{Ca}_{2} \mathrm{Co}_{2} \mathrm{O}_{5}$ used to be described by Vidyasagar et al. ${ }^{[21]}$ as alternated stacking of octahedral $\left[\mathrm{CoO}_{2}\right]_{\infty}$ and square-pyramidal $[\mathrm{CoO}]_{\infty}$ layers separated by $[\mathrm{CaO}]_{\infty}$ layers. The $\mathrm{X}$-ray diffraction pattern of $\mathrm{Ca}_{2} \mathrm{Co}_{2} \mathrm{O}_{5}$ has been indexed on the basis of an orthorhombic unit cell with a $=11.12(1)$, $\mathrm{b}=10.74(1)$, and $\mathrm{c}=7.48(1) \AA$. However the structure of $\mathrm{Ca}_{2} \mathrm{Co}_{2} \mathrm{O}_{5}$ in the form of textured ceramics prepared by co-precipitation and spark plasma sintering ${ }^{[22-23]}$ has been reported with a layered structure (shown in Fig 2.7a) similar to that of Co349. ${ }^{[24]}$ Recently Boullay et al. ${ }^{[25]}$ borrowed precession electron diffraction to determine the structure of $\mathrm{Ca}_{2} \mathrm{Co}_{2} \mathrm{O}_{5}$ thin film and found the structure of $\mathrm{Ca}_{2} \mathrm{Co}_{2} \mathrm{O}_{5}$ was totally different from those previously reported. In their work, a well-crystallized epitaxial $\mathrm{Ca}_{2} \mathrm{Co}_{2} \mathrm{O}_{5}$ thin film has been deposited on $\mathrm{NdGaO} 3$ substrate by the pulsed laser deposition technique. Its average brownmillerite structure (its XRD result is shown in Fig 2.7b) has been observed with parameters at $a \approx a_{p} \sqrt{2}, b \approx 4 a_{p}$, and $c \approx a_{p} \sqrt{2}$.

The contradiction in the structure determination of $\mathrm{Ca}_{2} \mathrm{Co}_{2} \mathrm{O}_{5}$ is not very clear. However, according to the previous references about $\mathrm{Ca}_{2} \mathrm{Co}_{2} \mathrm{O}_{5}$, the preparation methods for this compound are various for different researchers. In the work reported by Boullay et al. ${ }^{[25]}$, targets with a 

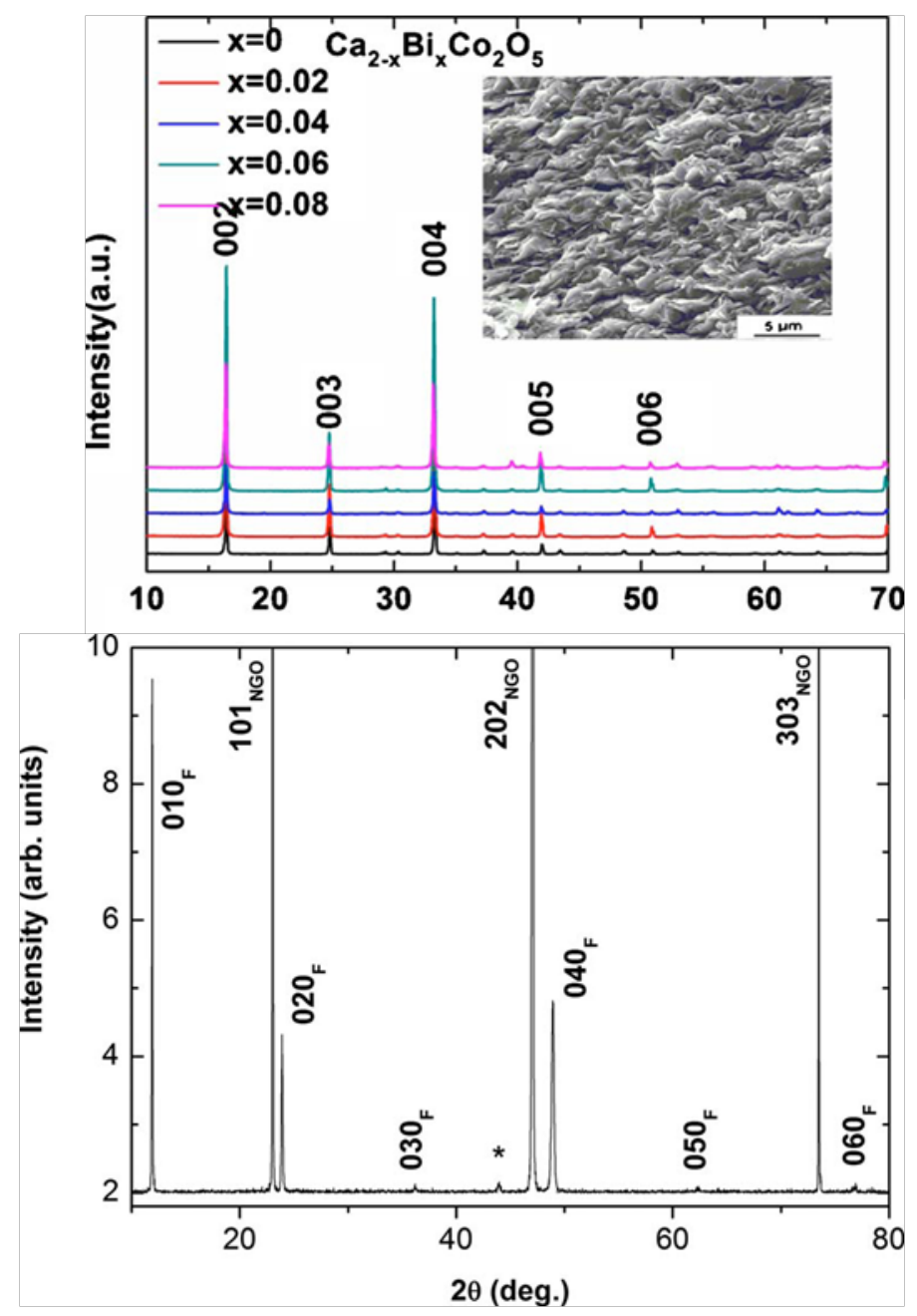

Figure 2. 7 (a) XRD patterns of $\mathrm{Ca}_{2-\mathrm{x}} \mathrm{Bi}_{\mathrm{x}} \mathrm{Co}_{2} \mathrm{O}_{5}$ samples. The insert shows SEM picture of $\mathrm{Ca}_{1.98} \mathrm{Bi}_{0.02} \mathrm{Co}_{2} \mathrm{O}_{5}$ sample, ${ }^{[23]}$ (b) Typical XRPD pattern of a calcium cobaltite thin film $(\mathrm{Ca} / \mathrm{Co}=1)$ grown on NdGaO3 under optimized conditions. The peak labeled with a star is generated by the sample holder. ${ }^{[25]}$

$\mathrm{CaCoO}_{x}$ composition were prepared by solid state reaction first. During this process, the powder mixture was calcined at $900^{\circ} \mathrm{C}$ and the pressed pellets were sintered at $1150^{\circ} \mathrm{C}$. Then thin films of $\mathrm{Ca}_{2} \mathrm{Co}_{2} \mathrm{O}_{5}$ were grown by PLD method. In the research by Lan et al. ${ }^{[23]}$ the precipitation method was used to prepare the precursor and the precursor was calcined at $800^{\circ} \mathrm{C}$ for $2 \mathrm{~h}$ in air, and the bulk sample was sintered in a SPS apparatus at $950^{\circ} \mathrm{C}$ for 5 min with a pressure of $40 \mathrm{MPa}$. Right now in XRD database the $\mathrm{x}$-ray diffraction of powder $\mathrm{Ca}_{2} \mathrm{Co}_{2} \mathrm{O}_{5}{ }^{[26]}$ was indexed according to the research results by Vidyasagar et al. ${ }^{[21]}$. Vidyasagar et al. synthesized $\mathrm{Ca}_{2} \mathrm{Co}_{2} \mathrm{O}_{5}$ powder by decomposing the precursor in an oxygen atmosphere at $650^{\circ} \mathrm{C}$ for $6 \mathrm{~h}$. The chemical analyses showed that cobalt was in the 
3+ state and calcium and cobalt were in the required ratio.2.7.2 The structure and preparation condition of $\mathrm{Ca}_{3} \mathrm{Co}_{2} \mathrm{O}_{6}$

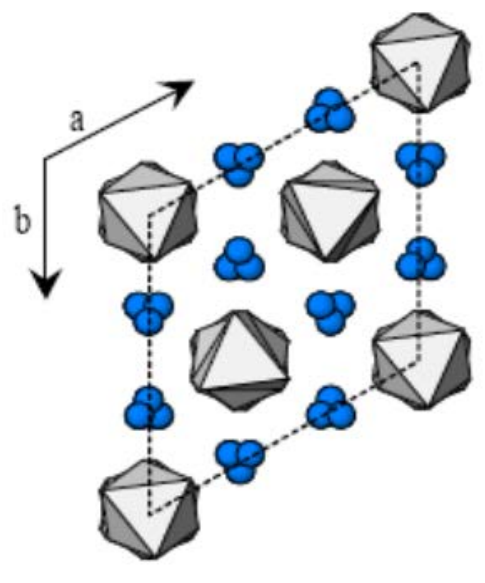

a

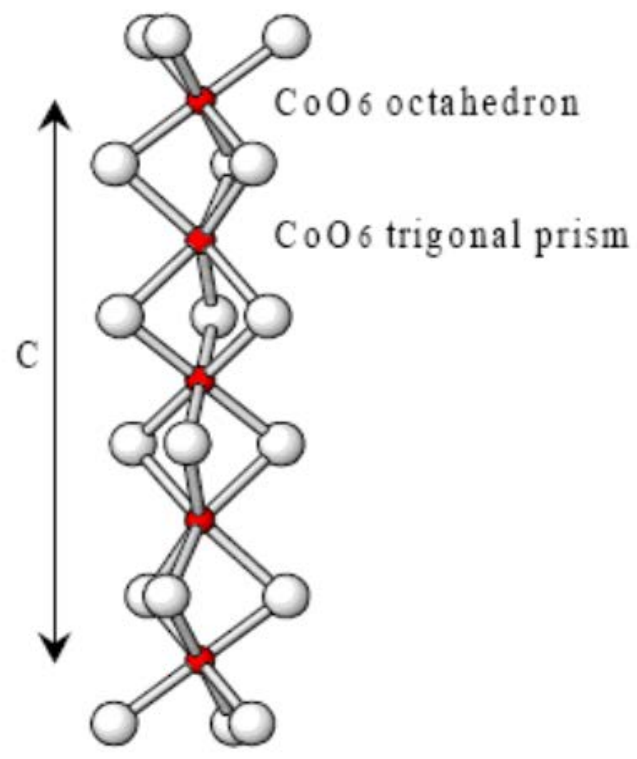

$\mathrm{b}$

Figure 2. 8 (a) Projection onto (001) of the $\mathrm{Ca}_{3} \mathrm{Co}_{2} \mathrm{O}_{6}$ structure showing the triangular arrangement of the chains, (b) $\left[\mathrm{Co}_{2} \mathrm{O}_{6}\right]_{\infty}$ chain evidencing the alternation of cobalt in octahedral and prismatic coordination.

In the Ca-Co-O system, the compound $\mathrm{Ca}_{3} \mathrm{Co}_{2} \mathrm{O}_{6}$ has been known for a long time. ${ }^{[19]}$ However, only its diffraction pattern had been described in the literature until in 1996 by Fjellvag et al. ${ }^{\text {[26] }}$ its crystal structure by ab initio methods from powder X-ray diffraction data was solved and refined from powder neutron diffraction data. $\mathrm{Ca}_{3} \mathrm{Co}_{2} \mathrm{O}_{6}$ crystallizes in space group $R-3 c, Z=6$ (hexagonal setting) and $a=907.93(7)$ and $c=1038.1(1) \mathrm{pm}$. The Co atoms form alternating, face-sharing coordination polyhedral of trigonal prisms and oxtahedra. In this way onedimensional Co-O chains are formed that run along the trigonal axis. The chains are separated by eight coordinated calcium atoms. The separation between Co atoms within the chains are 259.5 pm which suggest metal-metal interactions. Based on interatomic Co-O distances and bond strength considerations, it was proposed that either charge or spin ordering of the different Co species occurred within the chains. ${ }^{[26]}$ Figure 2.8 shows that the rhombohedral structure of this phase consists of $\left[\mathrm{Co}_{2} \mathrm{O}_{6}\right]_{\infty}$ chains running along the $c$ axis of the corresponding hexagonal cell 
(Fig. 2.8a) and one $\mathrm{CoO}_{6}$ octahedron chain alternating with one $\mathrm{CoO}_{6}$ trigonal prism along $c$ (Fig $2.8 b) .{ }^{[27]}$

Field-induced magnetic transitions and magnetic properties of $\mathrm{Ca}_{3} \mathrm{Co}_{2} \mathrm{O}_{6}$ with ferromagnetic Ising chains had been studied by Kageyama et al. ${ }^{[28-29]}$ From the extremely anisotropic behavior in its magnetization at low temperatures and in the temperature dependence of magnetic susceptibility, it was suggested that an octahedral was nonmagnetic, whereas a trigonal prismatic had a fictitious spin with large single-ion anisotropy. And at low temperatures below 5K, multisteps were observed in the magnetization, suggesting the existence of various magnetic structures.

Maignan et al. ${ }^{[27]}$ has done some research on $\mathrm{Ca}_{3} \mathrm{Co}_{2} \mathrm{O}_{6}$ single crystals and confirmed the previous observations ${ }^{[28-29]}$ on polycrystalline samples. It consists of a triangular lattice of ferromagnetic $\left[\mathrm{Co}_{2} \mathrm{O}_{6}\right]$ chains antiferromagnetically coupled.

Kageyama et al. ${ }^{[28-29]}$ used a solid state reaction method to obtain the polycrystalline of $\mathrm{Ca}_{3} \mathrm{Co}_{2} \mathrm{O}_{6}$. The powder mixtures of $\mathrm{CaCO}_{3}$ and $\mathrm{CoO}$ were ground and calcined in air at $1173 \mathrm{~K}$ for one day. Then, the sample was reheated at $1273 \mathrm{~K}$ in air for a week with intermediate grindings. The powder sample was separated into two parts, one of which was soaked into the epoxy and then magnetic field of $H=8 \mathrm{~T}$ was quickly applied to align the sample along the caxis. The other was kept in the absence of magnetic field after soaked into the epoxy to form a nonoriented sample.

The $\mathrm{Ca}_{3} \mathrm{Co}_{2} \mathrm{O}_{6}$ polycrystalline sample was made by Fjellvag et al. ${ }^{[26]}$ through a wet chemical method, i.e. $\mathrm{CaCO}_{3}, \mathrm{Co}\left(\mathrm{CH}_{3} \mathrm{COO}\right)_{2} \cdot 4 \mathrm{H} 2 \mathrm{O}$ and citric acid monohydrate was mixed and dissolved in distilled water. The mixture solution was dehydraeted at $450 \mathrm{~K}$. Most of the carbonaceous species were thereafter removed by incineration at $720 \mathrm{~K}$ for a few hours. The final sample pellets were heated at $1273 \mathrm{~K}$ for 1 week with one intermittent grinding.

Both in solid state method or wet chemical method, the final annealing temperature for sample was $1273 \mathrm{~K}$. 


\section{Chapter 3: Effect of Calcinations Temperature for Powder Precursor on the Thermoelectric Properties of $\mathrm{Ca}_{3} \mathrm{Co}_{4} \mathrm{O}_{9}$ Ceramics}

\subsection{Objective}

Calcine the $\mathrm{Ca}_{3} \mathrm{Co}_{4} \mathrm{O}_{9}$ (Co349) powder precursor at different temperature, observe the microstructure by SEM and TEM, measure the purity of crystal phase in final sintered bulk sample by XRD. Since the improvement of electrical conductivity is usually accompanied by the increasing of thermal conductivity, the optimized calcinations condition is needed to find a best figure of merit.

This study was proposed to determine the optimal calcinations temperature for Co349 powder precursors. A series of experiments were conducted to examine the effects of calcinations temperature of powder precursor upon the electrical resistivity and the thermal conductivity, and also upon microstructure of bulk Co349 material. Combining the microstructure and the thermoelectric property of final sintered body, the calcinations condition was optimized. Since the microstructure of powder precursor has effect on the microstructure of final bulk material, the powder precursor preparation was made also by an optimized sol-gel method according to the previous reference.

\subsection{Introduction}

Compared with those conventional thermoelectric materials such as metal chalcogenides, transition metal disilicates, and silicon-germanium alloys, layered metal oxides have attracted much attention since the discovery of high TE performance in $\gamma-\mathrm{Na}_{x} \mathrm{CoO}_{4}$ by Terasaki et al.. ${ }^{[1]}$ However, due to the volatility and poor resistance to humidity practical application of $\mathrm{NaCo}_{2} \mathrm{O}_{4}$ for power generation is quite limited. Another cobaltite Co349 was considered as most promising candidate for a p-type high temperature TE material due to its stability and low toxicity at high temperature. The crystal structure of Co349 is a misfit-layered structure consisting of an electrically conductive $\mathrm{CdI}_{2}$-type $\mathrm{CoO}_{2}$ layer and an insulating $\mathrm{Ca}_{2} \mathrm{CoO}_{3}$ rocksalt-type layer, which result in $Z T=0.83$ at $973 \mathrm{~K}$ for the single crystal. ${ }^{[2]}$ However the $\mathrm{ZT}$ of the polycrystalline bulk Co349 is still at a relatively low level. As we noticed, high-efficiency thermoelectric conversion requires simultaneously low electrical resistivity and low thermal conductivity. To optimize the TE performance of Co349 ceramics, some special fabrication methods for bulk materials were chosen ${ }^{[3-7]}$, such as Hot-Pressing (HP), Cold high pressure pressing, Magnetic Alignment (MA) and Spark Plasma Sintering (SPS) process. However, according to the Wiedemann-Franz Law high electric conductivity is usually accompanied by high thermal conductivity in highly textured polycrystalline samples. As a result the fabrication of fine microstructure in a sintered body of Co349 is the only remaining choice for reduction of phonon contribution to thermal conductivity and enhancing the thermoelectric conversion efficiency. This could be realized by sol-gel method to prepare ceramic powder precursor. 
By traditional Pechini ${ }^{[30]}$ method, hard crystallite agglomerates are frequently formed during the combustion process. In order to fabricate fine microstructure in a sintered ceramic body, according to the reference, ${ }^{[31]}$ it is very necessary to properly control the processing parameters for powder precursor, which include mass ratio of citric acid (CA) and ethylene glycol (EG), pulverization before calcinations, and the calcinations conditions. In our work, we use 4:5 molar ratio of CA and EG, which fall within theoretical gelling region ${ }^{[17]}$. A highly porous polymeric resin was formed by charring the metal nitrate-CA/EG gel mixture. This organic foam is an ideal skeleton to prevent forming hard agglomerates. We found the calcinations condition such as temperature was very critical to get a final sintered body with fine microstructure.

Tahashi et. al. ${ }^{[20]}$ have reported the effects of sintered temperature on high temperature thermal properties of Co349. Unfortunately, published literature gives no answer to the question of how the calcination temperature for powder precursors influences the microstructure and thermoelectric properties in the final sintered body. This study was proposed to determine the optimal calcination temperature for Co349 powder precursors. A series of experiments were conducted to examine the effects of calcinations temperature of powder precursor upon the electrical resistivity and the thermal conductivity, and also upon microstructure of bulk Co349 material.

\subsection{Experimental procedures}

The Co349 powder precursor was prepared by dissolving citric acid and stoichiometric amounts of calcium nitrate tetrahydrate and cobalt nitrate hexahydrate in de-ionized water in a beaker, followed by addition of ethylene glycol. The ratio of citric acid and ethylene glycol was optimized with molar ratio of 4:5 to ensure a soft and porous resin intermediate obtained. ${ }^{[17,31]}$ The above mixture was first heated to $\sim 50^{\circ} \mathrm{C}$ and stirred on a hot stage until complete dissolution, and then heated to $\sim 90^{\circ} \mathrm{C}$ to remove most of water from the solution. Then the sample was placed in an oven, where the sample was charred and combusted into powder at $500^{\circ} \mathrm{C}$ for $2 \mathrm{~h}$. The obtained powder was completely ground and calcined at different temperatures such as $650^{\circ} \mathrm{C}, 700^{\circ} \mathrm{C}, 750^{\circ} \mathrm{C}$, or $800^{\circ} \mathrm{C}$ for $4 \mathrm{~h}$ with oxygen flow. The calcined powder was ground and mixed with $1 \%$ PVDF as binder. The appropriate amount of powder mixture was loaded into a square shape dry pressing die with 1 inch of length of its size and was immediately pressed at $35000 \mathrm{LB}$ (approximately $930 \mathrm{MPa}$ ) to obtain a circle shape pellet with thickness at $\sim 2 \mathrm{~mm}$. The obtained pellets were first annealed at $600^{\circ} \mathrm{C}$ for $3 \mathrm{~h}$ to burn out the binder and then sintered at $920^{\circ} \mathrm{C}$ for $12 \mathrm{~h}$ in tube furnace with oxygen flow.

The final sintered bodies with calcinations temperature of $650^{\circ} \mathrm{C}, 700^{\circ} \mathrm{C}, 750^{\circ} \mathrm{C}$, and $800^{\circ} \mathrm{C}$ were represented by Co349-650, Co349-700, Co349-750, and Co349-800, respectively. The phase identification of the obtained samples was made by X-ray diffraction (Bruker D8 Discovery) with $\mathrm{Cu} \mathrm{K}_{\alpha}$ radiation at room temperature. Both the particle morphology for powder and the surface micrograph for bulk samples were investigated by a field emission scanning electron microscope (FE-SEM; JEOL JSM-7600F). 
Samples were cut into $2 \mathrm{~mm} \times 2 \mathrm{~mm} \times 8 \mathrm{~mm}$ bars from the pellet discs for the Seebeck coefficient $(S)$ and electrical resistivity $(\rho)$ measurements. Both $S$ and $\rho$ for all the pellets were simultaneously measured by the standard four-probe dc method (LINSEIS, LSR-3 Seebeck) in helium atmosphere over the temperature range from room temperature to $700^{\circ} \mathrm{C}$. The measurements were carried out along the pressed plane (in-plane). Another pellet disc was used directly for the thermal diffusivity $\alpha(\mathrm{T})$ and specific heat $C_{\mathrm{p}}(\mathrm{T})$ measurement by the laser flash method (LINSEIS, LFA 1000) along the pressing (out-of-plane) direction, i.e., perpendicular to the pressed plane. For $C_{\mathrm{p}}(\mathrm{T})$ measurement we also compare the result with that measured by a differential scanning calorimetry (DSC) method. The results for $C_{\mathrm{p}}(\mathrm{T})$ measured by two different methods are almost same. Then we choose the former method since it can be done simultaneously with $\alpha(\mathrm{T})$ measurement. The thermal conductivity $\kappa$ of the sample was calculated from $\alpha(\mathrm{T}), C_{\mathrm{p}}(\mathrm{T})$, and density $D$ using $\kappa=\alpha C_{\mathrm{p}} D$.

\subsection{Results and discussion}

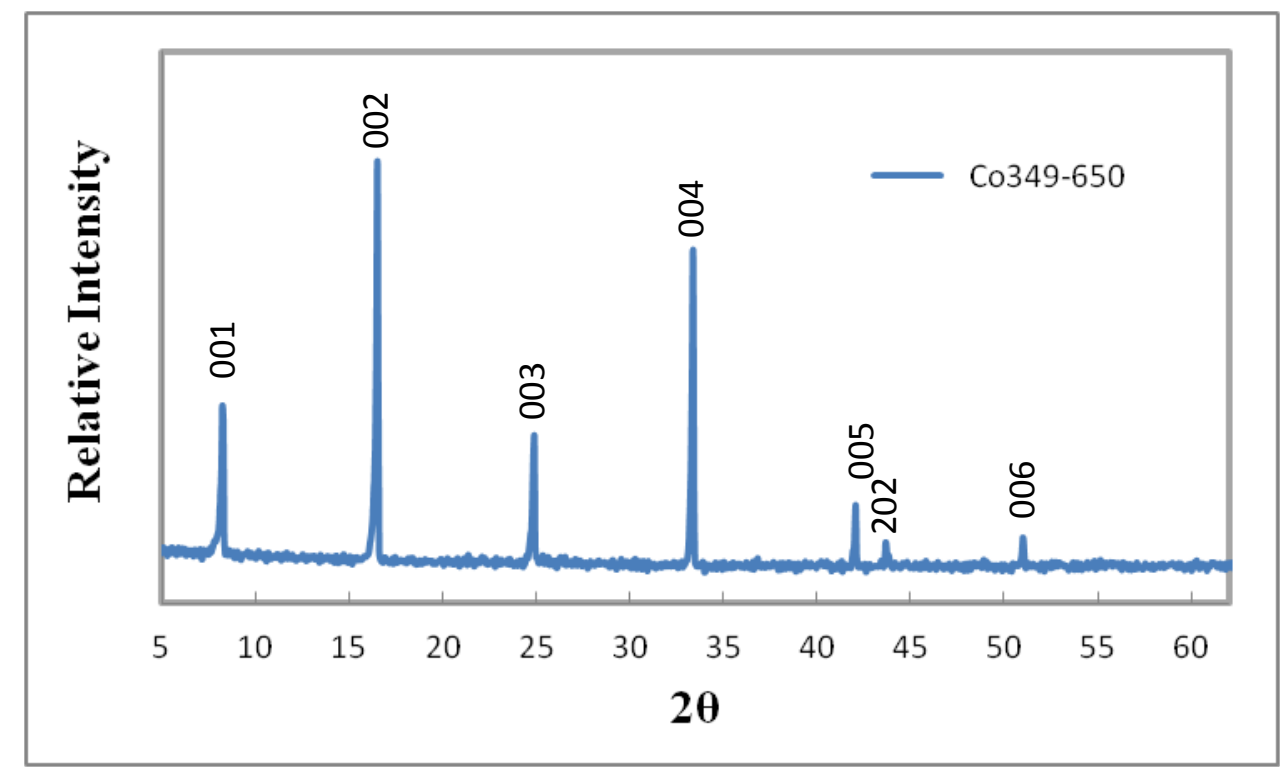

Figure 3. $1 \mathrm{X}$-ray diffraction pattern of Co349 pellet made by powder which was calcined at $650^{\circ} \mathrm{C}($ the pressed pellet was sintered at $920^{\circ} \mathrm{C}$ for $12 \mathrm{~h}$ ).

Oxygen-rich atmospheres were used during calcinations procedure for powder precursor and also in sintering process for bulk materials not only to ensure a clean burnout of organics, but also to enhance the formation of Co349 phase at the lower temperature. This was verified by the result of the X-ray diffraction for the sample Co349-650 and it was found that even at lower calcinations temperature of $650^{\circ} \mathrm{C}$, the final sintered body was composed of Co349 phase without any impurity. Figure 3.1 shows the XRD pattern of Co349 pellet made by powder which 
was calcined at $650^{\circ} \mathrm{C}$ for $4 \mathrm{~h}$ in tube furnace with oxygen atmosphere. All the diffraction peaks can be indexed to the Co349 phase with monoclinic symmetry. ${ }^{[2]}$ The diagram shows a good crystallization of the phase with sharp peaks. The XRD peaks corresponding to $\{00 l\}$ planes are prominent, indicating a good grain alignment in the polycrystalline sample due to the uniaxial pressure. This classical diffraction patterns are very similar to those reported data for oriented Co349 by a combination of magnetic alignment (MA) process and spark plasma sintering (SPS) treatment. ${ }^{[5]}$

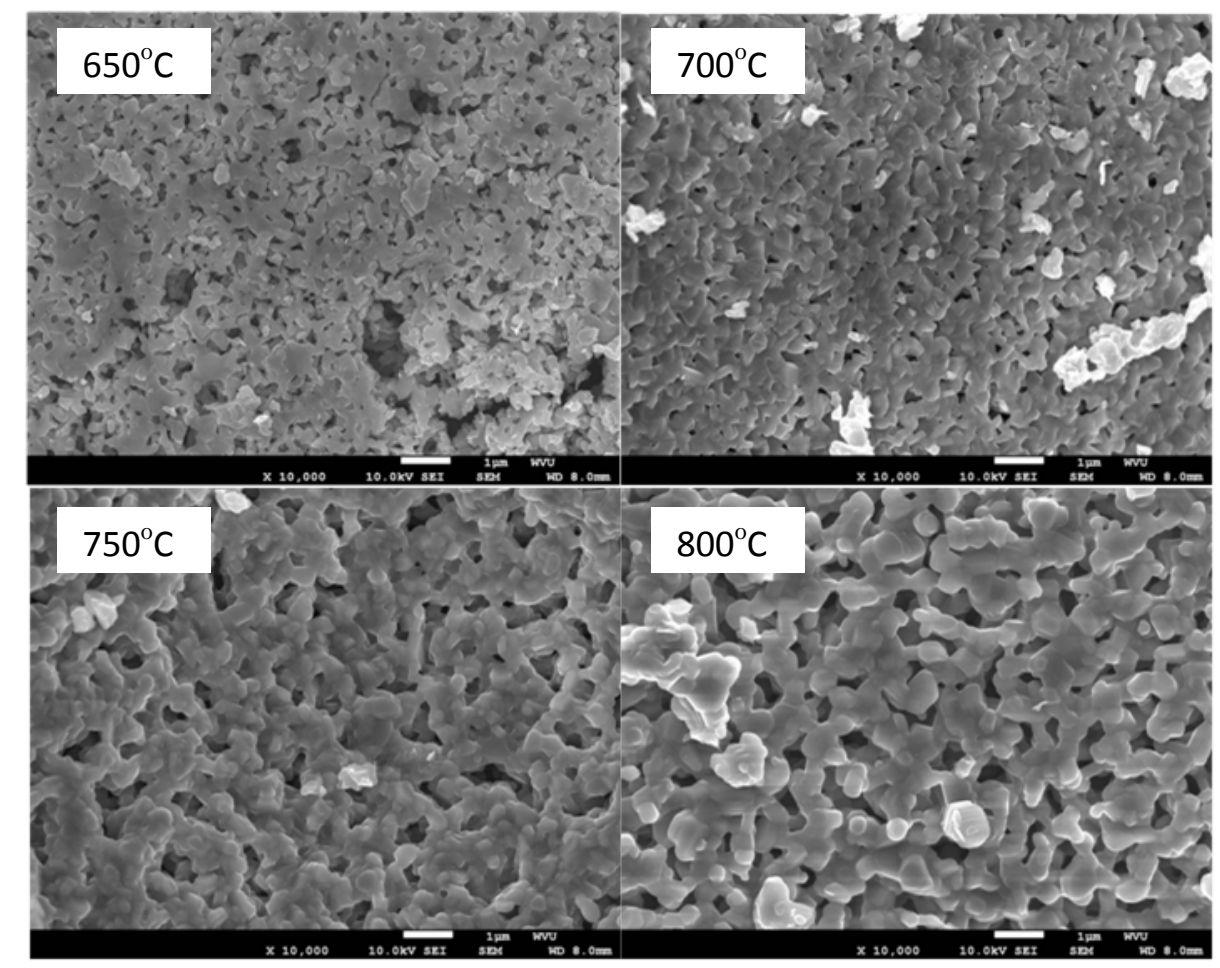

Figure 3. 2 SEM images (scale bar represents $1 \mu \mathrm{m}$ ) for Co349 powder calcined at different temperature: $650^{\circ} \mathrm{C}, 700^{\circ} \mathrm{C}, 750^{\circ} \mathrm{C}$, and $800^{\circ} \mathrm{C}$.

We have examined the effect of calcination temperature upon the particle morphology and particle size distribution both in powder and bulk samples. Figure 3.2 show the SEM measurements for Co349 powder precursor calcined at different temperature. In Fig 3.2, group 1 including A1, B1, C1 and D1 show the SEM observations for unground Co349 powder calcined at $650^{\circ} \mathrm{C}, 700{ }^{\circ} \mathrm{C}, 750^{\circ} \mathrm{C}$ and $800^{\circ} \mathrm{C}$, respectively. For non-ground powder, we found that the particle size increased with increasing calcinations temperature and rigid agglomerates started to be formed when the powders were calcined at $700^{\circ} \mathrm{C}$, which is clearly seen in Fig 3.3. With increasing the calcinations temperature, the plate-like particle starts to form. Higher the temperature used, thicker the plate obtained. Especially after calcined at $800^{\circ} \mathrm{C}$, the particle has around $300 \mathrm{~nm}$ of the diameter and about 150nm of the thickness. 
After the powders were pulverized (as shown in Fig 3.2), the connections among particles obtained during the calcinations procedure were almost broken, but the granules were formed, which was necessary for the pelletizing process later. Higher the calcinations temperature, larger granules could be formed in the powder although most granules were still small. Also, it is noticed that the completeness of the particle was destroyed, and non-broken particle is hardly seen in the area of SEM image. In the previous works, most researchers have used intermittent grinding to obtain the uniformity of the ceramic system, which will make sure the pure phase in the final sintered bodies; however no one has mentioned about the effect of grinding on the particle size or the particle completeness. In our work, we used an optimized sol-gel method to fabricate the Co349 precursor, which is uniform already even without the grinding process before the pelletizing procedure. This result will be displayed in the following chapter.

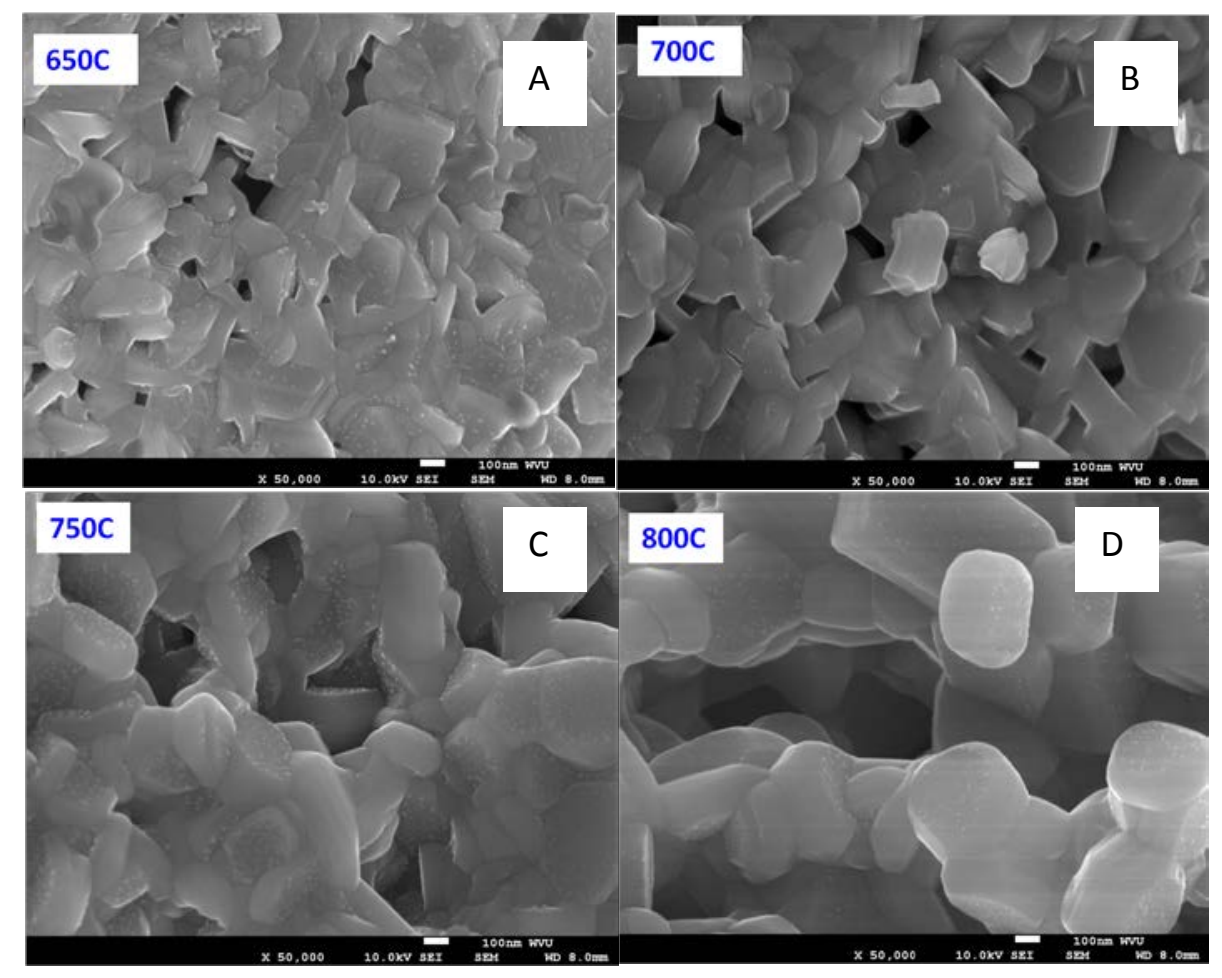

Figure 3. 3 SEM images (scale bar represents $100 \mathrm{~nm}$ ) for Co349 powder calcined at different temperature: (A) $650^{\circ} \mathrm{C}$, (B) $700^{\circ} \mathrm{C}$, (C) $750^{\circ} \mathrm{C}$, and (D) $800^{\circ} \mathrm{C}$.

As mentioned before, the ratio of citric acid and ethylene glycol was optimized according to the previous work ${ }^{[17,31]}$ in order to obtain the most porous resin intermediate with chelated metal cations. When the initial mixture was heated in box furnace, two chemical reactions were involved: (1) chelation between complex cations and citric acid, and (2) polyesterification of excess hydroxycarboxylic acid with glycol. This procedure is similar to the polymerized complexed (PC) method proposed by Katsuyama S. et al. ${ }^{[3]}$ In their PC method citric acid and metal acetate were directly dissolved in ethylene glycol without using de-ionized water. The 
formation of the polymer between ethylene glycol and metal-citrate complexes was happening when the temperature of the solution was gradually increased to $523 \mathrm{~K}$ (i.e. $250^{\circ} \mathrm{C}$ ). For our systems, we chose metal nitrate hydrates instead of metal acetate hydrates and these metal nitrates would be easily decomposed at lower temperature under acidic condition, which would break down the uniformity of the solution. Therefore we moved the mixture from the hot stage into the oven after removing most water in the solution and heated rapidly to $500^{\circ} \mathrm{C}$, where the decomposition of the metal nitrates, the formation of polymer between ethylene glycol and metal-citrate complexes, and the breakdown of the polymer were happened in rapid sequence.
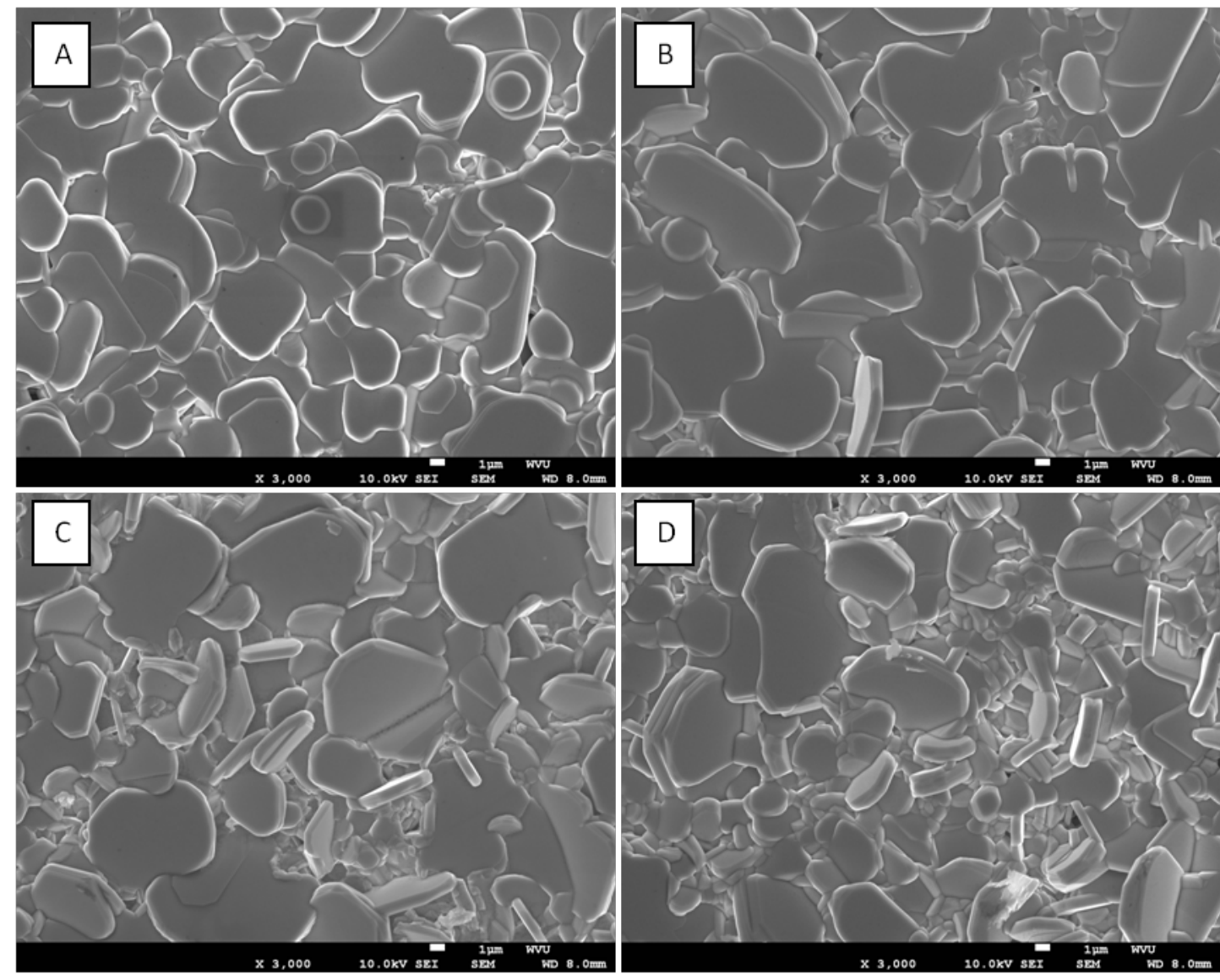

Figure 3. 4 SEM micrographs measured on pressed surface corresponding to those sintered bodies: (A) Co349-650, (B) Co349-700, (C) Co349-750, and (D) Co349-800, respectively. All the pellets were annealed at $920{ }^{\circ} \mathrm{C}$ for 12 hrs.

Figure 3.4 show a comparison among the SEM micrographs taken from the pressed surface of the final sintered bodies. Images A, B, C and D are corresponding to sample Co349-650, Co349700, Co349-750, and Co349-800, respectively. All the samples were sintered at $920^{\circ} \mathrm{C}$ for 12 hrs with oxygen flow. Almost all the sample surfaces were composed of disk-shape grains. The grain 
size distribution on the pressed surface tends to be uniform on the sample Co349-650 and Co349-700 and most grains are with size in the range of 5-10 $\mu \mathrm{m}$, as shown in Fig 3.4A and 3.4B. However, the grain growth on the pressed surface starts to exhibit a bimodal distribution with increasing the calcination temperature to $750^{\circ} \mathrm{C}$ and the surface of the sample appears plenty of small grains surrounded by larger grains. This phenomenon becomes more obvious on the sample Co349-800 (shown in Fig 3.4D), where the size of larger grains is greater than $60 \mu \mathrm{m}$, however the small grains are even less than $1 \mu \mathrm{m}$. Another interesting phenomenon is, almost all the grains on the pressed surface of Co349-650 lay down with disk face perpendicular to the pressing direction. But the other three sample exhibit some disks standing up with disk face parallel to the pressing direction. Furthermore, with increasing the calcinations temperature for powder precursor more grains stand up on the pellet surface.

Figure 3.5 show a comparison of SEM images taken from the fractured cross-sections of those sintered bodies: Co349-650, Co349-700, Co349-750, and Co349-800. The fractured surface is roughly parallel to the pressing direction, indicated by the arrows shown on the images. It is clear that most grains in Fig 3.5 exhibit a disk-like shape, which is consistent with phenomenon observed on the pressed surface; however, these grains are not preferentially aligned along the pressing direction like the samples made by those special methods such as HotPressing, Magnetic Alignment, Spark Plasma Sintering process and etc. ${ }^{[3-7]}$ Furthermore, with increasing the calcinations temperature for powder precursor the grain size and size distribution in the final sintered bodies display a very complicated trend. Firstly, only in sample Co349-650 the grain size are relatively large and size distribution is uniform, which are consistent with the SEM result measured on the pressed surface. Secondly, in samples Co340-750 and Co349-800 there are huge grains with size among 50-100 $\mu \mathrm{m}$ and these grains are almost 10 times larger than the smaller ones inside the sample. Moreover, higher the calcinations temperature, more huge grains generated in the final sintered body. Third, although Co349-700 is free of huge grains, the grain size is much smaller than that in sample Co349-650. At the first glance, this complicated phenomenon is hard to be understood. However, if we compare this result with that shown in Fig 3.3, we may find a clue to comprehend this situation. In Fig 3.3A, the powder calcined at $650^{\circ} \mathrm{C}$ has no any rigid agglomerates of the crystal grains and no distinct boundaries among those grains, so the crystal growth might not complete. Once this powder was compacted uniaxially, the crystal growth would be continued very easily at the sintering temperature $\left(920^{\circ} \mathrm{C}\right)$. From Fig 3.3B it is known that rigid agglomerates were formed and grain boundaries also became prominent in the powder calcined at $700^{\circ} \mathrm{C}$. Although these agglomerates were destroyed after pulverizing, the crystallographic anisotropy of each grain still exists, which would limit the grain growth in the compacted bulk so that the grain size in sample Co349-700 was much smaller than that in the sample Co349-650 shown in Fig 3.5. In sample Co349-750 and Co349-800, the sizes for most grains were similar to those in sample Co349-700, but both these two samples display a bimodal grain size distribution. This phenomenon can be explained by abnormal or secondary grain growth. ${ }^{[32]}$ During the final sintering process in the samples 
made from these two powders, the small, normal grains may continue to grow but the large abnormal or secondary grains grow at faster rates and grow by annihilation of surrounding normal grains. Theoretically, this type of grain growth would continue until all abnormal grains had impinged and all normal grains had been annihilated. In our work, the compacted sample was sintered at $920^{\circ} \mathrm{C}$ for a limited time period and the grain growth was constrained because of the compact pressure. As a consequence, abnormal grain growth in sample Co349-750 and Co349-800 caused the formation of huge grains and normal grain growth created smaller sizes of most grains.

Under the same pelletizing pressure and through a same sintering process, the final density only exhibited a slight increase with increasing the calcinations temperature for powder precursor. The densities of the sintered samples are 3.67 (Co349-650), 3.66 (Co349-700), 3.71 (Co349-750) and 3.84 (Co349-800) $\mathrm{g} / \mathrm{cm}$ corresponding to $82 \%, 82 \%, 83 \%$ and $86 \%$ respectively of the theoretical density. ${ }^{[24]}$ This density is not as high as those samples made by special fabrication methods, ${ }^{[3-7]}$ but still higher than the sample made by normal fabrication method using the powder produced by solid-state reaction method.

The temperature dependence of the electrical resistivity, seebeck coefficient, and power factor along the pressed surface versus temperature (T) for sintered pellets are shown in Fig 3.6a, b, and c respectively. Figure 3.6a displays the temperature dependence of electrical resistivity $(\rho)$ of the samples. In general all the samples except the sample Co349-650 show similar transport behavior with a gradually metal-insulator (MI) transition behavior around $300-400^{\circ} \mathrm{C}$, which was consistent with most research results about Co349. ${ }^{[4,7]}$ At higher measured temperature range, the electrical resistivity values show an increasing trend with the increasing calcination temperature. The lowest electrical resistivity happened on the sample Co349-650 which contributed to a higher figure of merit ZT. The Seebeck coefficient of all the samples in Fig 3.6b shows positive values over the measured temperature range, indicating a hole conduction mechanism in these compounds. For all the samples, the values of $S$ increase with increasing temperature over the measured temperature range. It is also clear that the values of $S$ for all the samples except sample Co349-800 at a certain measured temperature increase with increasing the calcinations temperature for the powder precursor. However, at higher measured temperature range the Seebeck coefficient was very close to each other for different sample. At the measured temperature $710^{\circ} \mathrm{C}$, the Seebeck coefficient value for the sample Co349-650 has is $163 \mu \mathrm{VK}^{-1}$; however, for the sample Co349-800 it reachs slightly higher value at $168 \mu \mathrm{VK}^{-1}$. As a whole, the contribution of decrease of electrical resistivity is higher than the contribution of increase of seebeck coefficient to the power factor, which results in $24 \%$ increase on the power factor for Co349-650 (0.304) compared with that for Co349-800 (0.246). The simultaneous decrease of the electrical resistivity and the Seebeck coefficient with decreasing the calcinations temperature can be explained by the increase of the carrier concentration in the system, which is consistent with the general relationship among carrier concentration, the electrical resistivity and the thermoelectric power. 


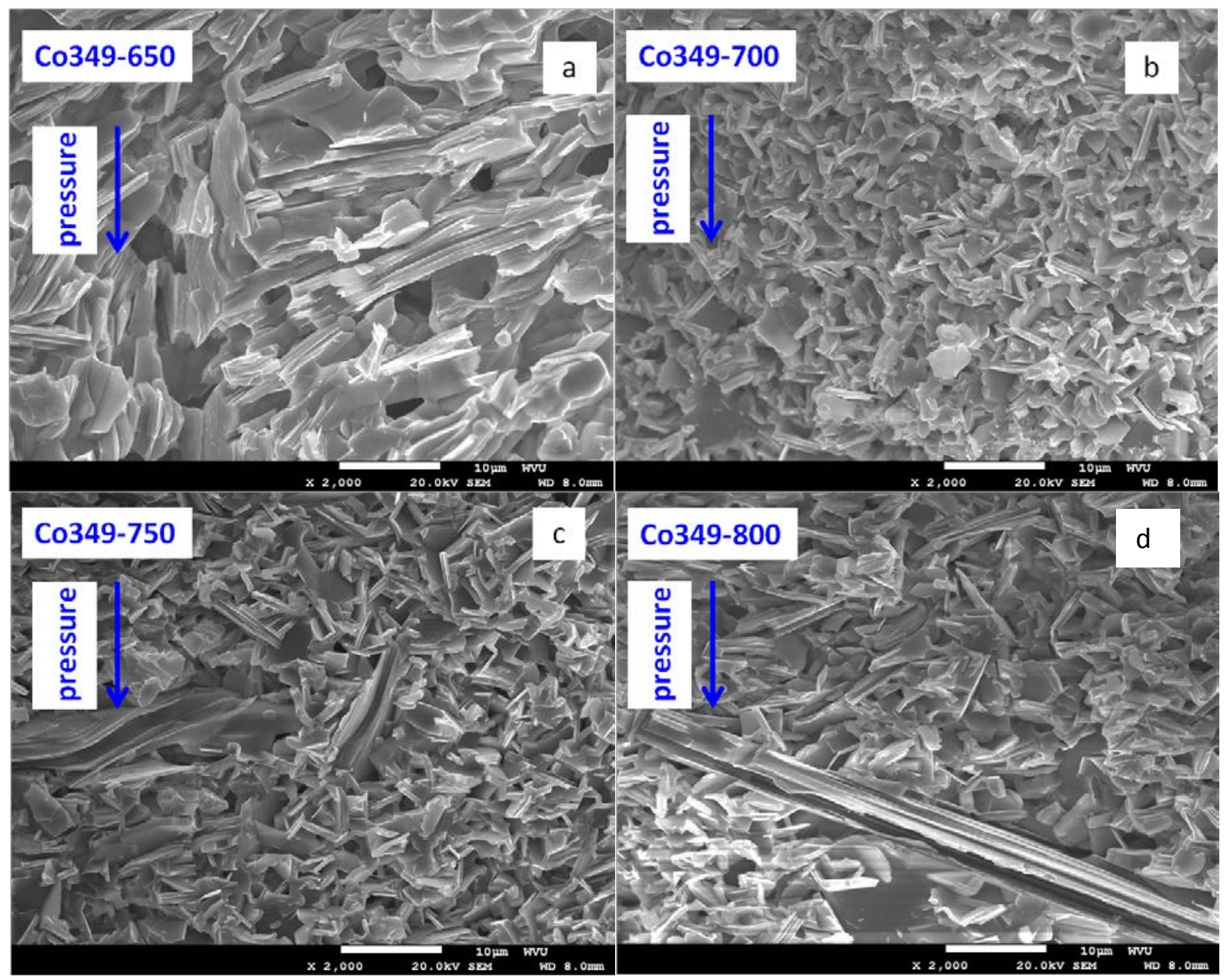

Figure 3. 5 A comparison of SEM micrographs (scale bar: $10 \mu \mathrm{m}$ ) measured on fractured surface corresponding to those sintered samples: Co349-650, Co349-700, Co349-750, and Co349-800. All the pellets were annealed at $920^{\circ} \mathrm{C}$ for $12 \mathrm{hrs}$. 

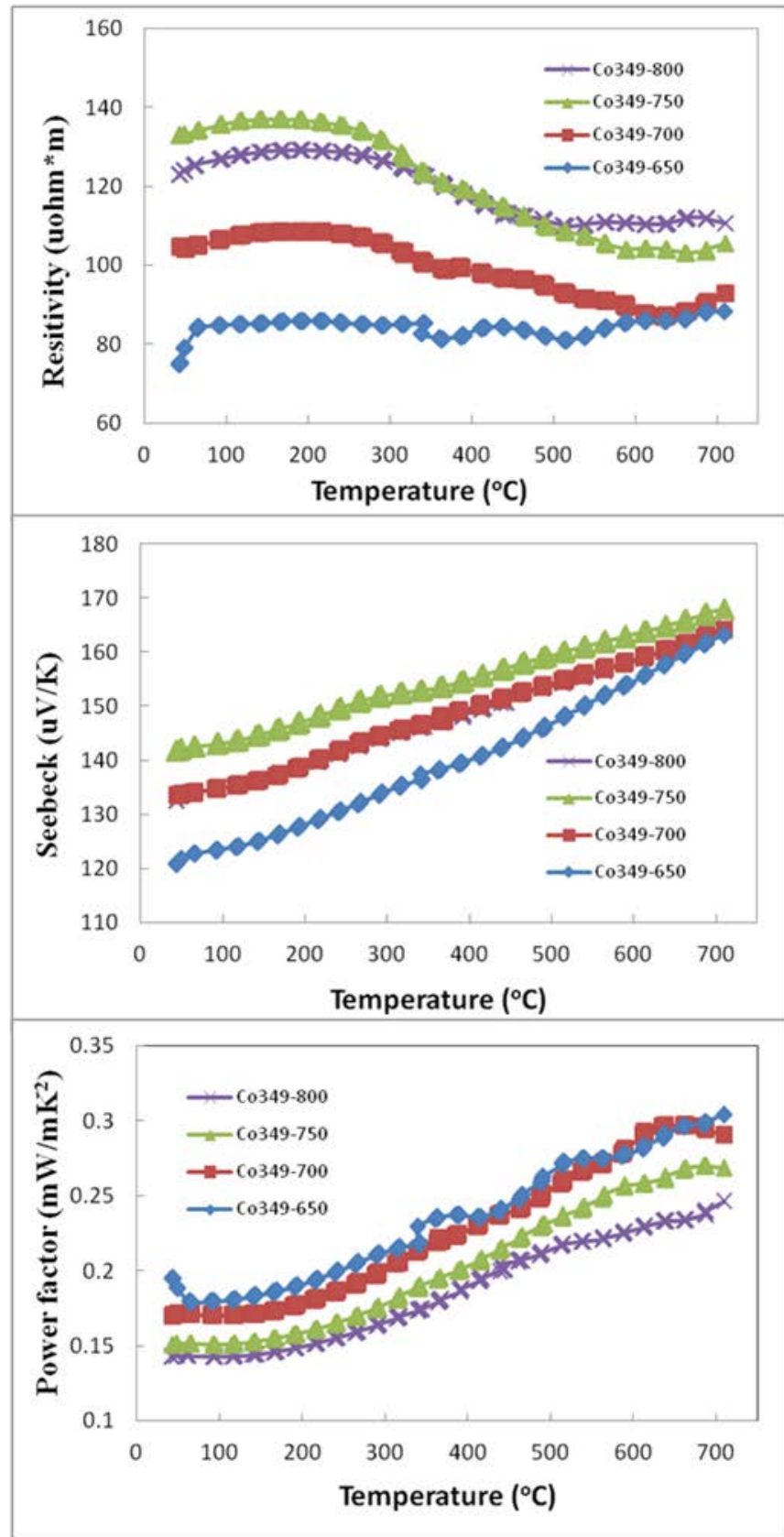

Figure 3. 6 Temperature dependence of the (a) electrical resistivity $\rho$, (b) Seebeck coefficient $S$, and (c) Power factor $S^{2} / \rho$ of Co349-650, Co349-700, Co349-750 and Co349-800. 


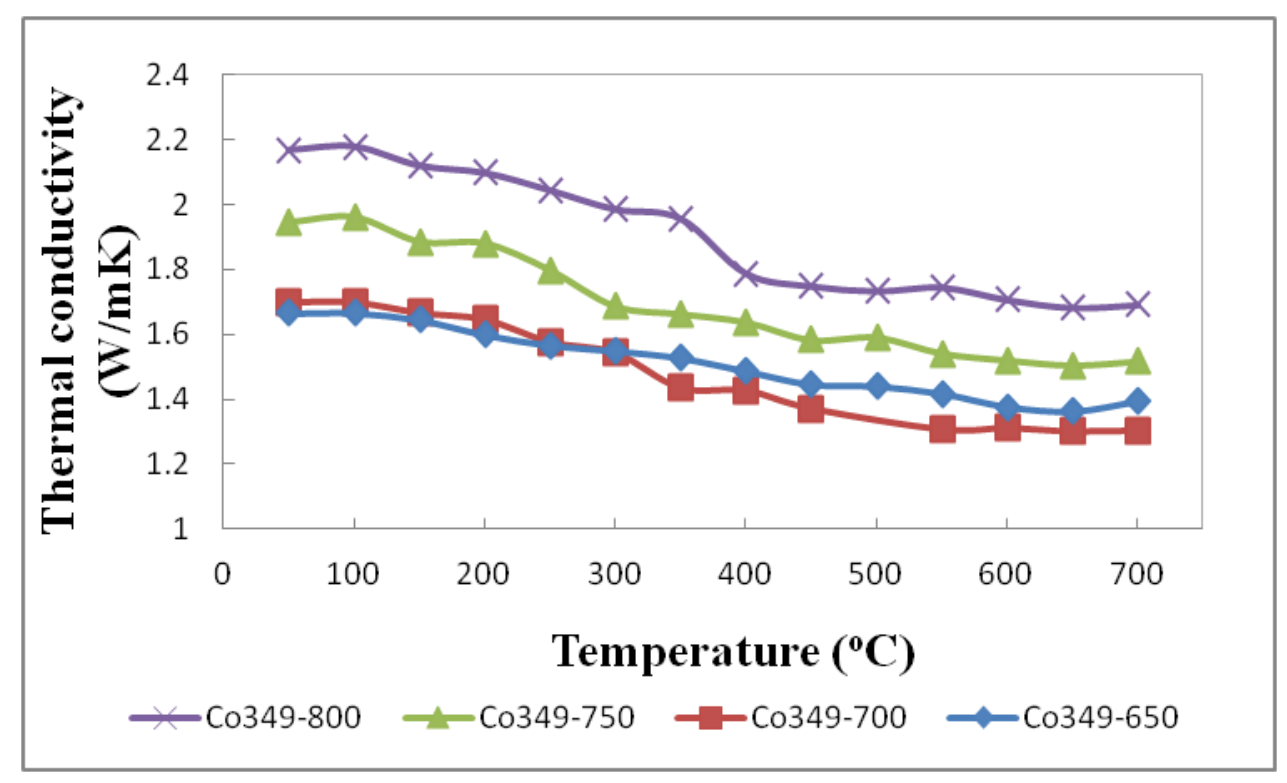

Figure 3. 7 Temperature dependence of the thermal conductivity of Co349-650, Co349-700, Co349-750 and Co349-800 along the out-of-plane (pressing) direction.

Figure 3.7 displays the thermal conductivity $\kappa$. Among those samples, at the same measured temperature the thermal conductivity of the final sintered body increase with increasing the calcinations temperature for powder precursor. The thermal conductivity $\kappa$ is related to the transfer of heat through a material, either by carrier (electrons or holes) or by quantized vibrations of the lattice, called phonons, which can represented by $\kappa_{\text {car }}$ and $\kappa_{\text {ph }}$ respectively ( $\kappa=$ $\left.\kappa_{\mathrm{car}}+\kappa_{\mathrm{ph}}\right)$. According to the Wiedemann-Franz law, the electronic term $\kappa_{\text {car }}$ is directly related to the electrical resistivity represented by the following relationship:

$$
\kappa_{\mathrm{car}}=L T / \rho=n e \mu L T
$$

where $L$ is the Lorenz factor. The Lorenz factor is usually calculated using the reduced Fermi energy, which can be estimated from the Seebeck coefficient and the Fermi-Dirac integral. Here, we use $\mathrm{L}$ with an approximate value of $2.44 \times 10^{-8} \mathrm{~W} \Omega \cdot \mathrm{K}^{-2}$. The lattice contribution $\kappa_{\mathrm{ph}}$ can be obtained by subtracting $\kappa_{\text {car }}$ from $\kappa$. The temperature dependence of $\kappa_{\mathrm{ph}}$ and $\kappa_{\mathrm{car}}$ for samples is shown in Fig 3.8. The value of $\kappa_{\mathrm{ph}}$ is much larger than that of $\kappa_{\mathrm{car}}$. With increasing the calcinations temperature for powder precursor, $\kappa_{\text {car }}$ generally decrease, which is consistent with the inverse trend of the value of electrical resistivity, as shown in Fig 3.6a. Also from equation 2, it is clearly seen that a higher electrical conductivity $\rho$ will be accompanied by a higher $\kappa_{\text {car }}$, i.e., the product of $\rho$ and $\kappa_{\text {car }}$ is essentially constant at a given temperature. However, with increasing the calcinations temperature for powder precursor, the value of $\kappa_{\mathrm{ph}}$ increased tremendously. Furthermore, with increasing the calcinations temperature the enhancement of $\kappa_{\mathrm{ph}}$ in final 
sintered body is much larger than the reduction of $\kappa_{\text {car }}$, which result in higher total thermal conductivity $\kappa$ appeared in the sample with higher calcinations temperature. From both theory and experimental results, as we mentioned before, for a pure TE material with almost fixed value of $S$ the only efficient way to increase $Z T$ is to minimize the thermal conductivity contributed by phonon scattering. Scattering phonons were attempted by using a variety of methods such as mass fluctuation scattering (a mixed crystal, in ternary and quaternary compounds), "rattling" scattering, grain-boundary scattering (due to the size of the grains), and interface scattering in thin films or multilayer systems. ${ }^{[11]}$

In our work, the difference of $\kappa_{\mathrm{ph}}$ for different samples comes from the difference of the grain boundary scattering. Smaller the grain size, lower the value of $\kappa_{\mathrm{ph}}$. As matter of fact, the grain size is the critical factor for controlling the value of $\kappa_{\mathrm{ph}}$. We can easily find a clue from SEM images in Fig 3.5, where it is clearly seen that huge grains appear in Co349-750 and Co349-800, and the number of large grains in Co349-800 is more than that in Co349-750, which makes the thermal conductivity of Co349-800 larger than that of Co349-750. Since it is hardly seen that huge grains exist in sample Co349-700, its $\kappa_{\text {ph }}$ is much lower than those of both Co349-750 and Co349-800.

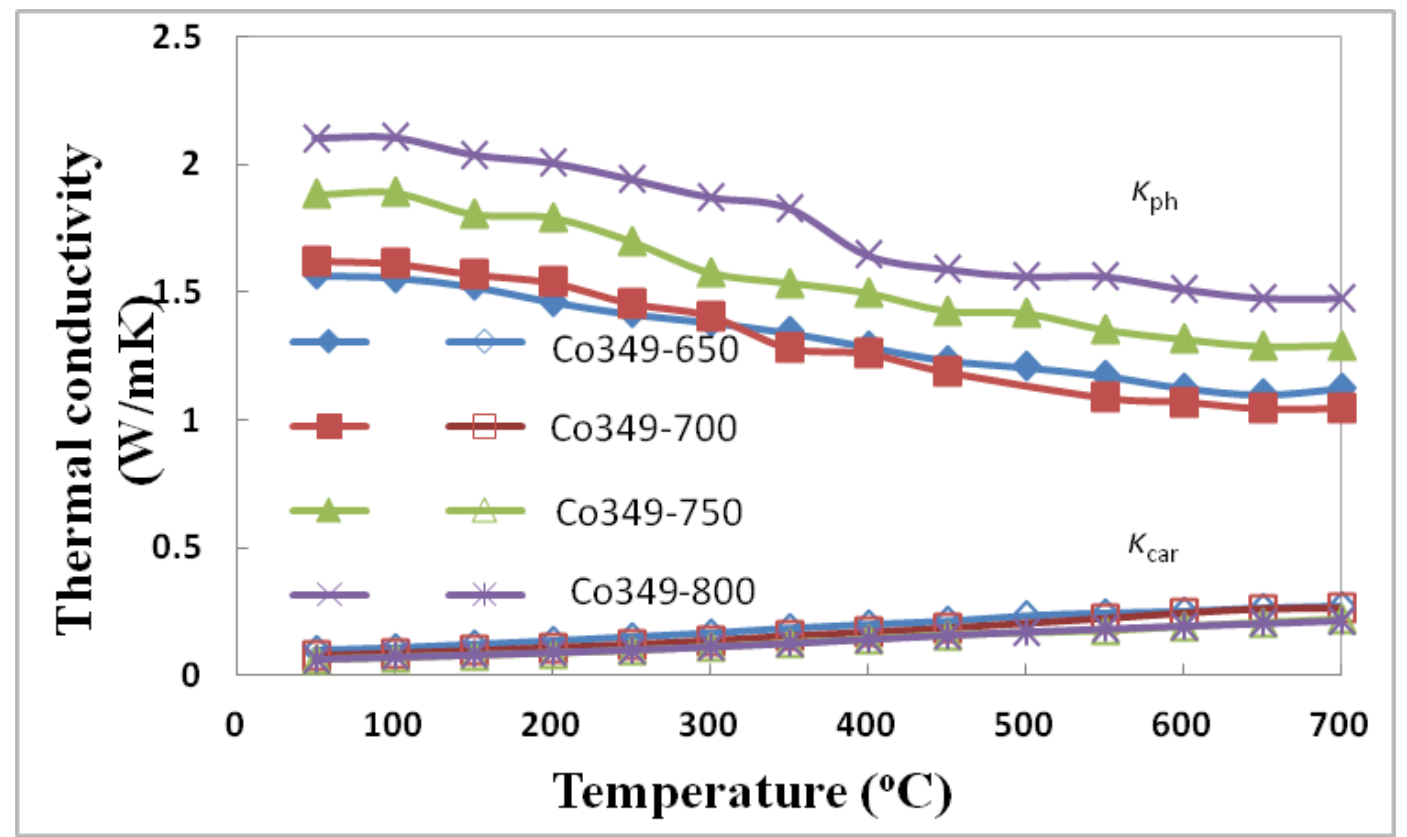

Figure 3. 8 Temperature dependence of $\kappa_{\mathrm{car}}$ and $\kappa_{\mathrm{ph}}$ for the samples prepared with the powder precursor calcined at different temperatures.

\subsection{Conclusions}


In summary, the calcinations temperature of powder precursor has a critical effect on the thermoelectric properties in final sintered Co349 bulk materials. The electrical resistivity and the Seebeck coefficient along in-plane direction are increased with increasing the calcinations temperature for powder, however, when the powder was calcined at higher temperature $800^{\circ} \mathrm{C}$, the Seebeck coefficient are decreased by almost overlapping with that of Co349-700. All the samples except the sample Co349-650 show similar transport behavior with a gradually metalinsulator (MI) transition behavior around $300-400^{\circ} \mathrm{C}$. Thermal conductivity along out-of-plane direction was increased with increasing the calcinations temperature. The power factors for Co349-650 and Co349-700 are the highest and almost same with each other. 


\section{Chapter 4: Anisotropic Thermoelectric Properties in a Misfit- layered Calcium Cobaltite Affected by Pelletizing Pressure}

\subsection{Objective}

The calcined $\mathrm{Ca}_{3} \mathrm{Co}_{4} \mathrm{O}_{9}$ (Co349) powder precursors were pressed under different pressure such as $0.25 \mathrm{GPa}, 0.5 \mathrm{GPa}$, and $1.0 \mathrm{GPa}$. Then all the samples were sintered at $920^{\circ} \mathrm{C}$ for $24 \mathrm{hrs}$. The Seebeck and electrical resistivity were measured only along in-plane direction, however thermal conductivities along both in-plane and out-of-plane directions were obtained. Combine the analysis of the microstructure and thermoelectric properties, this study was proposed to study the anisotropy of Co349 under different pressing conditions and determine the proper condition to obtain the highest figure of merit.

This study is a compensation for the optimization technique of ceramic processing. Both calcinations condition as we studied in chapter 3 and pelletizing condition are thought to be most important factors for determining the thermoelectric properties of final sintered ceramics.

\subsection{Introduction}

The anisotropy of misfit-layered cobaltite Co349 was always the focus on which the researchers usually discussed. According to Masset et al., ${ }^{[24]}$ a small piece of single crystal Co349 shows much higher electrical resistivity of in-plane $\left(\rho_{\mathrm{ab}}\right)$ than that of out-of-plane $\left(\rho_{\mathrm{c}}\right)$. The results suggest that preferentially oriented bulk polycrystals would exhibit a similar behavior. Furthermore, according to Wiedemann-Franz law, the carrier term $\kappa_{\text {car }}$ of thermal conductivity is directly related to the electrical resistivity as shown in equation 3.1, which makes it easily to be understood that low electrical resistivity will always be accompanied by high thermal conductivity.

As a matter of fact, Moon et al. ${ }^{[14]}$ found that in the preferentially oriented polycrystal of $\left(\mathrm{Ca}_{2.7} \mathrm{Bi}_{0.3}\right)\left(\mathrm{Co}_{3.7} \mathrm{Cu}_{0.3}\right) \mathrm{O}_{9+\delta}$, the electrical conductivity along in-plane direction was about four times larger than that along out-of-plane direction, while the Seebeck coefficients were similar. However, the thermal conductivity was highest along the in-plane; the figure-of-merit along the in-plane was significantly enhanced solely due to the enhanced electrical conductivity.

Moreover, the anisotropy in thermoelectric properties essentially exists in highly grain-aligned Co349 ceramic. The ratio of figure-of-merit between in-plane and out-of-plane will be determined by the ratio of electrical resistivity, thermal conductivity and Seebeck coefficient between these two directions. As Zhou et al. ${ }^{[5]}$ already discussed, $\left(\mathrm{Ca}_{2.7} \mathrm{Sr}_{0.2} \mathrm{La}_{0.1}\right)\left(\mathrm{Co}_{3.9} \mathrm{Cu}_{0.1}\right) \mathrm{O}_{9}$ (CSLCCO) sample show the figure-of-merit with 0.41 by supposing isotropic thermal conductivity, but only 0.19 by using the same $\kappa_{\text {in-plane }} / \kappa_{\text {out-of-plane }}$ ratio reported by Moon $e t$ al. ${ }^{[14]}$ 
In order to obtain highly grain-aligned and densified Co349 sample, some special fabrication methods have been used, ${ }^{[3-7]}$ such as Hot-Pressing (HP), Cold high pressure pressing, Magnetic Alignment (MA) and Spark Plasma Sintering (SPS) process. In those researches, the isotropic thermal conductivity was supposed to calculate the figure-of-merit along in-plane direction. However, as we know the further study of the effect of anisotropy of thermal conductivity on the value of figure-of-merit is still needed.

The anisotropy of thermoelectric properties was considered to depend on the degree of grain orientation, ${ }^{[14]}$ i.e. texturing behavior. The degree of out-of-plane orientation is calculated by the Lotgering method, ${ }^{[33]}$ which is one of the ways to evaluate the degree of orientation for various materials. ${ }^{[5,34]}$ The Lotgering factor, $f$, is defined in the following equations:

$$
\begin{gathered}
f=\frac{P-P_{0}}{1-P_{0}} \\
P=\frac{\sum I_{(00 l)}}{\sum I_{(h k l)}}
\end{gathered}
$$

where $P$ is the sum of the relative intensities for all $(00 l)$ diffractions divided by the sum of all the relative intensities of $(h \mathrm{kl})$ diffractions in the samples. $P_{0}$ is an equivalent parameter for a randomly oriented specimen. The Lotgering factor, $f$, varies, ranging from 0 for an utterly nonoriented specimen to 1 for a completely oriented one. Owing to the limited penetration of X-rays in materials, the Lotgering factor represents the extent of grain alignment in a layer of $50 \mu \mathrm{m}$ underneath the surface.

The objective of this study is to fabricate pellets of Co349 using cold-press under different pressure and then to investigate the effect of pressure on the development of crystal texture and electrical properties of sintered Co349. The anisotropy of thermal conductivity was investigated by measuring the transport properties along the in-plane and the out-of-plane respectively.

\subsection{Experimental procedures}

The sol-gel preparation is similar with that described in chapter 3. After the sol-gel mixture was charred and combusted into powder at $500^{\circ} \mathrm{C}$ on the hot stage, the obtained powder was completely ground and calcined at $800^{\circ} \mathrm{C}$ for $4 \mathrm{~h}$ with oxygen flow. The appropriate amount of calcined powder was loaded into a dry pressing die with a 1 inch central cylindrical opening diameter and is immediately pressed under $0.25 \mathrm{GPa}, 0.5 \mathrm{GPa}$, or $1 \mathrm{GPa}$ to obtain pellets, which will be represented by Co349-0.25, Co349-0.5 or Co349-1.0, respectively. Under each pressing condition, three pellets were produced. Two of them were thinner with thickness at $\sim 2 \mathrm{~mm}$ and the third one is thicker with thickness at $\sim 10 \mathrm{~mm}$. The obtained pellets were sintered at $920^{\circ} \mathrm{C}$ for $24 \mathrm{~h}$ in tube furnace with oxygen flow. 
The final sintered bodies with different processing pressure at 0.25GPa, 0.5GPa, or 1.0GPa were represented by Co349-0.25, Co349-0.5, and Co349-1.0, respectively. The phase identification of the obtained samples was made by X-ray diffraction (Bruker D8 Discovery) with $\mathrm{Cu} \mathrm{K}_{\alpha}$ radiation at room temperature. All the samples were measured on the pressed surface. Both the particle morphology for powder and the surface micrograph for bulk samples were investigated by a field emission scanning electron microscope (FE-SEM; JEOL JSM-7600F).

For certain pressure of pellet fabrication, one thinner pellet was cut into $2 \mathrm{~mm} \times 2 \mathrm{~mm} \times 8$ $\mathrm{mm}$ bars from the pellet discs for the Seebeck coefficient $(S)$ and electrical resistivity $(\rho)$ measurements. Both $S$ and $\rho$ for all the pellets were simultaneously measured by the standard four-probe dc method (LINSEIS, LSR-3 Seebeck) in helium atmosphere over the temperature range from room temperature to $700^{\circ} \mathrm{C}$. The measurements were carried out along in-plane direction. Another thinner one and the thick pellet were used for thermal diffusivity and heat capacity measurement along in-plane direction and along out-of-plane direction. The thicker pellet was cut into $2 \mathrm{~mm} \times 10 \mathrm{~mm} \times 13 \mathrm{~mm}$ bars for thermal property measurement. The thermal diffusivity $\alpha(\mathrm{T})$ and specific heat $C_{\mathrm{p}}(\mathrm{T})$ measurement were conducted by the laser flash method (LINSEIS, LFA 1000) simultaneously. For $C_{\mathrm{p}}(\mathrm{T})$ measurement we also compare the result with that measured by a differential scanning calorimetry (DSC) method. The results for $C_{\mathrm{p}}(\mathrm{T})$ measured by two different methods are almost same. Then we choose the laser flash method since it can be done simultaneously with $\alpha(\mathrm{T})$ measurement. The thermal conductivity $\kappa$ of the sample was calculated from $\alpha(\mathrm{T}), C_{\mathrm{p}}(\mathrm{T})$, and density $D$ using $\kappa=\alpha C_{\mathrm{p}} D$.

\subsection{Results and discussion}

SEM images for the pressed surface morphologies of Co349 pressed under different pressure are shown in Fig 4.1. Images a, b, and c are representing sample Co349-0.25, Co349-0.5, and Co349-1.0 respectively. All the samples were sintered at $920^{\circ} \mathrm{C}$ for 24 hrs with oxygen flow. From Fig 4.1, it is clearly seen that with increasing pelletizing pressure, the connection among grains becomes more tightly. Furthermore, with increasing the pelletizing pressure, the grain size tends to change from uniform to bimodal distribution. Especially when under the higher pressure such as 1.0 GPa, on the pressed surface almost half area is covered by grains with size around 2 $\mu \mathrm{m}$, however the other half area is dominated by larger grains with size above $5 \mu \mathrm{m}$, some of which are even larger than $10 \mu \mathrm{m}$. On the surface of sample with lower pelletizing pressure, although larger grains exist on the surface, the amount of smaller grains is much lower and the compactness among the grains is not as high as that in sample with higher pelletizing pressure. 

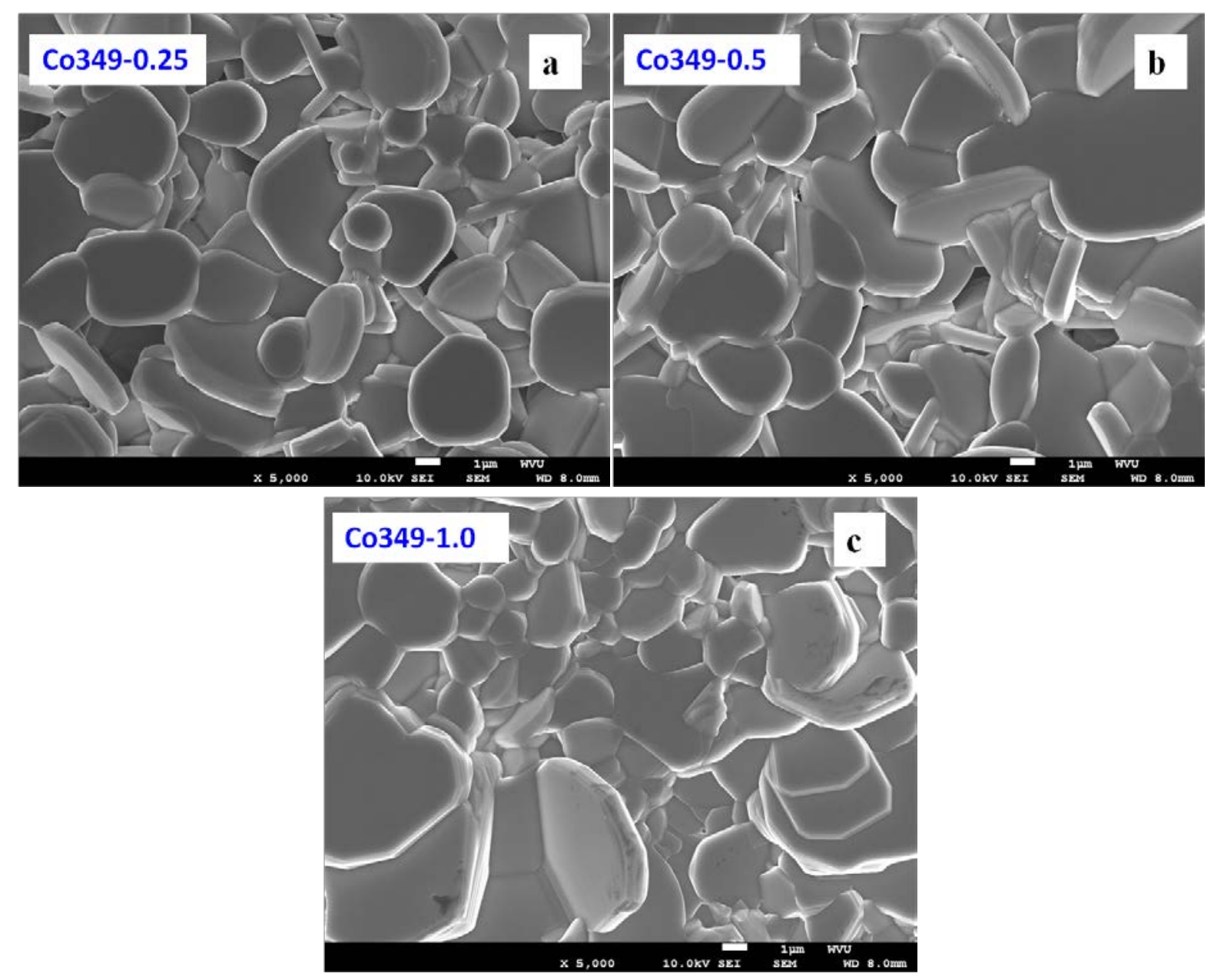

Figure 4. 1 SEM surface morphology of Co349 sintered at $920^{\circ} \mathrm{C}$ for $24 \mathrm{~h}$ with different pelletizing condition: (a) 0.25GPa (Co349-0.25), (b) 0.5GPa (Co349-0.5), and (c) 1.0GPa (Co349-1.0)

SEM images for the fractured surface morphologies of Co349 with different pelletizing pressure are shown in Fig 4.2. Fig 4.2 a, b, and c represent sample Co349-0.25, Co349-0.5, and Co349-1.0, respectively. Different from in pressed surface, the grain size and size distribution in fractured surface have no apparent differences among these three samples. Compared with those samples made by special fabrication methods, ${ }^{[3-7]}$ our samples which were made under relatively low pelletizing pressures have no strong texture.

To further clarify the texture characteristic of the samples, XRD measurements for the pressed surface of the specimens were conducted as shown in Fig 4.3. Generally, the degree of crystallographic anisotropy of a material can be evaluated in terms of the Lotgering factor $f$, where $p_{0}=p$ for a crystallographic isotropic (randomly oriented) sample that can be calculated from the peak data of reference, i.e. $p_{0}=0.449$ for powder Co349. ${ }^{[35]}$ The XRD results are shown in Fig 4.3. Although the XRD profile for the pressed surface of these three samples 

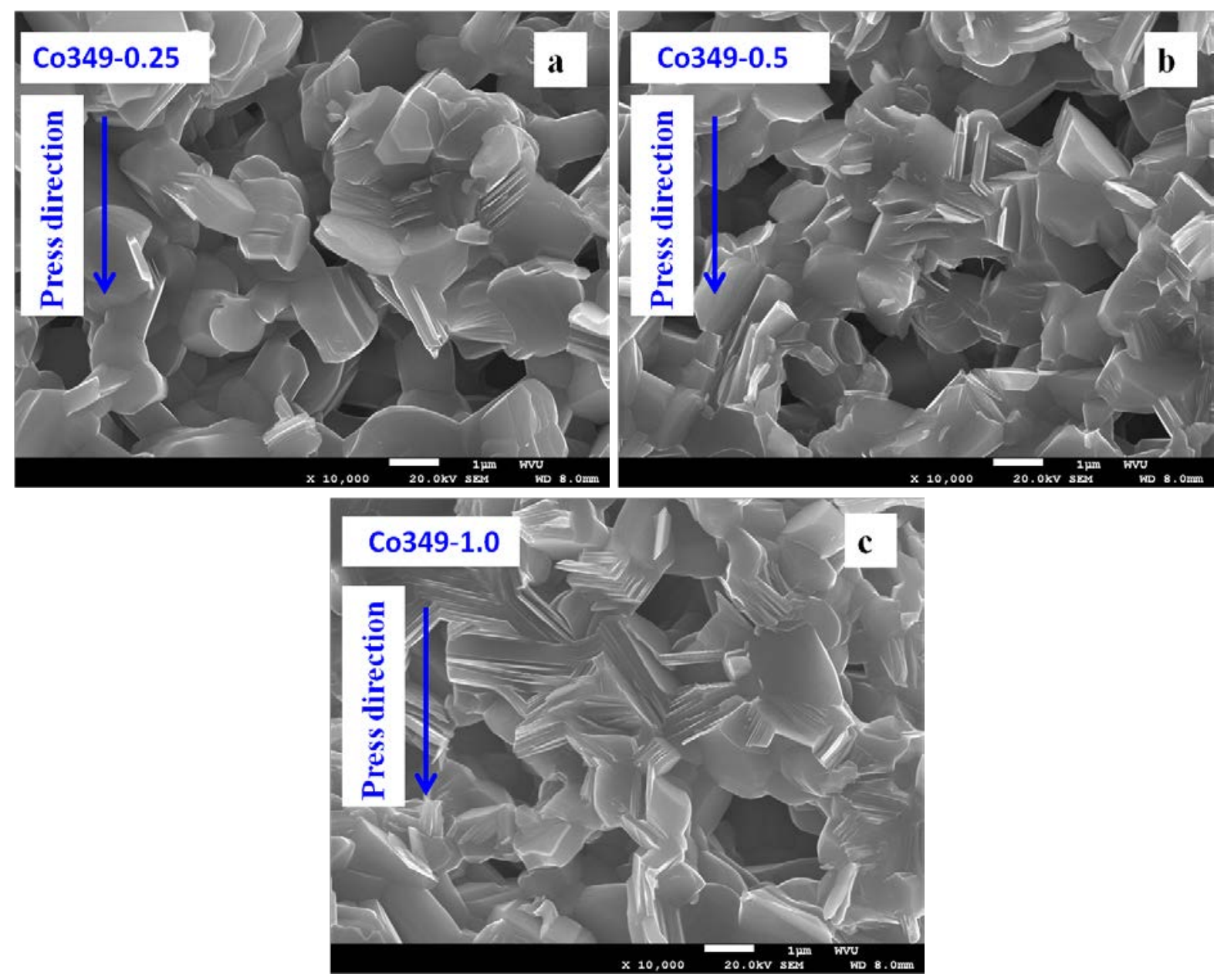

Figure 4. 2 SEM fractured surface morphology of Co349 sintered at $920^{\circ} \mathrm{C}$ for $24 \mathrm{~h}$ with different pelletizing condition: (a) 0.25GPa (Co349-0.25), (b) 0.5GPa (Co349-0.5), and (c) 1.0GPa (Co349-1.0).

exhibit strong diffraction peaks from (00l) planes, the other diffraction peaks from other crystal planes, such as 201 and 202 planes, are also very strong. The Lotgering factors, which are calculated using $p_{0}=0.45$ as the reference, ${ }^{[35]}$ are $0.42,0.53$, and 0.45 for sample Co3491.0GPa, Co349-0.5GPa, and Co349-0.25GPa, respectively. At the same time, we also prepared our reference sample, which was pelletized under extremely low pressure and annealed at the same condition as those three samples. The Lotgering factors are $0.22,0.37,0.27$ correspondingly, by using the value of $p_{0}=0.59$, which is calculated from our reference sample. The value $f$ of $(00 l)$ planes for these three samples is not as high as that for the cold high-pressure pelletized sample $(f=0.9)^{[35]}$, suggesting that the crystallographic anisotropy of the sample is not as high as those samples made by special fabrication methods. ${ }^{[3-7]}$ This is consistent with the microstructures on the fractured surface as shown in Fig 4.2 and confirmed by the thermal conductivity measurements along in-plane and out-of-plane directions, which will be discussed later. 


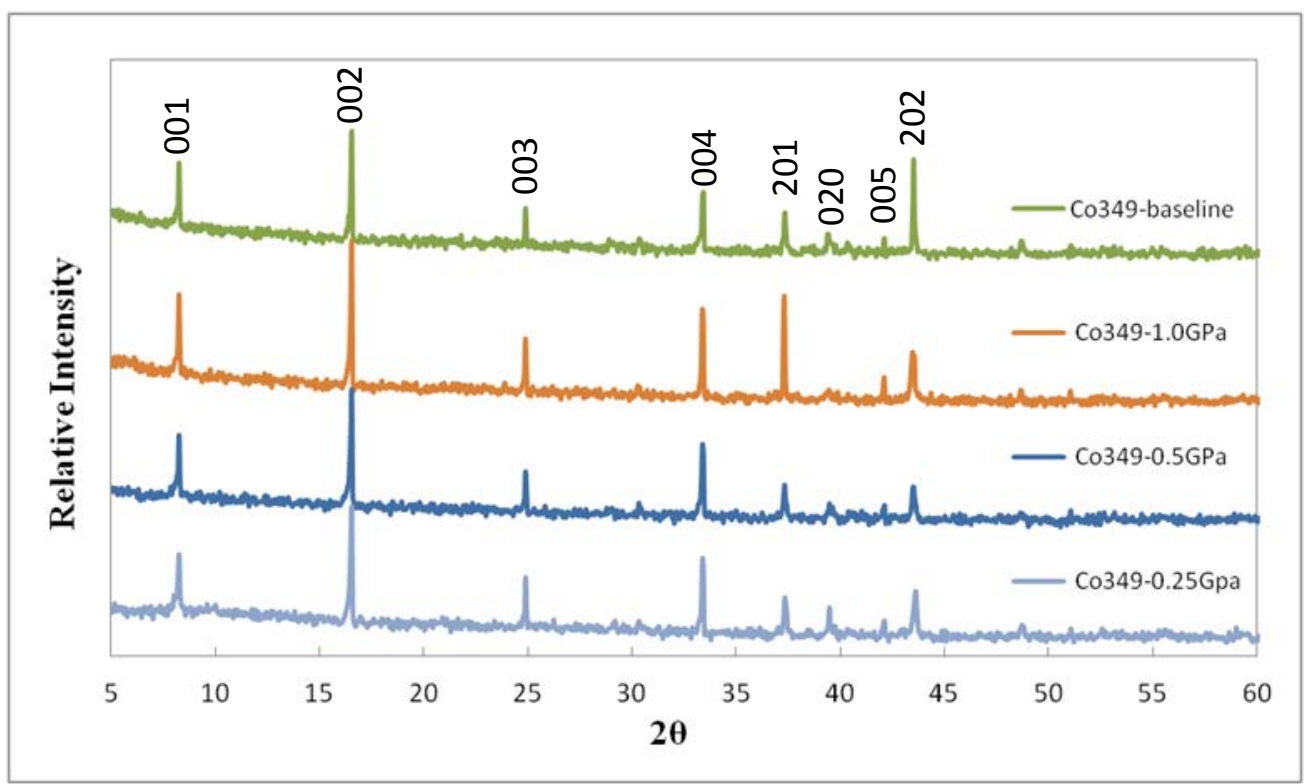

Figure 4. 3 X-ray diffraction pattern of $\mathrm{Ca}_{3} \mathrm{Co}_{4} \mathrm{O}_{9}$ pellet pressed under different pressure and powder baseline (the pressed pellet and baseline sample was sintered at $920^{\circ} \mathrm{C}$ for $24 \mathrm{~h}$ ).

As we can see from the Lotgering factors listed in Table 4.1, with increasing pelletizing pressure the degree of grain-alignment in sample does increase with increasing pressure from $0.25 \mathrm{GPa}$ to $0.5 \mathrm{GPa}$, but decreases when the pelletizing pressure reaches $1.0 \mathrm{GPa}$. When we discuss the dependence of texturing behaviors on pelletizing pressure, all the other factors were ignored since all the samples were made starting from sample powder and same annealing process except the pelletizing pressure. However, these factors may affect each other. When the powders were pelletized under different pressure, the green bodies may have different texture extent caused by pressure. After the green bodies were annealed at higher temperature, the texture extent would be increased. This was proved by the following experiment.

In order to confirm the dependence of texturing behavior on the annealing process, we prepared another pellet and measured it by XRD before annealing and after annealing process. The pellet was pelletized under the pressure even lower than $0.25 \mathrm{GPa}$, which was represented as Co349-baseline. The $\mathrm{p}$ value calculated from XRD results shows a value of 0.42 before annealing and after annealing procedure. The result indicates that the extent of texture does increase after annealing procedure, which is consistent with the results done by Tsai et al. They have investigated the texturing behaviors and kinetics of $\mathrm{NaCo}_{2} \mathrm{O}_{4-\delta}$ with increasing annealing process duration and showed that the texturing processing was dominated by self-diffusion mechanism under annealing temperature. ${ }^{[36]}$ 
Table 4. 1 Lotgering factors for Samples pelletizing under different pressure

\begin{tabular}{|c|c|c|c|c|c|c|c|c|c|c|c|}
\hline he & \multicolumn{8}{|c|}{ Integral of diffraction peak } & & & \\
\hline samp & 001 & 002 & 003 & 004 & 005 & 201 & 020 & 202 & $\mathrm{p}$ & $f_{\mathrm{a}}$ & $f_{\mathrm{b}}$ \\
\hline $1.0 \mathrm{GPa}$ & 92 & 143 & 62 & 110 & 28 & 103 & 92 & 7 & 0.68 & 0.22 & 0.42 \\
\hline $0.5 \mathrm{GPa}$ & 50 & 131 & 43 & 95 & 19 & 43 & 71 & 6 & 0.74 & 0.37 & 0.53 \\
\hline $0.25 \mathrm{GPa}$ & 48 & 166 & 49 & 100 & 18 & 52 & 85 & 23 & 0.70 & 0.27 & 0.45 \\
\hline Baseline $^{\mathrm{a}}$ & 46 & 101 & 35 & 58 & 11 & 50 & 96 & 29 & 0.59 & & \\
\hline Baseline $^{\mathrm{b}}$ & & & & & & & & & 0.45 & & \\
\hline
\end{tabular}

Notes: a. The XRD diffraction pattern obtained from the sample with extremely low pelletizing pressure, which is corresponding to Lotgering factor $f_{\mathrm{a}}$.

b. The XRD baseline is from the reference ${ }^{[35]}$, which is corresponding to Lotgering factor $f_{\mathrm{b}}$.

Figure 4.4 presents the temperature dependence of (a) Seebeck coefficient $S$, (b) electrical resistivity $\rho$, and (c) power factor $S^{2} / \rho$ of Co349-1.0GPa, Co349-0.5GPa and Co349-0.25GPa. From Fig 4.3a we can see the Seebeck coefficients for all three samples are increased with increasing the measurement temperature and the value for Co349-1GPa is always greater than those for the other two samples, except when temperature reaches higher than $600^{\circ} \mathrm{C}$, the value for the former sample is lower than those for the latter two samples. During the whole temperature range, the speed of $S$ increasing for Co349-1.0GPa is much lower than that for the other samples. Furthermore, at room temperature the value of $S$ for Co349-1GPa that is around $140 \mu \mathrm{V} / \mathrm{K}$, is much higher than those for the other two samples, which are both close to 125 $\mu \mathrm{V} / \mathrm{K}$.

Figure 4.4b displays the temperature dependence of electrical resistivity $(\rho)$ of the samples. Both Co349-0.25GPa and Co349-0.5GPa show an increasing trend with the increasing measurement temperature. However, Co349-1.0GPa has a gradually metal-insulator (MI) transition behavior around $300-400^{\circ} \mathrm{C}$, which was similar with most research results about $\mathrm{Ca}_{3} \mathrm{Co}_{4} \mathrm{O}_{9} .{ }^{[4,7]}$ At room temperature, the electrical resistivity has a sequence for these three samples: Co349-0.25GPa > Co349-1.0GPa > Co349-0.5GPa, which is consistent with the sequence of Lotgering factors for these samples: Co349-0.25GPa < Co349-1.0GPa < Co3490.5GPa. Higher the Lotgering factor, higher the texturing extent and then lower the electrical resistivity. 


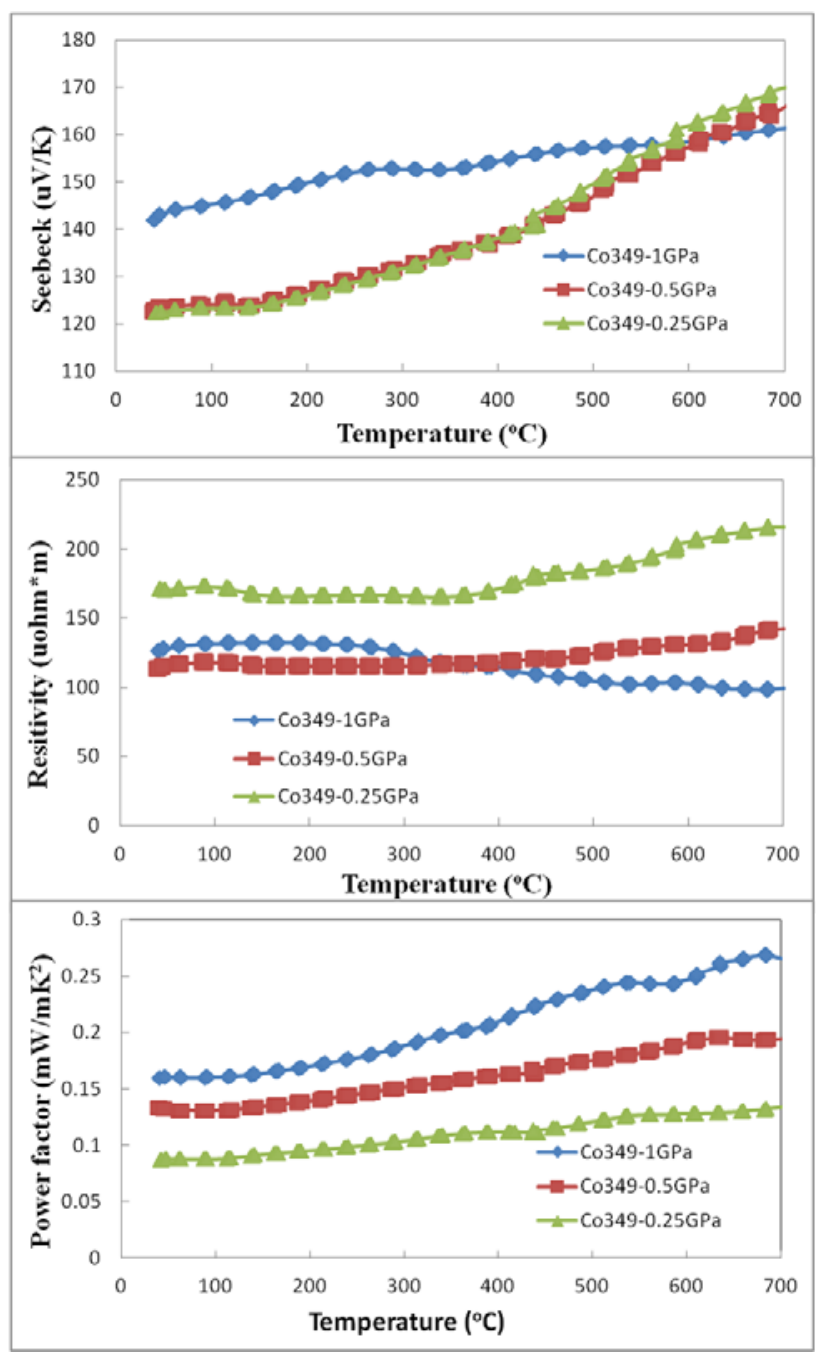

Figure 4. 4 Temperature dependence of the (a) Seebeck coefficient $S$, (b) electrical resistivity $\rho$, and (c) Power factor $S^{2} / \rho$ of Co349-1.0GPa, Co349-0.5GPa and Co349-0.25GPa.

As a result, the highest powder factor exists in sample Co349-1.0GPa, which reaches 0.27 $\mathrm{mW} / \mathrm{mK}^{2}$. This value is comparable with that reported for the sample made by cold highpressure fabrication method ${ }^{[4]}$, but lower than the value for the sample made by SPS method, ${ }^{[5]}$ which shows a value around $0.3 \mathrm{~mW} / \mathrm{mK}^{2}$. However, the thermal conductivity for Co349-1.0GPa is much lower than that for sample made by SPS method. We will discuss the details later.

There is one aspect we need emphasize that although the higher pressure will not necessarily cause a higher texturing extent, the packing density of the pellets is higher, which may cause the difference in Seebeck coefficient and electrical resistivity.

Figure 4.5 displays the temperature dependence of (a) thermal conductivity $\kappa$, (b) figure-ofmerit ZT of Co349-1.0GPa, Co349-0.5GPa, and Co349-0.25GPa. In order to compare the ratio of 


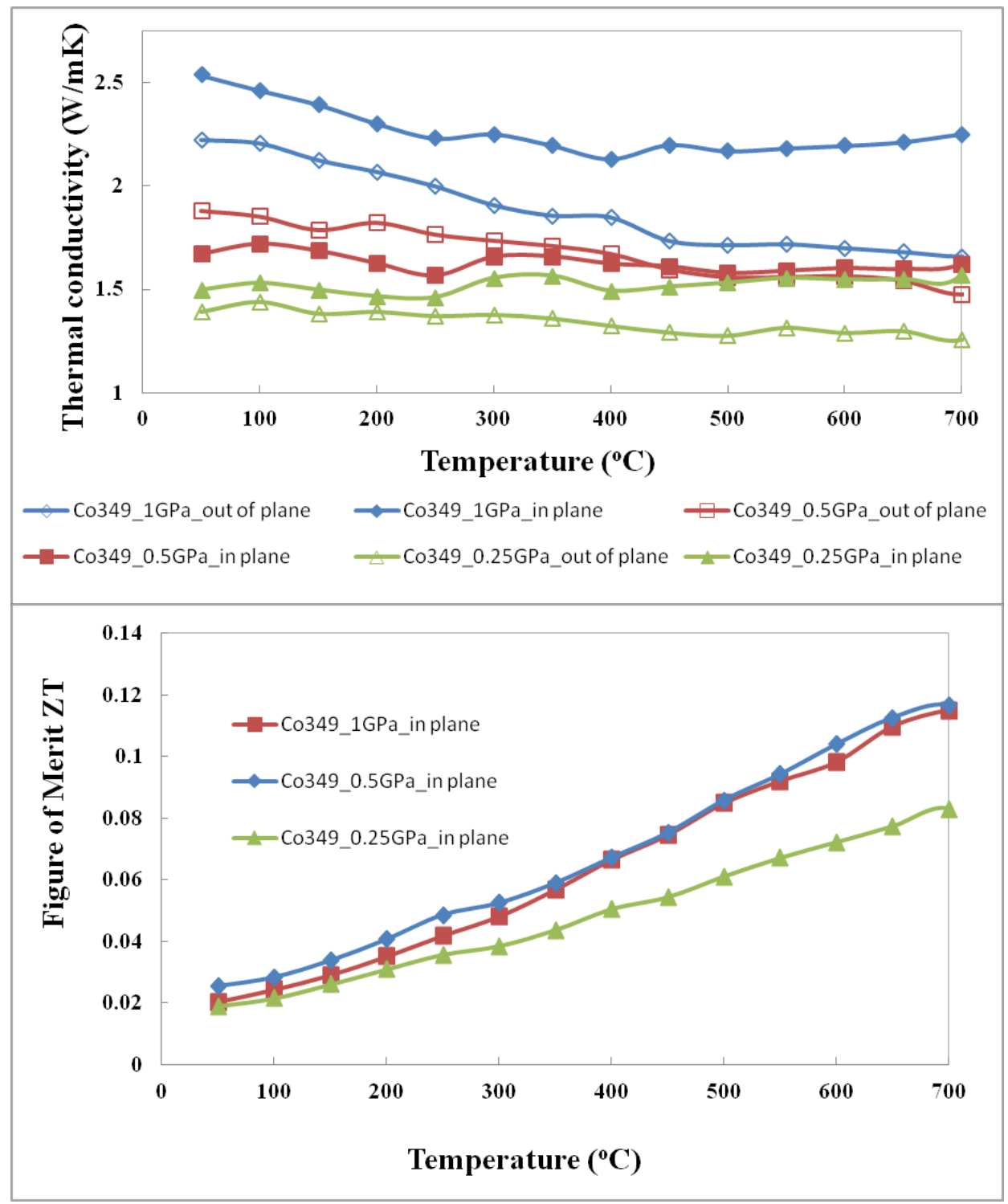

Figure 4. 5 Temperature dependence of the (a) thermal conductivity, (b) ZT of Co349-1GPa, Co3490.5GPa and Co349-0.25GPa. Empty symbols represent the value across in-plane; filled symbols represent the values along in-plane.

thermal conductivity along in-plane and out-of-plane directions among the samples with different pelletizing pressure, the thermal conductivities were measured along both in-plane and out-of-plane directions. The result is displayed in Fig 4.5a. Since the Seebeck coefficient and electrical resistivity were measured along only in-plane direction, we can only obtain the figureof-merit ZT with $S, \rho$ and $\kappa$ along the same direction, i.e. along in-plane direciton. Then this direction is called as the "in plane" direction and, for ZT and $\kappa$, the samples are named such as Co349_0.5GPa_in plane. The other direction is called as the "out of plane” direction since $S$ and 
$\rho$ were measured along in-plane direction but $\kappa$ was measured along out-of-plane direction, and then the samples are named such as Co349_0.5GPa_out of plane.

For sample Co349-1.0GPa and Co349-0.25GPa, the ratio of $\kappa$ along two perpendicular directions very big. The value of $\kappa$ along in-plane direction is much higher than that along out-ofplane direction. However, the ratio is much lower than that reported for highly grain-aligned samples, ${ }^{[14]}$ where the thermal conductivity along in-plane was more than twice as large as that along out-of-plane direction, because our sample have no such high texturing extent. If only considering the texture extent, we should conclude that the Co349-0.5GPa has the highest ratio of thermal conductivity along those two directions; however, this sample has no apparent difference in thermal conductivity along two directions especially under high measurement temperature. This indicates the texturing extent is not the crucial factor causing the difference in thermal conductivity and figure-of-merit along in-plane and out-of-plane directions.

Figure 4.5b displays the comparison of figure-of-merit ZT for different samples. If ZT was calculated with the thermal conductivity measured along out-of-plane direction, the value is much higher than that calculated using the thermal conductivity along in-plane direction. Although the ratio of ZT for two directions for sample Co349-1.0GPa is larger than that for sample Co349-0.5GPa, the value of ZT for Co349_1.0GPa_in plane is almost same as that for Co349_0.5GPa_in plane. This indicates the pelletizing pressure does not improve the figure-ofmerit from $0.5 \mathrm{GPa}$ to $1.0 \mathrm{GPa}$. This result will cause a doubt whether highly grain-alignment can improve the value of figure-of-merit, although the anisotropic thermoelectric properties exist in highly grain-aligned sample.

\subsection{Conclusions}

Polycrystalline Co349 materials were prepared using the sol-gel reaction technique. The calcinations temperature for powder precursor was chosen at $800^{\circ} \mathrm{C}$. The dependence of texturing behavior and thermoelectric properties of Co349 on pelletizing pressure were investigated quantitatively. Scanning electron microscopy was used for texture observation. Lotergering factors calculated from XRD results further proved that the texturing extent in our samples was not as large as that in those samples fabricated by special methods. Although increasing the pelletizing pressure, the electrical resistivity was decreased along in-plane direction, but the figure-of-merit was not improved since the thermal conductivity was increased along the same direction. The anisotropy of thermal conductivity decreased and then increased with pelletizing pressure from 0.25GPa to 1.0GPa.

In this chapter, only one temperature was chosen for calcinations. As we already know from chapter 3, the samples with different calcinations temperature have different thermoelectric properties. Therefore, the trend of dependence of thermoelectric properties on the pelletizing pressure may also different. In the future work, we will do some research on this point. 


\section{Chapter 5: $\mathrm{Ca}_{3} \mathrm{Co}_{4} \mathrm{O}_{9}$ Bulk Ceramics and Their Thermoelectric Properties and Nanostructure Evolution upon High Temperature Annealing}

\subsection{Objective}

Since $\mathrm{Ca}_{3} \mathrm{Co}_{4} \mathrm{O}_{9}$ will be decomposed above $926^{\circ} \mathrm{C}$ as indicated in $\mathrm{CaO}-\mathrm{CoO}$ phase diagram in air atmosphere, it is worthy to study the thermoelectric properties and crystal structure of $\mathrm{Ca}_{3} \mathrm{Co}_{4} \mathrm{O}_{9}$ annealed in oxygen flow at the temperature above $926^{\circ} \mathrm{C}$.

The $\mathrm{Ca}_{3} \mathrm{Co}_{4} \mathrm{O}_{9}$ green bodies was sintered at $920^{\circ} \mathrm{C}$ (baseline) for $12 \mathrm{~h}$ first, then some samples directly were cooled down to room temperature (as baseline) but the others were annealed at $1000^{\circ} \mathrm{C}$ for another $12 \mathrm{~h}$ and then cooled down to room temperature (annealed). Both the thermoelectric properties and microstructure were analyzed.

This study was proposed to using High Resolution Transmission Electron Microscope (HRTEM) equipped with Energy Dispersive X-ray Spectroscopy (EDS) to examine the structure and chemistry from micron to atomic levels.

\subsection{Introduction}

Thermoelectric oxide materials, ${ }^{[1-2,24,37-40]}$ such as newly developed $\mathrm{Ca}_{3} \mathrm{Co}_{4} \mathrm{O}_{9}$, ${ }^{[41-45]}$ are particularly promising for on-board heavy-duty vehicle applications and high temperature waste heat harvesting from the power plant, because they are lightweight, cheaply produced, highly efficient and stable at high temperatures in air, and non-toxic. For practical applications, TE materials must first posses a high conversion efficiency, which is measured by the dimensionless figure-of-merit, ZT. ZT is defined as $S^{2} \sigma T / \kappa$, where $S$ is the Seebeck coefficient, $\sigma$ is the electrical conductivity, $\kappa$ is the thermal conductivity, and $T$ is the absolute temperature. Materials with larger $S$, higher $\sigma$, and lower $\kappa$ are desired for application. The efficiency reaches about $10 \%$ at $Z T=1$, and hence $Z T \geq 1$ is generally accepted as a criterion for practical applications. ${ }^{[46]}$ The $\mathrm{Ca}_{3} \mathrm{Co}_{4} \mathrm{O}_{9}$ single crystal shows good TE behavior with a ZT extrapolated to be 0.8 at $973 \mathrm{~K}\left(700^{\circ} \mathrm{C}\right),{ }^{[2,42]}$ and is highly stable in air up to $1199 \mathrm{~K}\left(926^{\circ} \mathrm{C}\right) .{ }^{[19]}$ The current challenge for developing oxide TE materials is to improve the conversion efficiency of polycrystalline $\mathrm{Ca}_{3} \mathrm{Co}_{4} \mathrm{O}_{9}$, which is currently low compared with conventional TE materials. ${ }^{[12]}$

The extraordinary thermoelectric performance of $\mathrm{Ca}_{3} \mathrm{Co}_{4} \mathrm{O}_{9}$ is attributed to its layered structure. $\mathrm{Ca}_{3} \mathrm{Co}_{4} \mathrm{O}_{9}$ contains layers of single $\mathrm{CdI}_{2}$-type $\mathrm{CoO}_{2}$ and triple rock salt-type $\mathrm{Ca}_{2} \mathrm{CoO}_{3}$ subunits that are alternately stacked in the $c$-direction with a misfit relationship. In the $\mathrm{Ca}_{3} \mathrm{Co}_{4} \mathrm{O}_{9}$, $\mathrm{CoO}_{2}$ sheets, possessing a strongly correlated electron system serve as electronic transport layers, while $\mathrm{Ca}_{2} \mathrm{CoO}_{3}$ layers serve as phonon scattering regions to give low thermal conductivity. The misfit relationship between the $\mathrm{CoO}_{2}$ layer and $\mathrm{Ca}_{2} \mathrm{CoCo}_{3}$ layer leads to an incommensurate 
structure with the subunits sharing the same $a, c$ and $\beta$ parameters but different $b$ lattice parameters $\left(b_{1} / b_{2}=1.61\right){ }^{[24]}$ The subunit of $\mathrm{CoO}_{2}$ from $\mathrm{Ca}_{3} \mathrm{Co}_{4} \mathrm{O}_{9}$ phase are also shared by other Ca-Co-O cobaltite, including $\mathrm{Ca}_{2} \mathrm{Co}_{2} \mathrm{O}_{5},{ }^{[47-48]} \mathrm{CaCo}_{2} \mathrm{O}_{4},{ }^{[49]}$ and $\mathrm{Ca}_{3} \mathrm{Co}_{2} \mathrm{O}_{6}{ }^{[19,}{ }^{26]}$ In the pseudobinary Ca-Co-O system, stable phases have been synthesized for $\mathrm{Ca} / \mathrm{Co}$ ranging between 1.5 and 0.75 of $\mathrm{Ca}_{x} \mathrm{CoO}_{2},{ }^{[2,49]} \mathrm{Ca}_{3} \mathrm{Co}_{2} \mathrm{O}_{6},{ }^{[26]} \mathrm{Ca}_{2} \mathrm{Co}_{2} \mathrm{O}_{5}{ }^{[21,}{ }^{50-51]}$ and $\mathrm{Ca}_{3} \mathrm{Co}_{4} \mathrm{O}_{9} .{ }^{[24]}$ Among those similar cobaltite phases, $\mathrm{Ca}_{3} \mathrm{Co}_{4} \mathrm{O}_{9}$ exhibits the best TE behavior. During the synthesis of the $\mathrm{Ca}_{3} \mathrm{Co}_{4} \mathrm{O}_{9}$ phase, it is considered that the formation of the above-mentioned phases of calcium cobalt oxide are dependent on sintering temperature, and the $\mathrm{Ca}_{3} \mathrm{Co}_{4} \mathrm{O}_{9}$ phase is stable up to $926^{\circ} \mathrm{C}$ and then decomposes into the $\mathrm{Ca}_{3} \mathrm{Co}_{2} \mathrm{O}_{6}$ phase, which is stable up to $1026^{\circ} \mathrm{C} .{ }^{[20]}$ For practical applications, it is essential to understand the thermal stabilities of sintered $\mathrm{Ca}_{3} \mathrm{Co}_{4} \mathrm{O}_{9}$ materials in air, the formation of other cobaltite Ca-Co-O phases, and their property and structure evolution upon long term high temperature annealing and over-heating above the reported decomposition temperature of $1199 \mathrm{~K}$.

The present work reports on the thermoelectric performance and nanostructure of polycrystalline $\mathrm{Ca}_{3} \mathrm{Co}_{4} \mathrm{O}_{9}$ samples, synthesized using the chemical sol-gel route. In particular, the thermoelectric properties and nanostructure evolution were studied for a $\mathrm{Ca}_{3} \mathrm{Co}_{4} \mathrm{O}_{9}$ sample annealed at $1000^{\circ} \mathrm{C}$, which is over the $\mathrm{Ca}_{3} \mathrm{Co}_{4} \mathrm{O}_{9}$ decomposition temperature of $926^{\circ} \mathrm{C}$ as indicated in the $\mathrm{CaO}-\mathrm{CoO}$ phase diagram. ${ }^{[19]}$

\subsection{Experimental procedure}

The $\mathrm{Ca}_{3} \mathrm{Co}_{4} \mathrm{O}_{9}$ precursor powders with nominal composition were prepared using the same procedure as described in chapter 3 . Then the precursor was calcined at $650^{\circ} \mathrm{C}$ in a tube furnace for $4 \mathrm{~h}$ with oxygen flow to form precursor powders. The calcined powders were then regrounded and uniaxially pressed into pellets under a pressure of $0.93 \mathrm{GPa}$ at room temperature. The baseline pellets were sintered at $920^{\circ} \mathrm{C}$ for 12 hours. The annealed pellets were first sintered at $920^{\circ} \mathrm{C}$ for 12 hours, and then further annealed at $1000^{\circ} \mathrm{C}$ for another 12 hours. Both sintering and annealing procedures were performed in a tube furnace with oxygen flow.

The absolute Seebeck coefficient and electrical resistivity of the pellets were measured in Helium gas with low pressure from $50^{\circ} \mathrm{C}$ to $750^{\circ} \mathrm{C}$ using the Linseis LSR-1100. For both the baseline and annealed samples, the electrical properties and Seebeck coefficients were measured along the in plane direction, i.e., along the pressed plane of the pellets. The thermal diffusivity $\lambda$ was measured from $50^{\circ} \mathrm{C}$ to $750^{\circ} \mathrm{C}$ using the Linseis Laser Flash Analyzer (LFA) 1200. The specific heat $C_{\mathrm{p}}$ were also examined using the LFA, by flashing methods and comparing the test samples to a reference sample with known specific heat and tested under the same condition. For both the baseline and the annealed sample, the thermal properties including the specific heat and thermal diffusivity were measured in the directions parallel (in plane) and perpendicular to the pressed plane (out of plane) of the pellets. X-ray Diffraction (XRD), Scanning Electron Microscope (SEM), and High Resolution Transmission Electron Microscope (HRTEM) equipped with Energy Dispersive X-ray Spectroscopy (EDS) were used to examine the structure 
and chemistry from micron to atomic levels. TEM samples were prepared by mechanical polishing and ion milling in a liquid-nitrogen cooled holder. Electron diffraction, diffraction contrast, high-resolution TEM imaging and chemistry analysis were performed in a JEM-2100 operated at $200 \mathrm{kV}$.

\subsection{Results and Discussion}

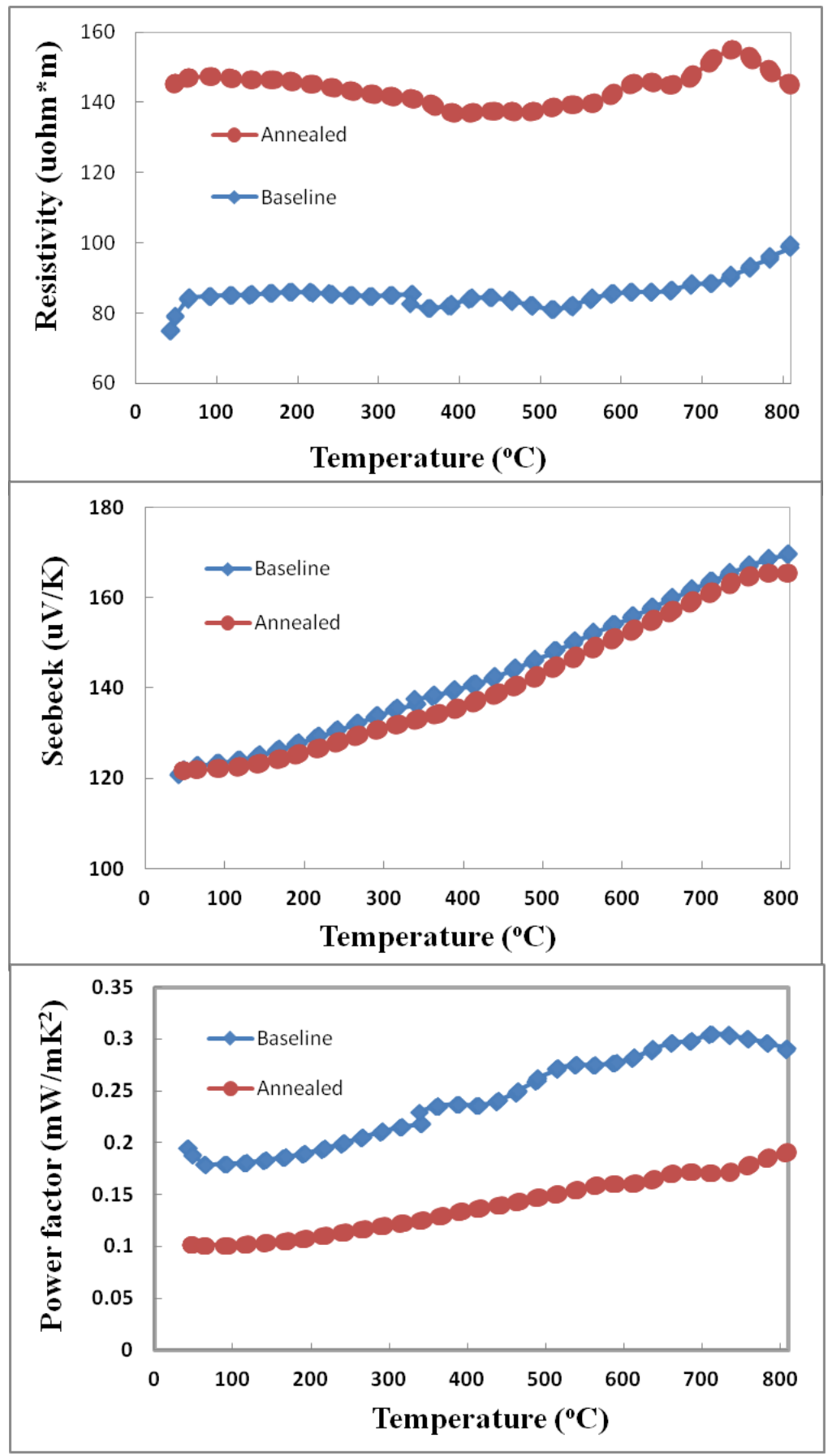


Figure 5. 1 Temperature dependence of the (a) Seebeck coefficient $S$, (b) electrical resistivity $\rho$, and (c) Power factor $S^{2} / \rho$ of Co349-baseline and Co349-annealed.

\subsubsection{Thermoelectric performance of the baseline and annealed samples}

\section{(1) Electrical properties}

As shown in Fig 5.1(a), the baseline sample has an absolute Seebeck coefficient $S$ of 120.7 $\mu \mathrm{VK}^{-1}$ at $50^{\circ} \mathrm{C}$. The $S$ increases gradually with the increase of temperature and reaches 169.7 $\mu \mathrm{V} / \mathrm{K}$ at $808^{\circ} \mathrm{C}$. The annealed sample shows a slightly lower $S$ compared with the baseline sample from $50^{\circ} \mathrm{C}$ to $808^{\circ} \mathrm{C}$. Fig 5.1 (a) indicates that annealing at the high temperature of $1000^{\circ} \mathrm{C}$ has little impact on the absolute Seebeck coefficient. The electrical resistivity from both the baseline and annealed pellets are shown in Fig 1 (b). The baseline sample exhibits low electrical resistivity of $74.7 \mu \Omega \cdot \mathrm{m}$ at $50^{\circ} \mathrm{C}$, and has very weak temperature dependence as the temperature rises. The resistivity reaches $99.7 \mu \Omega \cdot \mathrm{m}$ at $808^{\circ} \mathrm{C}$. For the sample annealed at $1000^{\circ} \mathrm{C}$, no significant change of resistivity was observed corresponding to the temperature change, in which electrical resistivity is $145 \mu \Omega \cdot \mathrm{m}$ at $50^{\circ} \mathrm{C}$ and $144.8 \mu \Omega \cdot \mathrm{m}$ at $808^{\circ} \mathrm{C}$, respectively. As shown in Fig. 1 (c), the baseline sample possesses the high power factor of 190 $\mu \mathrm{Wm}{ }^{-1} \mathrm{~K}^{-2}$ at $50^{\circ} \mathrm{C}$, and $290 \mu \mathrm{Wm}^{-1} \mathrm{~K}^{-2}$ at $808^{\circ} \mathrm{C}$. The highest power factor is $310 \mu \mathrm{Wm}^{-1} \mathrm{~K}^{-2}$ at $713^{\circ} \mathrm{C}$. Resulting from the comparable Seebeck coefficient and significantly increased resistivity upon high temperature annealing, the power factor of the annealed sample is obviously lower than that from the baseline sample. The power factor of the annealed sample shows increases as temperature increases and reaches only $190 \mu \mathrm{Wm}^{-1} \mathrm{~K}^{-2}$ at $808^{\circ} \mathrm{C}$.

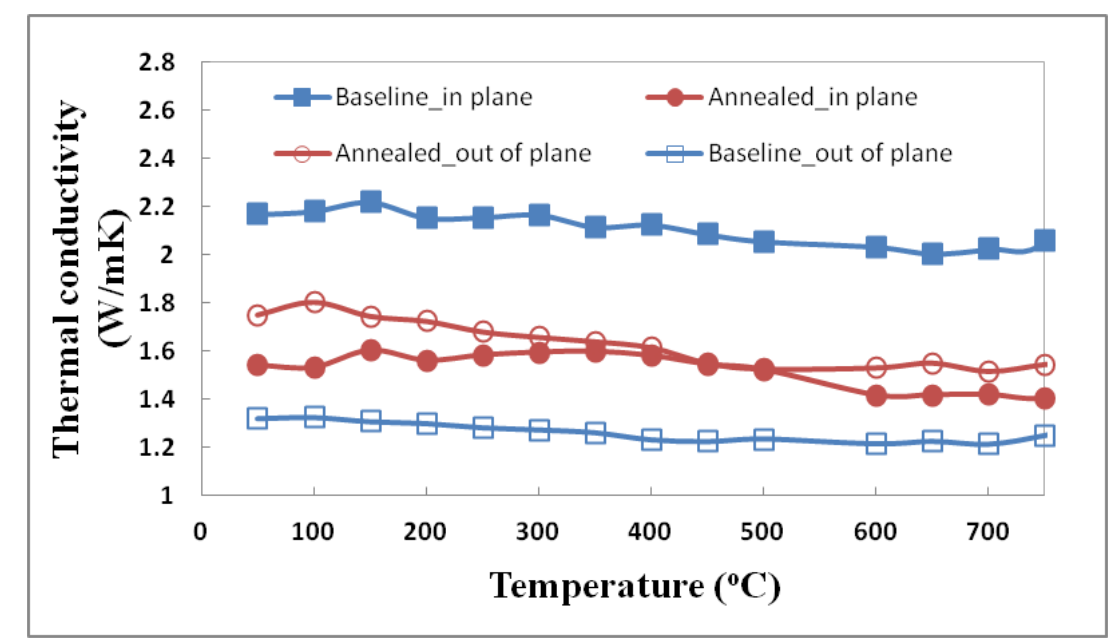

Figure 5. 2 Temperature dependence of the thermal conductivity $\kappa$. Empty symbols represent the value along out-of-plane; filled symbols represent the values along in-plane.

\section{(2) Thermal conductivity}


Thermal conductivity $\boldsymbol{\kappa}$ from parallel and perpendicular to the pressed plane of the pellets were obtained. Strong anisotropy of thermal conductivity was measured in the baseline sample. The baseline sample shows the higher $\kappa$ of $2.19 \mathrm{Wm}^{-1} \mathrm{~K}^{-1}$ at $50^{\circ} \mathrm{C}$ and $2.06 \mathrm{Wm}^{-1} \mathrm{~K}^{-1}$ at $750^{\circ} \mathrm{C}$ along the pressed plane. By contrast, the baseline sample shows the low $\kappa$ of $1.32 \mathrm{Wm}^{-1} \mathrm{~K}^{-1}$ at $50^{\circ} \mathrm{C}$ and $1.4 \mathrm{Wm}^{-1} \mathrm{~K}^{-1}$ at $750^{\circ} \mathrm{C}$ in the direction perpendicular to the pressed plane. Differing from the baseline sample, the annealed samples seem to have no strong anisotropy. As indicated in Fig 5.2, after annealing, the thermal conductivities measured parallel to the pressed plane show a only slight difference and is around $1.57 \mathrm{Wm}^{-1} \mathrm{~K}^{-1}$ at $750^{\circ} \mathrm{C}$.

\section{(3) Figure of Merit ZT}

The $Z T$ value is calculated using equation $Z T=S^{2} \sigma T / \kappa$ where $\mathrm{S}, \sigma$ and $\kappa$ are all measured for the direction parallel to the pressed plane. The plots of $Z T$ as a function of temperature for both the baseline and annealed pellets are shown in Fig 5.3. For the baseline pellets, within the examined temperature range, a $Z T$ of 0.15 is obtained at $750^{\circ} \mathrm{C}$. For the annealed samples, the $Z T$ values increase along with the increase of temperature, showing the highest of 0.13 at $750^{\circ} \mathrm{C}$.

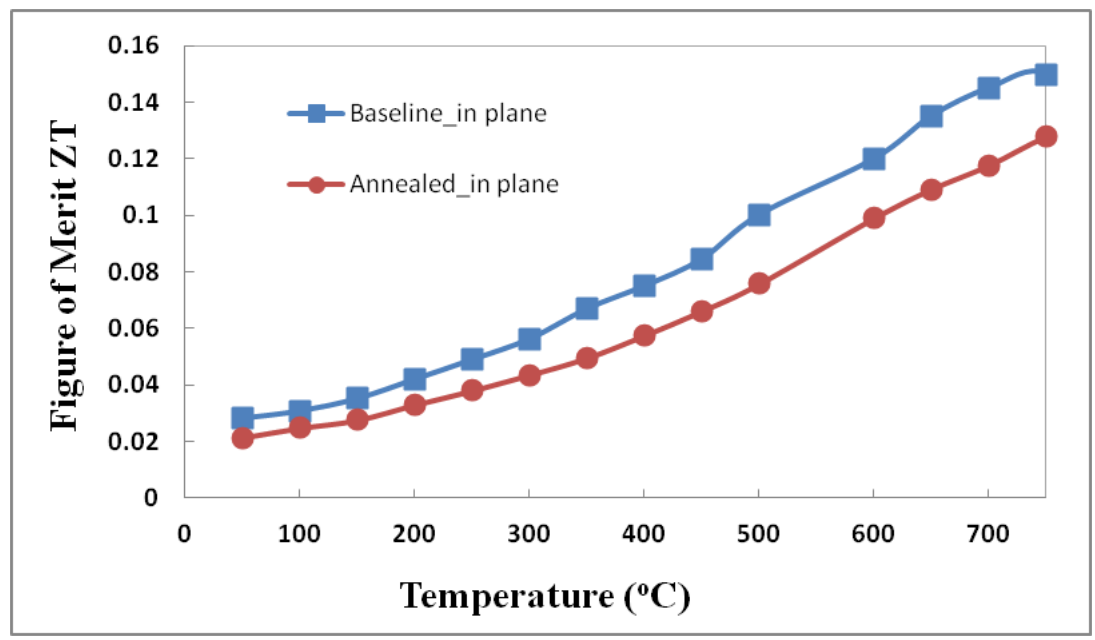

Figure 5. 3 Temperature dependence of ZT for Co349-Baseline and Co349-Annealed samples.

\subsubsection{Microstructure Characterization using XRD and SEM}

Under the cold pressing with the pressure of $0.93 \mathrm{GPa}$, the apparent density of the samples are $3.67 \mathrm{~g} / \mathrm{cm}^{3}, 3.63 \mathrm{~g} / \mathrm{cm}^{3}$ for the baseline and annealed sample respectively. XRD taken from the pressed surfaces are shown in Fig 5.4, indicating both the baseline and annealed samples are with the dominating $\mathrm{Ca}_{3} \mathrm{Co}_{4} \mathrm{O}_{9}$ phase (JCPDS 21-139) and very tiny amount of $\mathrm{Co}_{3} \mathrm{O}_{4}$. The grain morphology of the pressed surfaces of both the baseline and annealed samples is shown in Fig 5.5(a) and Fig 5.5(b), respectively. In the pressed plane of the baseline pellet, the crystals are 
plate-shaped with an average grain size in the planar dimension of $\sim 3 \mu \mathrm{m}$. By contrast, the annealed sample presents the grains with an average size of $0.5 \mu \mathrm{m}$ along the plate thickness and $\sim 3 \mu \mathrm{m}$ along the planar direction. As evident in the SEM image shown in Fig 5.5, the $\mathrm{Ca}_{3} \mathrm{Co}_{4} \mathrm{O}_{9}$ grain from the pressed surface of the baseline and the annealed sample are having the different crystal orientation. Such grain orientation change indicates possible re-crystallization occurred during the annealing process.

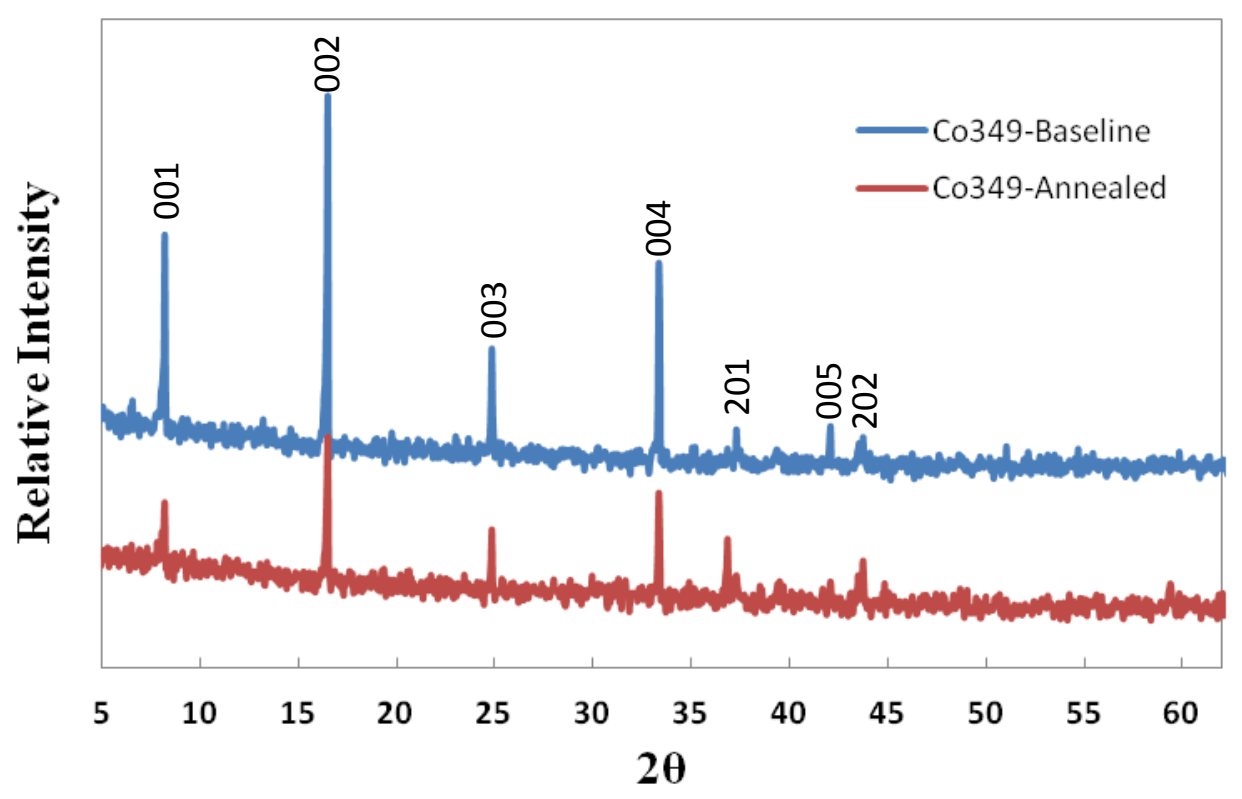

Figure 5. 4 XRD patterns for Co349-Baseline and Co349-Annealed samples.

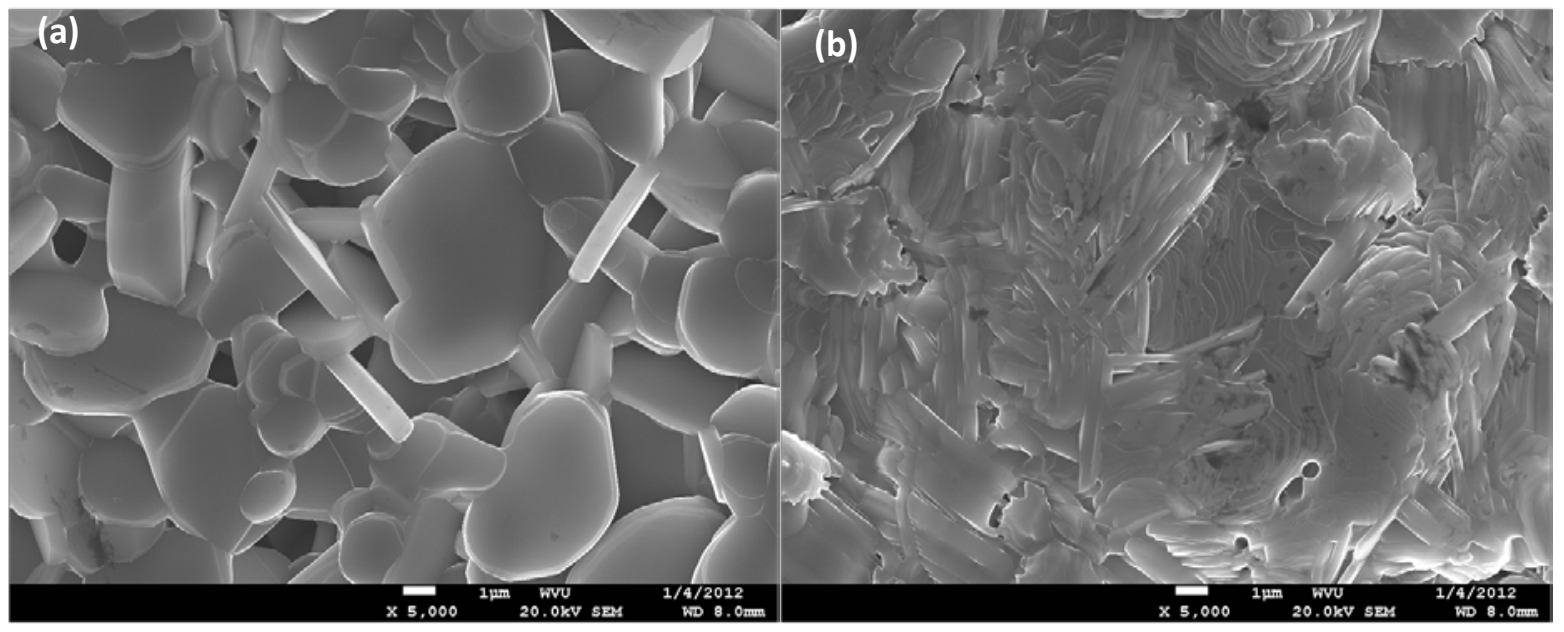

Figure 5. 5 SEM images for Co349-Baseline (a) and Co349-Annealed (b) samples. 


\subsubsection{Nanostructure and Chemistry Analysis Using TEM}

(1). Baseline sample. TEM samples were prepared to image the planes perpendicular to the pressed plane. The TEM image in Fig 5.6 (a) clearly shows that the morphology of the micron sized crystal "grains" shown in the SEM image (shown in Fig 5.5a) consist of crystal bundles with different orientations. Within each of the bundles, there is contrast from nano-lamellas. The electron diffraction patterns in Fig 5.6 (b) and (c) were taken from the different nanolamellas and show that the nano-lamellas are from the $\mathrm{Ca}_{3} \mathrm{Co}_{4} \mathrm{O}_{9}$ phase with different orientations including twinning. The longer dimension of the nano-lamellas is perpendicular to the $c$-direction of monoclinic $\mathrm{Ca}_{3} \mathrm{Co}_{4} \mathrm{O}_{9}$ phase, and micron-sized. All of the nano-lamellas in the same "bundle" have the same direction of $c$-axis, but possess different orientations in the $a b$ plane. In other words, the nano-lamellas $\mathrm{Ca}_{3} \mathrm{Co}_{4} \mathrm{O}_{9}$ within one bundle possesses a fiber texture and shares the same $c$-direction. The magnified view of the nano-lamella within one crystal bundles are shown in Fig 5.6 (d). Those nano-lamellas are with the width of 5-50 nm. One of the typical HRTEM images taken from neighboring nano-lamellas with different orientations is shown in Fig 5.7. It can be clearly seen from Fig 5.7 that the neighboring lamella in the same bundle, share the same orientation of c-axis; and the interface between the neighboring nanolamella are perpendicular to the c-axis of $\mathrm{Ca}_{3} \mathrm{Co}_{4} \mathrm{O}_{9}$ phase.

The present study clearly shows that $\mathrm{Ca}_{3} \mathrm{Co}_{4} \mathrm{O}_{9}$ synthesized using a sol-gel chemical method was with nano-lamella structure. The nano-lamella $\mathrm{Ca}_{3} \mathrm{Co}_{4} \mathrm{O}_{9}$ within one bundle possesses the fiber texture and share the same $c$-directions. Since the nano-lamellas are with the length of a few microns and the thickness of 5-50 nm, the poly-crystals with nano-lamella are expected to have significant high density of grain boundaries existing in between the nano-lamellas. Because of the layered structure $\mathrm{Ca}_{3} \mathrm{Co}_{4} \mathrm{O}_{9}$ and strong electrical conductivity anisotropy, the polycrystal samples with nano-lamella may exhibit different electrical transport properties in comparison with those from the single crystals. So far, it is unclear about the effect of grain boundaries in between the nano-lamellas on the electrical properties. In addition, the grain boundaries may act as active scattering centers for scattering phonons and further reduce the thermo-conductivity. The present study clearly shows that the crystal texture and the nano-lamellas have significant impact on the thermal conductivity. It was evident that the baseline pellet prepared by coldpressing gives a certain degree of crystal texture. As a consequence, the thermal conductivity measured parallel to the pressed plane is $\sim 30 \%$ higher than the value measured perpendicular to the press plane, as shown in Fig 5.2. 

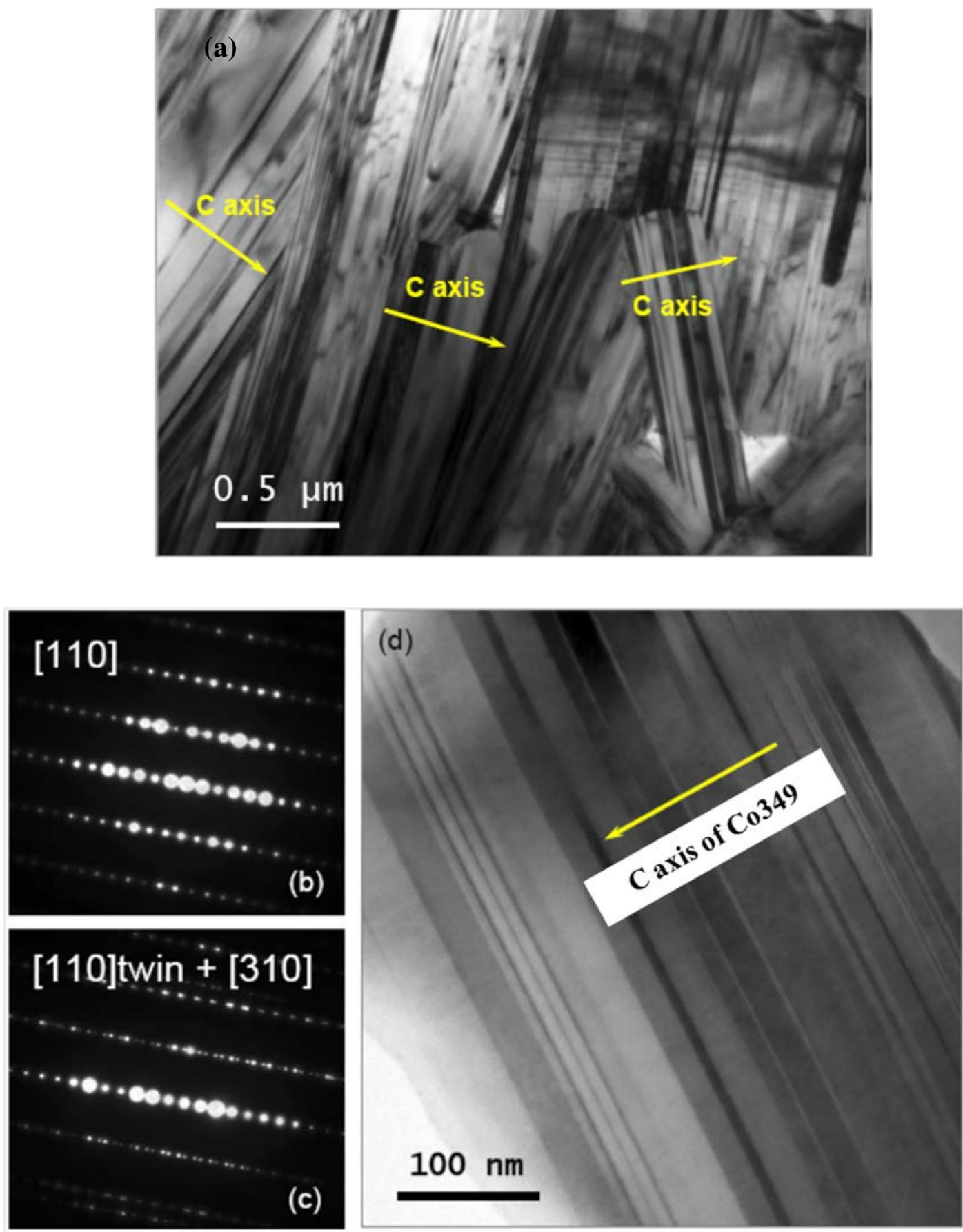

Figure 5. 6a Baseline sample: Crystal bundles with different orientation. b-d Baseline sample: Electron diffraction taken from different nano-lamella (b,c) and the magnified view of nano-lamella within one bundle. 
(2). Annealed sample. Fig 5.8 of TEM images shows that, in the annealed sample, the elongated $\mathrm{Ca}_{3} \mathrm{Co}_{4} \mathrm{O}_{9}$ bundles were with length of $1 \mu \mathrm{m}$. The nano-lamellas of the $\mathrm{Ca}_{3} \mathrm{Co}_{4} \mathrm{O}_{9}$ grains are with c-axis fiber texture. The electron diffraction in Fig 5.8 (b), that was taken from the region circled in Fig 5.8 (a), shows that some of the c-axis fiber texture deteriorate in the annealed sample and the c-axis of different lamella's deviate about less than $4^{\circ}$ in between the neighboring lamella within one bundle. In addition to the $\mathrm{Ca}_{3} \mathrm{Co}_{4} \mathrm{O}_{9}$ nano-lamellas, one of the main features observed in the annealed sample is the secondary phases that exist in between the neighboring $\mathrm{Ca}_{3} \mathrm{Co}_{4} \mathrm{O}_{9}$ nano-lamellas. The morphologies of the $\mathrm{Ca}_{3} \mathrm{Co}_{4} \mathrm{O}_{9}$ phase and the secondary phases are shown in Fig 5.9 (a). Most of the secondary phases are 5-10 nm in width and several hundred nanometers in length. The EDS results in Table 1 indicate the secondary phase contains Ca, Co and O. The complex electron diffraction pattern in Fig 5.9 (b) is taken from the region with both $\mathrm{Ca}_{3} \mathrm{Co}_{4} \mathrm{O}_{9}$ and secondary phase shown in Fig 5.9 (a). The secondary phase of Ca-Co-O phase could be indexed as $\mathrm{CaO}$ phase with the fcc structure and lattice parameter of $4.81 \AA$ (JCPDS Card No. 37-1497). The CaO phase also keep well-defined crystal orientation to the $\mathrm{Ca}_{3} \mathrm{Co}_{4} \mathrm{O}_{9}$ and is with $[110]_{\mathrm{CaO}} / /[010]_{\mathrm{C} 3349}$.

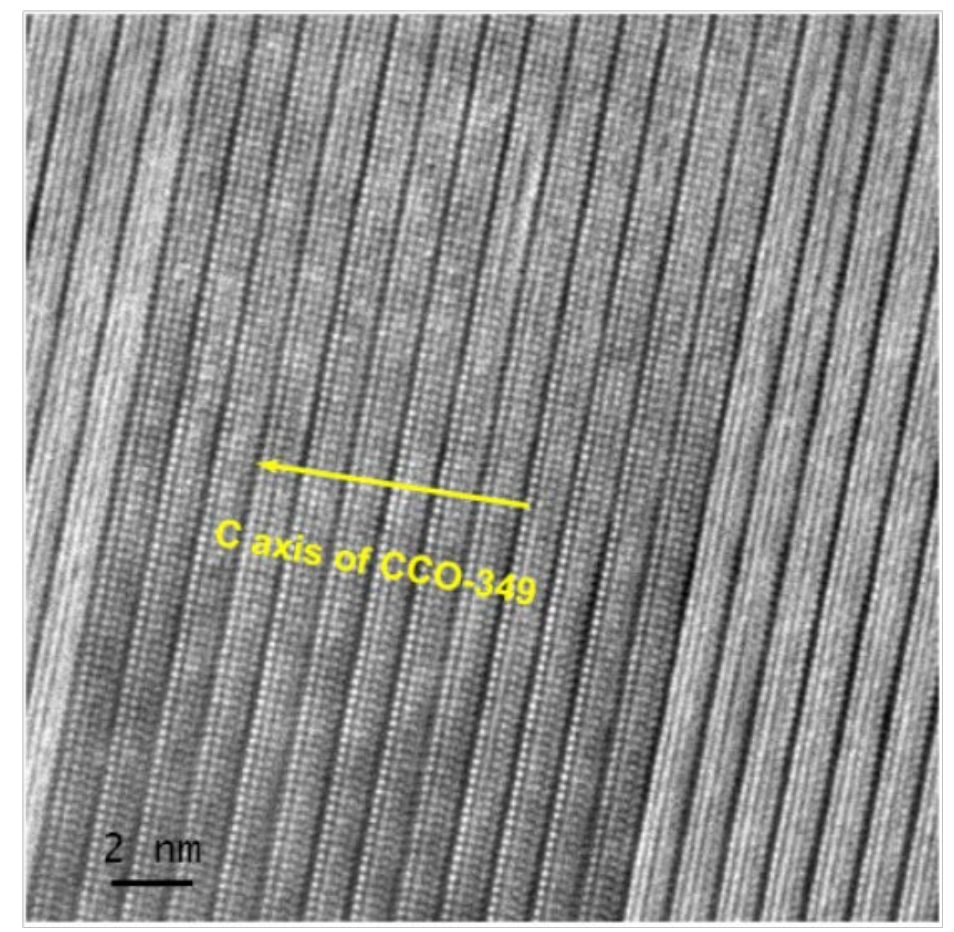

Figure 5. 7 Baseline sample: HRTEM images showing the nano-lamella with the same orientation along the $c$ axis and different orientation along the $a b$ plane. 

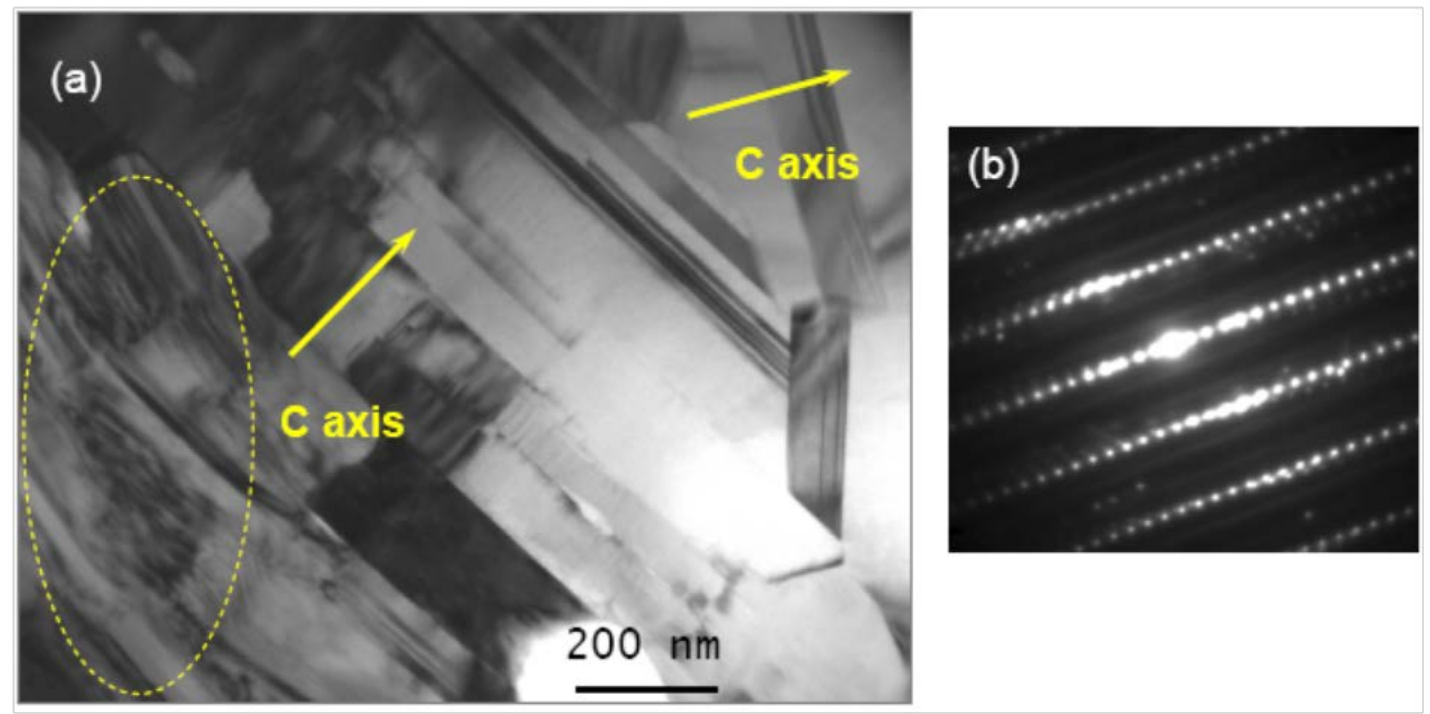

Figure 5. 8 Annealed sample: Nano-lamella Co349 and their electron diffraction pattern.

In addition to the $\mathrm{CaO}$ phase, very small amount of a Ca-Co-O cobaltite phase with nominal composition of Ca:Co:O=2:2:5 $\left(\mathrm{Ca}_{2} \mathrm{Co}_{2} \mathrm{O}_{5}\right)$ was also found. The morphology of the $\mathrm{Ca}_{2} \mathrm{Co}_{2} \mathrm{O}_{5}$ grains and EDS results are given in Fig 5.10 and Table 2, respectively. Fig 5.10 (a) and (b) clearly show that the $\mathrm{Ca}_{2} \mathrm{Co}_{2} \mathrm{O}_{5}$ grains are irregularly shaped with the grain size of several hundred nanometers. It is worthwhile to point out that, in stark contrast to the $\mathrm{Ca}_{3} \mathrm{Co}_{4} \mathrm{O}_{9}$, the $\mathrm{Ca}_{2} \mathrm{Co}_{2} \mathrm{O}_{5}$ grain contains very few defects such as nano-lamella, twinning and dislocations. To understand the crystal structure of the $\mathrm{Ca}_{2} \mathrm{Co}_{2} \mathrm{O}_{5}$ grain, the electron diffraction patterns are taken from the $\mathrm{Ca}_{2} \mathrm{Co}_{2} \mathrm{O}_{5}$ grain, and the interface region between $\mathrm{Ca}_{3} \mathrm{Co}_{4} \mathrm{O}_{9}$ and $\mathrm{Ca}_{2} \mathrm{Co}_{2} \mathrm{O}_{5}$, as shown Fig 5.11 (a) and (b), respectively. According to the reported crystallography data for the $\mathrm{Ca}_{2} \mathrm{Co}_{2} \mathrm{O}_{5}$ phase, ${ }^{[47]}$ with the pervoskite of brownmillerite type and lattice parameter of $a \approx$ $a_{p} \sqrt{2} \approx 5.46 \AA \hat{,}, b \approx 4 a_{p} \approx 14.88 \AA$ and $c \approx a_{p} \sqrt{2} \approx 5.46 \AA$, the electron diffraction in Fig 5.10 (a) is tentatively indexed as from the [010] direction of $\mathrm{Ca}_{2} \mathrm{Co}_{2} \mathrm{O}_{5}$. This index need to be further verified by systematic tilting the $\mathrm{Ca}_{2} \mathrm{Co}_{2} \mathrm{O}_{5}$ grains and recording the patterns from different zone axis. This part of the indexing for $\mathrm{Ca}_{2} \mathrm{Co}_{2} \mathrm{O}_{5}$ grain is currently ongoing and will be reported later. The electron diffraction pattern (in Fig 5.11(b)) taken from the interface of the $\mathrm{Ca}_{3} \mathrm{Co}_{4} \mathrm{O}_{9}$ and the $\mathrm{Ca}_{2} \mathrm{Co}_{2} \mathrm{O}_{5}$ also shows the $\mathrm{Ca}_{2} \mathrm{Co}_{2} \mathrm{O}_{5}$ grain possesses well defined crystallographic orientation relationship with the substrate $\mathrm{Ca}_{3} \mathrm{Co}_{4} \mathrm{O}_{9}$ phase (showing the indexed $\left.[010]_{\mathrm{Ca} 3 \mathrm{Co} 4 \mathrm{O} 9} / /[110]_{\mathrm{Ca} 2 \mathrm{Co} 2 \mathrm{O} 5}\right)$. This orientation relationship is clearly supported by the HRTEM images that taken from the interface of $\mathrm{Ca}_{2} \mathrm{Co}_{2} \mathrm{O}_{5}$ and $\mathrm{Ca}_{3} \mathrm{Co}_{4} \mathrm{O}_{9}$ phase shown in Fig. 12. 

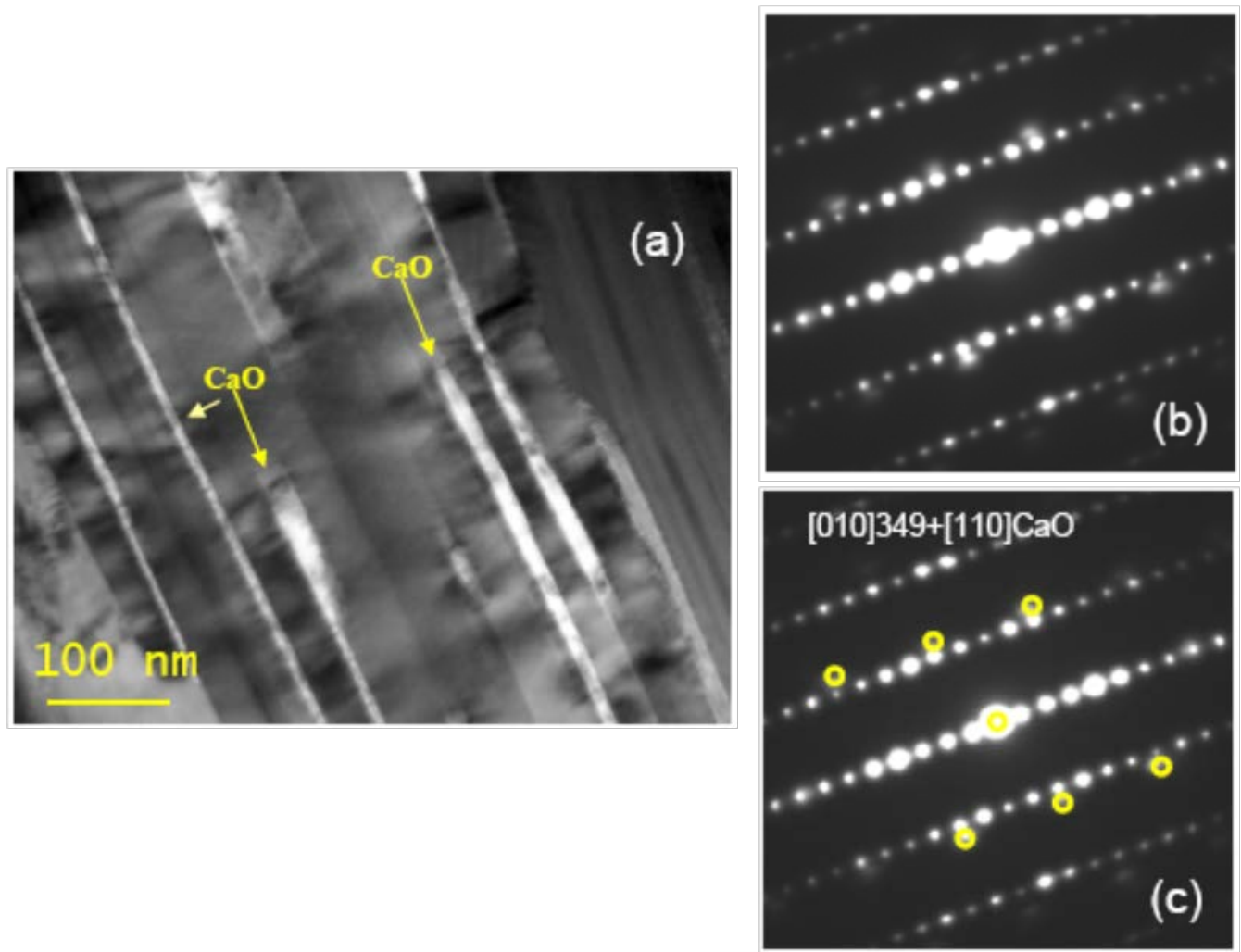

Figure 5. 9 Annealed sample: Co-containing $\mathrm{CaO}$ in between the Co349 nano-lamella. (c) is indexing for (b).
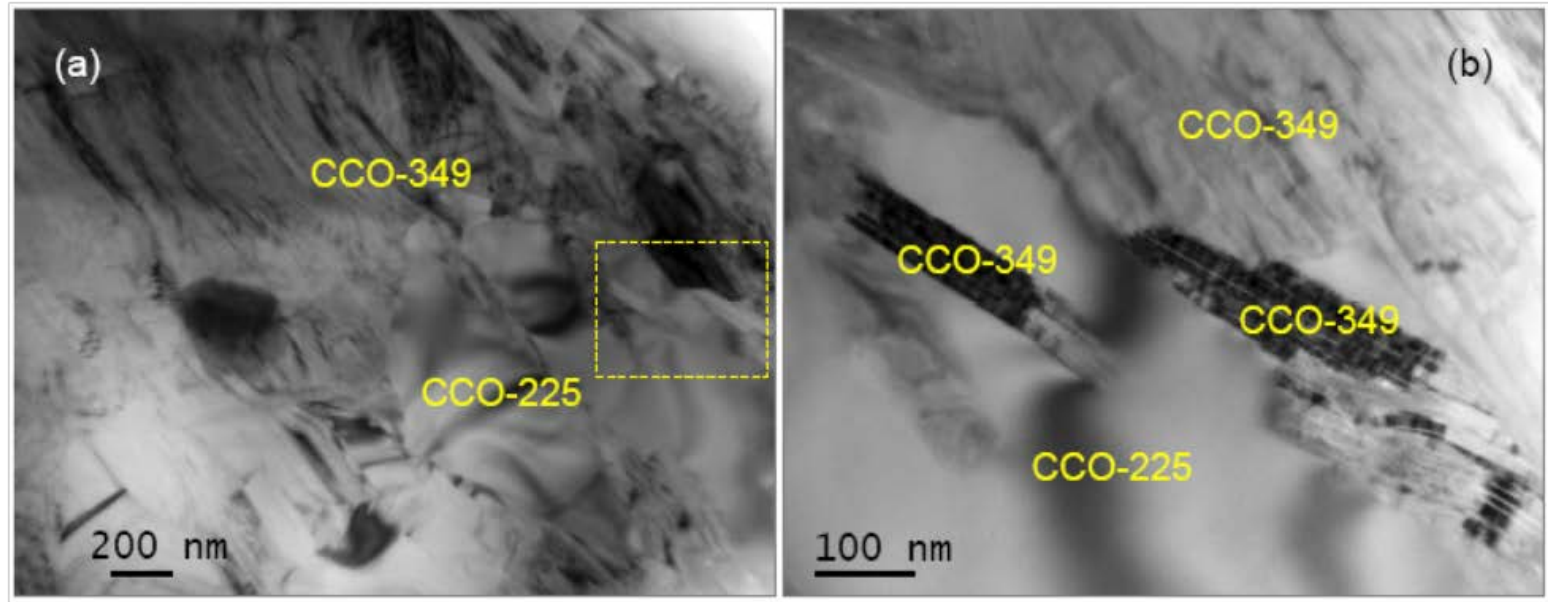

Figure 5. 10 Annealed sample: Nano-lamella Co349 (CCO349) and Co225 (CCO225). 
Table 5. 1 EDS result of $\mathrm{CaO}$ from annealed sample

\begin{tabular}{|c|c|c|c|}
\hline Element & O & Ca & Co \\
\hline Atom\% & 42.73 & 42.42 & 14.85 \\
\hline
\end{tabular}

Table 5. 2 EDS of Co225

\begin{tabular}{|c|c|c|c|}
\hline Element & O & Ca & Co \\
\hline Atom\% & 53.23 & 24.56 & 22.21 \\
\hline
\end{tabular}

\subsubsection{Thermal stability of $\mathrm{Ca}_{3} \mathrm{Co}_{4} \mathrm{O}_{9}$ upon high temperature annealing}
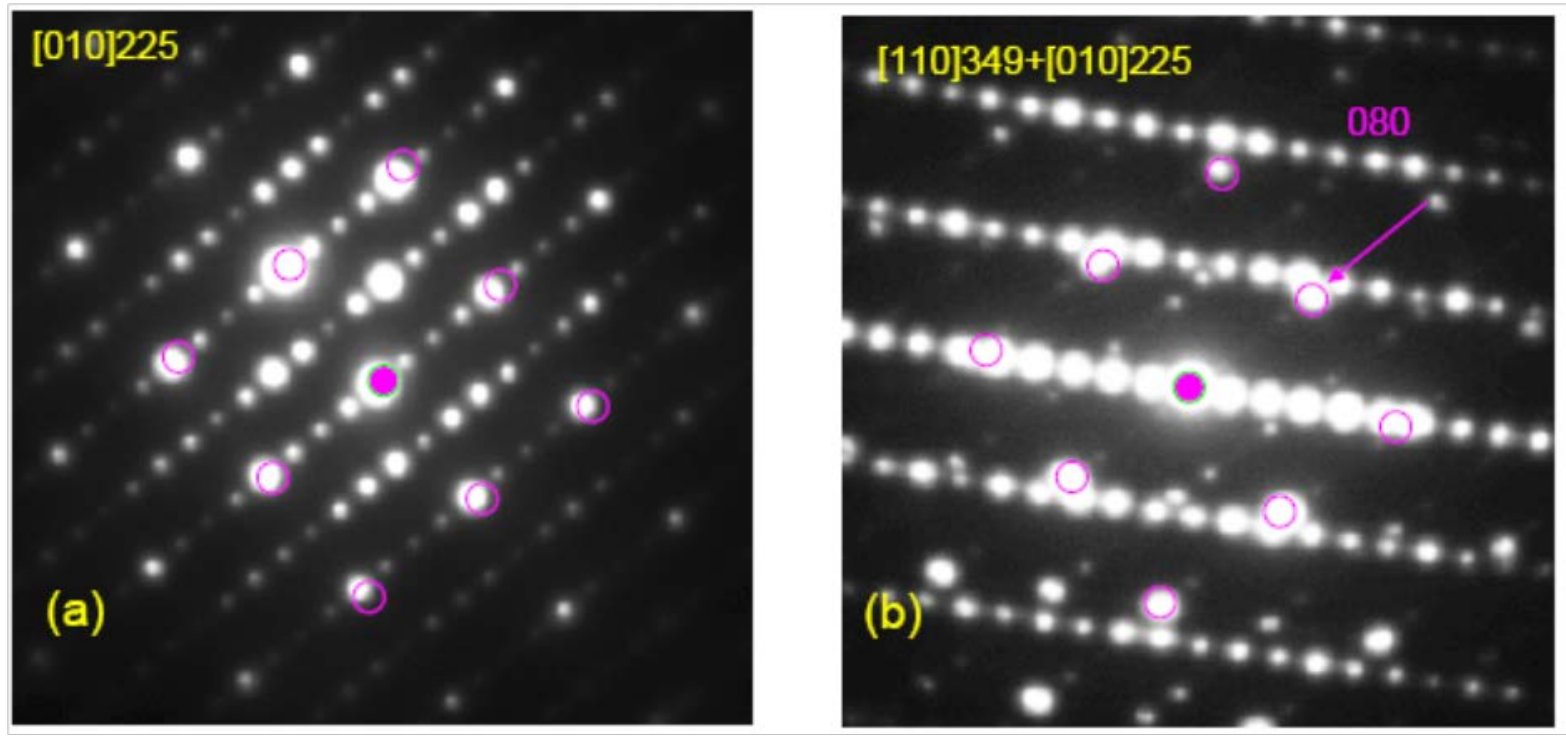

Figure 5. 11 Annealed sample: Diffraction pattern from Co225 (a) and the interface between Co225 and Co349 (b).

The present study demonstrates that the $\mathrm{Ca}_{3} \mathrm{Co}_{4} \mathrm{O}_{9}$ phase was stabilized after annealing at $1000^{\circ} \mathrm{C}$. The Seebeck coefficient decrease slightly after annealing. In the meanwhile, the annealed sample presents the increased electrical resistivity. The increased resistivity could be correlated with the nano-structure changes in the annealed sample. As evidence by the SEM image, the $\mathrm{Ca}_{3} \mathrm{Co}_{4} \mathrm{O}_{9}$ grain experienced some grain orientation changes. Because of the large anisotropy of the electron resistivity, the c-axis oriented grains on the press-plane will increase the resistivity. The electrical resistivity can be further increased due to the existence of insulating $\mathrm{CaO}$ phase. Upon high temperature annealing, the thermal conductivity anisotropy 
was eliminated. This is possibly caused by the grain orientation changes caused by the annealing, as evidenced by the SEM images. On the other hand, the $\mathrm{CaO}$ phase existing in between $\mathrm{Ca}_{3} \mathrm{Co}_{4} \mathrm{O}_{9}$ may lower the thermal diffusivity and decrease the anisotropy as well.

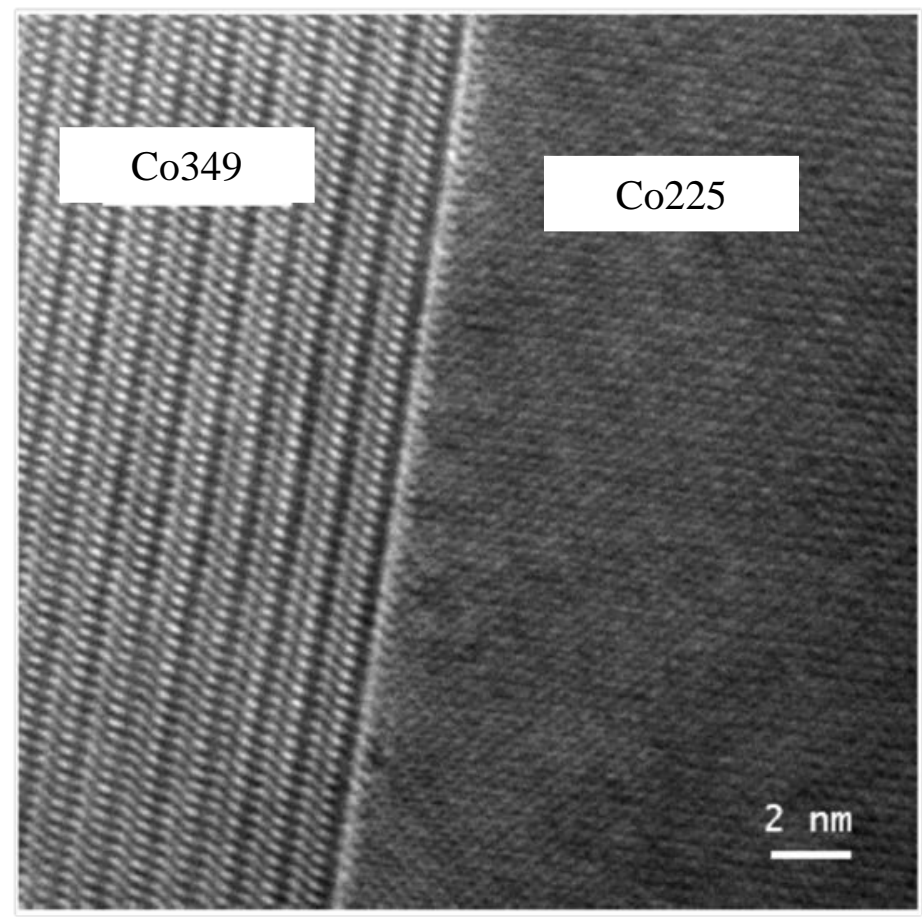

Figure 5. 12 Annealed sample: Nano-lamella Co349 and Co225.

The current study also reveals the existence of $\mathrm{Ca}_{2} \mathrm{Co}_{2} \mathrm{O}_{5}$ grains in the annealed sample. In addition to the $\mathrm{Ca}_{2} \mathrm{Co}_{2} \mathrm{O}_{5}$ grain, there is no other $\mathrm{Ca}-\mathrm{Co}-\mathrm{O}$ phase such as $\mathrm{Ca}_{3} \mathrm{Co}_{2} \mathrm{O}_{6}$ phase was found in the present study. It is noticed that compounds with a ratio $\mathrm{Ca}: \mathrm{Co}=1: 1$ can be synthesized only by special processes such as spark plasma sintering ${ }^{[22]}$ and pulsed laser deposition. ${ }^{[47]}$ To our knowledge, this is the first time that $\mathrm{Ca}_{2} \mathrm{Co}_{2} \mathrm{O}_{5}$ was found to co-exist with the $\mathrm{Ca}_{3} \mathrm{Co}_{4} \mathrm{O}_{9}$ phase in the bulk scale samples. This study will be helpful for understanding the phase stability of the complicated Ca-Co-O phase system with layered structure, and further exploring the new variants of Ca-Co-O phases such as $\mathrm{Ca}_{2} \mathrm{Co}_{2} \mathrm{O}_{5}$ and their thermoelectric properties.

\subsection{Conclusions}

Polycrystal $\mathrm{Ca}_{3} \mathrm{Co}_{4} \mathrm{O}_{9}$ bulk sample with the power factor of $310 \mu \mathrm{Wm}^{-1} \mathrm{~K}^{-2}$ at $713^{\circ} \mathrm{C}$, was synthesized using the chemical sol-gel solute. The large $\mathrm{Ca}_{3} \mathrm{Co}_{4} \mathrm{O}_{9}$ grains are composed of the nano-lamellas of $\mathrm{Ca}_{3} \mathrm{Co}_{4} \mathrm{O}_{9}$ with strong c-axis fiber texture. In addition to the large electrical properties anisotropy, the thermal conductivity measured from different directions on the baseline pellets show significant difference as well. Upon high temperature annealing at $1000^{\circ} \mathrm{C}$, 
the polycrystal $\mathrm{Ca}_{3} \mathrm{Co}_{4} \mathrm{O}_{9}$ retained the $Z T$ value of 0.13 at $750^{\circ} \mathrm{C}$. The annealed sample is with the comparable Seebeck coefficient and specific heat from room temperature up to $750^{\circ} \mathrm{C}$, and increased resistivity. The polycrystal $\mathrm{Ca}_{3} \mathrm{Co}_{4} \mathrm{O}_{9}$ pellet shows excellent thermal stability and very promising for high temperature power generation. After annealing at $1000^{\circ} \mathrm{C}$, the $\mathrm{Ca}_{3} \mathrm{Co}_{4} \mathrm{O}_{9}$ was pertained as the dominant phase with Co-containing $\mathrm{CaO}$ phase existing in between the $\mathrm{Ca}_{3} \mathrm{Co}_{4} \mathrm{O}_{9}$ lamellas, and irregular shaped $\mathrm{Ca}_{2} \mathrm{Co}_{2} \mathrm{O}_{5}$ grains with few crystal defects. Both the $\mathrm{CaO}$ phase and $\mathrm{Ca}_{2} \mathrm{Co}_{2} \mathrm{O}_{5}$ grains exhibit well-defined crystallography relationship with the $\mathrm{Ca}_{3} \mathrm{Co}_{4} \mathrm{O}_{9}$. 


\section{Chapter 6: Conclusions and Future Work}

\subsection{Conclusions}

In this work, the dependence of thermoelectric properties of $\mathrm{Ca}_{3} \mathrm{Co}_{4} \mathrm{O}_{9}$ on calcinations condition of powder precursor and on pelletizing pressure of bulk ceramic, and the thermal stability of $\mathrm{Ca}_{3} \mathrm{Co}_{4} \mathrm{O}_{9}$ have been investigated.

The calcinations temperature of powder precursor has a critical effect on the thermoelectric properties of bulk ceramic. Four different temperatures, $650^{\circ} \mathrm{C}, 700^{\circ} \mathrm{C}, 750^{\circ} \mathrm{C}$, and $800^{\circ} \mathrm{C}$, were used for powder calcinations. The overall electrical conductivity and the power factor along the pressed plane are increased with decreasing the calcinations temperature for powder. Powders calcination at $650^{\circ} \mathrm{C}, 700^{\circ} \mathrm{C}$ results in the pellet power factor peaking at $300 \mathrm{mw} / \mathrm{mK}^{2}$, which is 1.25 times of that from the pellets with the powders calcined at $800^{\circ} \mathrm{C}$. In the meanwhile, thermal conductivity along out-of-plane direction was increased with increasing the calcinations temperature. Powders calcinination at $650^{\circ} \mathrm{C}, 700^{\circ} \mathrm{C}$ results in the low thermal conductivty of $70 \%$ of that from the pellets with the powders calcined at $800^{\circ} \mathrm{C}$. The highest electrical conductivity and lowest thermal-conductivity of the pellets were with uniform grain size distribution. By contrast, the pellets made from powders calcined at $750-800^{\circ} \mathrm{C}$ exhibit bi-modal grain distribution.

The dependence of thermoelectric properties of $\mathrm{Ca}_{3} \mathrm{Co}_{4} \mathrm{O}_{9}$ on the pelletizing pressure was obtained. Lotergering factors calculated from XRD results have proved that the texturing extent in our samples was not as large as that in those samples fabricated by special methods. Although increasing the pelletizing pressure, the electrical resistivity was increased along in-plane direction, but the figure-of-merit was not improved since the thermal conductivity was also increased along the same direction.

The thermal stability of $\mathrm{Ca}_{3} \mathrm{Co}_{4} \mathrm{O}_{9}$ bulk sample was investigated. The polycrystal $\mathrm{Ca}_{3} \mathrm{Co}_{4} \mathrm{O}_{9}$ pellet shows excellent thermal stability and very promising for high temperature power generation. Upon high temperature annealing at $1000^{\circ} \mathrm{C}$, the polycrystal $\mathrm{Ca}_{3} \mathrm{Co}_{4} \mathrm{O}_{9}$ retained the $Z T$ value of 0.13 at $750^{\circ} \mathrm{C}$. The annealed sample is with the comparable Seebeck coefficient and specific heat from room temperature up to $750^{\circ} \mathrm{C}$, and increased resistivity. After annealing at $1000^{\circ} \mathrm{C}$, the $\mathrm{Ca}_{3} \mathrm{Co}_{4} \mathrm{O}_{9}$ was pertained as the dominant phase with Co-containing $\mathrm{CaO}$ phase existing in between the $\mathrm{Ca}_{3} \mathrm{Co}_{4} \mathrm{O}_{9}$ lamellas, and irregular shaped $\mathrm{Ca}_{2} \mathrm{Co}_{2} \mathrm{O}_{5}$ grains with few crystal defects. Both the $\mathrm{CaO}$ phase and $\mathrm{Ca}_{2} \mathrm{Co}_{2} \mathrm{O}_{5}$ grains exhibit well-defined crystallography relationship with the $\mathrm{Ca}_{3} \mathrm{Co}_{4} \mathrm{O}_{9}$. The large $\mathrm{Ca}_{3} \mathrm{Co}_{4} \mathrm{O}_{9}$ grains are composed of the nano-lamellas of $\mathrm{Ca}_{3} \mathrm{Co}_{4} \mathrm{O}_{9}$ with strong c-axis fiber texture.

\subsection{Future Work}

Enhancing the TE performance of $\mathrm{Ca}_{3} \mathrm{Co}_{4} \mathrm{O}_{9}$ through doping transition metals and/or rareearth metals at Co site and Ca site could improve the TE performance of $\mathrm{Ca}_{3} \mathrm{Co}_{4} \mathrm{O}_{9}$. It is stated 
that, by appropriate doping of trivalent heavy Lanthanide elements for $\mathrm{Ca}^{2+}$ in the $\mathrm{Ca}_{3} \mathrm{Co}_{4} \mathrm{O}_{9}$ system, an increase of Seebeck coefficient could be achieved. Upon the completion of the microstructure and properties optimization of polycrystal pure $\mathrm{Ca}_{3} \mathrm{Co}_{4} \mathrm{O}_{9}$, investigation will be carried out to further improve the thermoelectric performance of $\mathrm{Ca}_{3} \mathrm{Co}_{4} \mathrm{O}_{9}$ through doping impurity and engineering the nanostructure. 


\section{References}

1. Terasaki, I.; Sasago, Y.; Uchinokura, K., Large thermoelectric power in $\mathrm{NaCo}_{2} \mathrm{O}_{4}$ single crystals. Phys. Rev. B 1997, 56 (20), 12685-12687.

2. Shikano, M.; Funahashi, R., Electrical and thermal properties of single-crystalline $\left(\mathrm{Ca}_{2} \mathrm{CoO}_{3}\right)_{0.7} \mathrm{CoO}_{2}$ with a $\mathrm{Ca}_{3} \mathrm{Co}_{4} \mathrm{O}_{9}$ structure. Appl. Phys. Lett. 2003, 82 (12), 1851-1853.

3. Katsuyama, S.; Takiguchi, Y.; Ito, M., Synthesis of $\mathrm{Ca}_{3} \mathrm{Co}_{4} \mathrm{O}_{9}$ ceramics by polymerized complex and hydrothermal hot-pressing processes and the investigation of its thermoelectric properties. J. Mater. Sci. 2008, 43 (10), 3553-3559.

4. Wang, Y.; Sui, Y.; Wang, X. J.; Su, W. H.; Liu, X. Y., Enhanced high temperature thermoelectric characteristics of transition metals doped $\mathrm{Ca}_{3} \mathrm{Co}_{4} \mathrm{O}_{9+\delta}$ by cold high-pressure fabrication. J. Appl. Phys. 2010, 107 (3), 033708.

5. Zhou, Y. Q.; Matsubara, I.; Horii, S.; Takeuchi, T.; Funahashi, R.; Shikano, M.; Shimoyama, J.; Kishio, K.; Shin, W.; Izu, N.; Murayama, N., Thermoelectric properties of highly grain-aligned and densified Co-based oxide ceramics. J. Appl. Phys. 2003, 93 (5), 2653-2658.

6. Zhang, Y. F.; Zhang, J. X.; Lu, Q. M., Synthesis of highly textured $\mathrm{Ca}_{3} \mathrm{CO}_{4} \mathrm{O}_{9}$ ceramics by spark plasma sintering. Ceram. Int. 2007, 33 (7), 1305-1308.

7. Kenfaui, D.; Bonnefont, G.; Chateigner, D.; Fantozzi, G.; Gomina, M.; Noudem, J. G., $\mathrm{Ca}_{3} \mathrm{Co}_{4} \mathrm{O}_{9}$ ceramics consolidated by SPS process: Optimisation of mechanical and thermoelectric properties. Mater. Res. Bull. 2010, 45 (9), 1240-1249.

8. Mahan, G. D., Good thermoelectrics. In Solid State Physics, Vol 51: Advances in Research and Applications, Ehrenreich, H.; Spaepen, F., Eds. Elsevier Academic Press Inc: San Diego, 1998; Vol. 51, pp 81-157.

9. Morelli, D. T., Encyclopedia of Applied Physics. 1997; Vol. 21, p 339.

10. Li, J. F.; Liu, W. S.; Zhao, L. D.; Zhou, M., High-performance nanostructured thermoelectric materials. Npg Asia Materials 2010, 2 (4), 152-158.

11. Tritt, T. M.; Subramanian, M. A., Thermoelectric materials, phenomena, and applications: a bird's eye view. MRS BULLETIN 2006, 31, 188-194.

12. Koumoto, K.; Terasaki, I.; Funahashi, R., Complex oxide materials for potential thermoelectric applications. MRS BULLETIN 2006, 31 (3), 206-210.

13. Masuda, Y.; Nagahama, D.; Itahara, H.; Tani, T.; Seo, W. S.; Koumoto, K., Thermoelectric performance of Bi- and Na-substituted $\mathrm{Ca}_{3} \mathrm{Co}_{4} \mathrm{O}_{9}$ improved through ceramic texturing. J. Mater. Chem. 2003, 13 (5), 1094-1099.

14. Moon, J. W.; Nagahama, D.; Masuda, Y.; Seo, W. S.; Koumoto, K., Anisotropic thermoelectric properties of crystal-axis oriented ceramics of layer-structured oxide in the Ca-Co-O system. J. Ceram. Soc. Jpn. 2001, 109 (8), 647-650.

15. Fergus, J. W., Oxide materials for high temperature thermoelectric energy conversion. J. European Ceram. Soc. 2012, 32 (3), 525-540.

16. Kenfaui, D.; Chateigner, D.; Gomina, M.; Noudem, J. G., Texture, mechanical and thermoelectric properties of $\mathrm{Ca}_{3} \mathrm{Co}_{4} \mathrm{O}_{9}$ ceramics. J. Alloy. Compd. 2010, 490 (1-2), 472-479.

17. Tai, L. W.; Lessing, P. A., Modifies resin intermediate processing of perovskite powders. 2. processing for fine, nonagglomerated Sr-doped Lanthanum Chromite powders. J. Mater. Res. 1992, 7 (2), 511-519.

18. Tang, G. D.; Guo, H. H.; Yang, T.; Zhang, D. W.; Xu, X. N.; Wang, L. Y.; Wang, Z. H.; Wen, H. H.; Zhang, Z. D.; Du, Y. W., Anisotropic thermopower and magnetothermopower in a misfit-layered calcium cobaltite. Appl. Phys. Lett. 2011, 98 (20).

19. Woermann, E.; Muan, A., PHASE EQUILIBRIA IN SYSTEM CAO-COBALT OXIDE IN AIR. Journal of Inorganic \& Nuclear Chemistry 1970, 32 (5), 1455-\&. 
20. Tahashi, M.; Tanimoto, T.; Goto, H.; Takahashi, M.; Ido, T., Sintering Temperature Dependence of Thermoelectric Performance and Crystal Phase of Calcium Cobalt Oxides. J. Am. Ceram. Soc. 2010, 93 (10), 3046-3048.

21. Vidyasagar, K.; Gopalakrishnan, J.; Rao, C. N. R., A convenient route for the synthesis of complex metal-oxides employing solid-solution precursors. Inorganic Chemistry 1984, 23 (9), 1206-1210.

22. Zhang, Y. F.; Zhang, J. X.; Lu, Q. M., Rapid synthesis of Ca2Co2O5 textured ceramics by coprecipitation method and spark plasma sintering. Journal of Alloys and Compounds 2005, 399 (1-2), 64-68.

23. Lan, J. L.; Lin, Y. H.; Li, G. J.; Xu, S. L.; Liu, Y.; Nan, C. W.; Zhao, S. J., High-temperature electrical transport behaviors of the layered $\mathrm{Ca}_{2} \mathrm{Co}_{2} \mathrm{O}_{5}$-based ceramics. Applied Physics Letters 2010, 96 (19), 192104.

24. Masset, A. C.; Michel, C.; Maignan, A.; Hervieu, M.; Toulemonde, O.; Studer, F.; Raveau, B.; Hejtmanek, J., Misfit-layered cobaltite with an anisotropic giant magnetoresistance: $\mathrm{Ca}_{3} \mathrm{Co}_{4} \mathrm{O}_{9}$. Phys. Rev . B 2000, 62 (1), 166-175.

25. Boullay, P.; Dorcet, V.; Perez, O.; Grygiel, C.; Prellier, W.; Mercey, B.; Hervieu, M., Structure determination of a brownmillerite $\mathrm{Ca}(2) \mathrm{Co}(2) \mathrm{O}(5)$ thin film by precession electron diffraction. Physical Review B 2009, 79 (18).

26. Fjellvag, H.; Gulbrandsen, E.; Aasland, S.; Olsen, A.; Hauback, B. C., Crystal structure and possible charge ordering in one-dimensional $\mathrm{Ca}_{3} \mathrm{Co}_{2} \mathrm{O}_{6}$. J. Solid State Chem. 1996, 124 (1), 190-194.

27. Maignan, A.; Michel, C.; Masset, A. C.; Martin, C.; Raveau, B., Single crystal study of the one dimensional Ca3Co2O6 compound: five stable configurations for the Ising triangular lattice. European Physical Journal B 2000, 15 (4), 657-663.

28. Kageyama, H.; Yoshimura, K.; Kosuge, K.; Mitamura, H.; Goto, T., Field-induced magnetic transitions in the one-dimensional compound Ca3Co2O6. Journal of the Physical Society of Japan 1997, 66 (6), 1607-1610.

29. Kageyama, H.; Yoshimura, K.; Kosuge, K.; Azuma, M.; Takano, M.; Mitamura, H.; Goto, T., Magnetic anisotropy of Ca3Co2O6 with ferromagnetic Ising chains. Journal of the Physical Society of Japan 1997, 66 (12), 3996-4000.

30. Pechini, M. P. U.S. Pat. NO.3 330 697, July 11, 1967.

31. Tai, L. W.; Lessing, P. A., Modifies resin intermediate processing of perovskite powders. 1. optimization of polymeric precursors. J. Mater. Res. 1992, 7 (2), 502-510.

32. Thompson, C. V., Secondary grain-growth in thin-films of semiconductors - Theorectical aspects. $J$. Appl. Phys. 1985, 58 (2), 763-772.

33. Lotgering, F. K., Topotactical reactions with ferrimagnetic oxides having hexagonal crystal strutures. . Journal of Inorganic \& Nuclear Chemistry 1959, 9 (2), 113-\&.

34. Takeuchi, T.; Tani, T.; Saito, Y., Piezoelectric properties of bismuth layer-structured ferroelectric ceramics with a preferred orientation processed by the reactive templated grain growth method. Jpn. $J$. Appl. Phys. Part 1 - Regul. Pap. Short Notes Rev. Pap. 1999, 38 (9B), 5553-5556.

35. JCPDS Card No. 21-139.

36. Tsai, P. H.; Li, S.; Tay, Y. Y.; Chen, Z. D., Texturing behaviors and kinetics of $\mathrm{NaCo}_{2} \mathrm{O}_{4-\delta}$ thermoelectric materials. J. Am. Ceram. Soc. 2007, 90 (6), 1908-1911.

37. Takada, K.; Sakurai, H.; Takayama-Muromachi, E.; Izumi, F.; Dilanian, R. A.; Sasaki, T., Superconductivity in two-dimensional $\mathrm{CoO}_{2}$ layers. Nature 2003, 422 (6927), 53-55.

38. Singh, D. J., Quantum critical behavior and possible triplet superconductivity in electron-doped CoO2 sheets. Phys. Rev. B 2003, 68 (2).

39. Shizuya, M.; Isobe, M.; Baba, Y.; Nagai, T.; Osada, M.; Kosuda, K.; Takenouchi, S.; Matsui, Y.; Takayama-Muromachi, E., New misfit-layered cobalt oxide $(\mathrm{CaOH})_{1.14} \mathrm{CoO}_{2}$. J. Solid State Chem. 2007, 180 (1), 249-259. 
40. Isobe, M.; Shizuya, M.; Takayama-Muromachi, E., Crystal structure and physical properties of a misfit-layered cobaltite $(\mathrm{CaOH})(1.14) \mathrm{CoO} 2$. Journal of Magnetism and Magnetic Materials 2007, 310 (2), E269-E271.

41. Miyazaki, Y.; Onoda, M.; Oku, T.; Kikuchi, M.; Ishii, Y.; Ono, Y.; Morii, Y.; Kajitani, T., Modulated structure of the thermoelectric compound $\left[\mathrm{Ca}_{2} \mathrm{CoO}_{3}\right]_{0.62} \mathrm{CoO}_{2}$. J. Phys. Soc. Jpn. 2002, 71 (2), 491-497.

42. De Vaulx, C.; Julien, M. H.; Berthier, C.; Hebert, S.; Pralong, V.; Maignan, A., Electronic correlations in $\mathrm{CoO} 2$, the parent compound of triangular cobaltates. Phys. Rev. Lett. 2007, 98 (24).

43. Matsubara, I.; Funahashi, R.; Shikano, M.; Sasaki, K.; Enomoto, H., Cation substituted $\left[\mathrm{Ca}_{2} \mathrm{CoO}_{3}\right]_{x} \mathrm{CoO}_{2}$ films and their thermoelectric properties. Appl. Phys. Lett. 2002, 80 (25), 4729-4731.

44. Xu, G. J.; Funahashi, R.; Shikano, M.; Matsubara, I.; Zhou, Y. Q., Thermoelectric properties of the Bi- and Na- substituted $\mathrm{Ca}_{3} \mathrm{Co}_{4} \mathrm{O}_{9}$ system. Appl. Phys. Lett. 2002, 80 (20), 3760-3762.

45. Hu, Y. F.; Si, W. D.; Sutter, E.; Li, Q., In situ growth of c-axis-oriented $\mathrm{Ca}_{3} \mathrm{Co}_{4} \mathrm{O}_{9}$ thin films on Si(100). Appl. Phys. Lett. 2005, 86 (8), 082103.

46. Minnich, A. J.; Dresselhaus, M. S.; Ren, Z. F.; Chen, G., Bulk nanostructured thermoelectric materials: current research and future prospects. Energy \& Environmental Science 2009, 2 (5), 466-479.

47. Boullay, P.; Dorcet, V.; Perez, O.; Grygiel, C.; Prellier, W.; Mercey, B.; Hervieu, M., Structure determination of a brownmillerite $\mathrm{Ca}_{2} \mathrm{Co}_{2} \mathrm{O}_{5}$ thin film by precession electron diffraction. Physical Review $B$ 2009, 79 (18).

48. Pei, J.; Chen, G.; Li, X.; Li, Y. X.; Zhou, N., Molten salt synthesis and thermoelectric properties of $\mathrm{Ca}(2) \mathrm{Co}(2) \mathrm{O}(5)$. Materials Letters 2009, 63 (17), 1459-1461.

49. Cushing, B. L.; Wiley, J. B., Topotactic routes to layered calcium cobalt oxides. J. Solid State Chem. 1998, 141 (2), 385-391.

50. Anderson, M. T.; Vaughey, J. T.; Poeppelmeier, K. R., STRUCTURAL SIMILARITIES AMONG OXYGEN-DEFICIENT PEROVSKITES. Chem. Mat. 1993, 5 (2), 151-165.

51. Sharma, N.; Shaju, K. M.; Rao, G. V. S.; Chowdari, B. V. R., Mixed oxides $\mathrm{Ca}_{2} \mathrm{Fe}_{2} \mathrm{O} 5$ and $\mathrm{Ca}_{2} \mathrm{Co}_{2} \mathrm{O}_{5}$ as anode materials for Li-ion batteries. Electrochimica Acta 2004, 49 (7), 1035-1043. 